\title{
Real-time scheduling in outpatient clinics
}

Citation for published version (APA):

Munavalli, J. R. (2017). Real-time scheduling in outpatient clinics. [Doctoral Thesis, Maastricht University]. Maastricht University. https://doi.org/10.26481/dis.20171101jrm

Document status and date:

Published: 01/01/2017

DOI:

10.26481/dis.20171101jrm

Document Version:

Publisher's PDF, also known as Version of record

\section{Please check the document version of this publication:}

- A submitted manuscript is the version of the article upon submission and before peer-review. There can be important differences between the submitted version and the official published version of record.

People interested in the research are advised to contact the author for the final version of the publication, or visit the DOI to the publisher's website.

- The final author version and the galley proof are versions of the publication after peer review.

- The final published version features the final layout of the paper including the volume, issue and page numbers.

Link to publication

\footnotetext{
General rights rights.

- You may freely distribute the URL identifying the publication in the public portal. please follow below link for the End User Agreement:

www.umlib.nl/taverne-license

Take down policy

If you believe that this document breaches copyright please contact us at:

repository@maastrichtuniversity.nl

providing details and we will investigate your claim.
}

Copyright and moral rights for the publications made accessible in the public portal are retained by the authors and/or other copyright owners and it is a condition of accessing publications that users recognise and abide by the legal requirements associated with these

- Users may download and print one copy of any publication from the public portal for the purpose of private study or research.

- You may not further distribute the material or use it for any profit-making activity or commercial gain

If the publication is distributed under the terms of Article $25 \mathrm{fa}$ of the Dutch Copyright Act, indicated by the "Taverne" license above, 


\section{REAL-TIME SCHEDULING IN OUTPATIENT CLINICS}

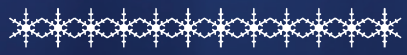

Jyoti Raghavendra Munavalli 



\section{Real-time Scheduling in Outpatient Clinics}

\section{DISSERTATION}

To obtain the degree of Doctor at the Maastricht University, on the authority of the Rector Magnificus, Prof. Dr. Rianne Letschert, in accordance with the decision of the Board of Deans, to be defended in public

on Wednesday November 1, 2017, at 12:00 hours

by

Jyoti Raghavendra Munavalli 


\section{Supervisor:}

Prof. Dr. G.G. van Merode

\section{Co-supervisor:}

Dr. Shyam Vasudeva Rao

\section{Assessment Committee:}

Prof. Dr. D. Ruwaard (Chairman)

Prof. Dr. H.J.J.M. Berden (Tilburg University)

Prof. Dr. J.F.M. Smits

Prof. Dr. J. de Vries (University of Groningen)

Prof. Dr. C.A.B. Webers

The research presented in this thesis was conducted at CAPHRI Care and Public Health Research Institute, Health Service Research Department, of Maastricht University. CAPHRI participates in the Netherlands School of Primary Care Research CaRe.

This research was financially supported by the Maastricht University Medical Centre (MUMC). Part of the research was performed in Aravind Eye Hospital, Madurai, India. 
To my mother, who has always supported me. 



\section{Contents}

1. Introduction 1

2. Background/Related Work 15

3. Study Methodology 25

4. A robust predictive resource planning under demand uncertainty to improve waiting times in outpatient clinics 35

5. Integral patient scheduling to reduce patient waiting times in outpatient clinics

6. Modelling an outpatient clinic as a Multi-Agent System for real-time coordination to improve waiting times 75

$\begin{array}{ll}\text { 7. Synthesis and Discussion } & 100\end{array}$

Valorisation

Summary

Acknowledgements

About the Author 



\section{Introduction}

The content has been published partly in:

Munavalli JR, Rao SV, Srinivasan A, Srinivas A, van Merode F. The optimization in workflow management. J Health Manage 2016;18(1):21-30. doi: 10.1177/0972063415625570. 



\section{INTRODUCTION}

In June 2011, Mary went $50 \mathrm{~km}$ from her village to an eye outpatient clinic (OPC) that gets a high volume of patients. Mary was a walk-in patient. She travelled for $1 \frac{1}{2} \mathrm{~h}$ to reach the hospital. She was aware of the long waiting time; hence she took some material for knitting during the waiting period. She was not sure of the duration of the clinic visit. When she arrived, the clinic was overcrowded with patients waiting in the queue.

Another hospital provides prior appointments to patients. Ram scheduled his appointment with the outpatient services. The doctor arrived late and then; the first two patients took a lot of the doctor's time. Ram arrived at the scheduled time but had to wait as the preceding patient's session had not yet finished.

Waiting for medical care has become the most common part of the clinic or hospital experience.

Patients usually wait for long periods before they get the required care in a hospital. Both these patients, the walk-in and the scheduled, waited. A patient arriving in pain and grief expects quality and timely care, ${ }^{[1,2]}$ but is forced to wait throughout several processes, such as registration, consultation, laboratory, billing and pharmacy. ${ }^{[3,4]}$ The prolonged waiting is unpleasant, and the patient's health is liable to deteriorate, due to the entanglement in various activities related to patient care and prolonged exposure to other patients' infections.

In both the cases as mentioned above the resources were planned and scheduled well ahead of the day. Walk-in patients like Mary cause unpredictable variations in the patients' volume and arrival time. The random arrivals of walk-in patients do not match the pace of the scheduled service of the outpatient resources, resulting in the waiting of other patients. When patient arrival is by appointment, an OPC might have a considerable amount of prior information about each patient. However, the second OPC still could not match its service pace with the patients' demand. Therefore, it is the demand that determines the target conditions for the pace of OPC service.

Waiting times have recently been attracting national and international attention and are alerting hospital managements about the redesign and improvisation of the outpatient care. Waiting times are largely the result of inadequate or untimely or wrong coordination. The OPCs, in general, are functionally organised, and the departments are managed locally and sub-optimally, and the efficiency of individual departments does not necessarily improve the overall efficiency of the OPC..$^{[5-8]}$

To date, ideas for process control optimisation and coordination have mainly come from research in industry, including queuing theory, business process reengineering, six sigma, 
lean systems, demand forecasts, staff scheduling and user scheduling. ${ }^{[5,9-11]}$ The utilisation of such measures in hospitals is effectively illustrated but is not sufficient as a solution.

The OPC with larger latency in their operations, as in the example, is a push system when the capacity of delivering care is modified on the basis of the projected average demand. However, care is provided only when the resources are ready and not when the patient needs or asks for it. ${ }^{[1]}$ A system where care is delivered when the patient asks is a pull system and is based on system status. ${ }^{[12]}$ The distinction between push and pull is to characterise logistic systems and is used in the lean management methodology (abbreviated to "lean"). Lean has become increasingly popular in industry after the publication of the book titled "the machine that changed the world" by James Womack et al. ${ }^{[13]}$ The book described the results of the MIT study on worldwide production practices in the automotive industry and showed the superiority of Japanese production systems, in particular, the "Toyota Production System" or "TPS." All major automobile brands have switched to lean or integrated it into their production system. The lean methodology has been increasingly applied to other industries, like the service industry and health care. Since major differences can be found between industries, the question is whether lean can be applied to other industries in the same way as in automobile industry and to what level the lean application needs translation and adaptation and whether it is applicable at all.

There are good reasons for being careful with using words like "lean," "push" and "pull." Most real-world systems have aspects of both push and pull. TPS implements "just-in-time" (JIT), that is, "the right thing at the right place at the right time in the right quantity" utilising both push and pull. ${ }^{[14,15]}$ TPS applies JIT for a system and not for a single department. It means that a department should not work either slower or faster than the other departments in the line. A part of the system's activity may also be a combination of push and pull. Fast-food chains store raw materials based on the projected demand (push), whereas burgers are prepared on the basis of customers' order (pull). Hence, a good operating system probably uses a mix of push and pull to achieve a continuous flow in the system. ${ }^{[16]}$ The appropriate mix depends on the demand to which the system or hospital must react to.

The patient demand is huge in developing countries like India, due to factors like ageing population and various ailments; and at the same time, there is a shortage of health care workers, which enlarges the gap between supply and demand. Such a situation results in prolonged waiting, low utilisation of resources and expensive healthcare. OPCs confront the challenge of matching their limited supply with a huge patient demand, in short time horizons, like hours and same-day. The current methods of workflow management, a function of the operating system, do not always accomplish the desired outcomes. Hence, an operating system for hospitals, which would be able to satisfy patients' demand within short cycle times at a reasonable cost, should be designed. There is a lack of knowledge in optimising OPCs on a real-time basis to improve the efficiency and flow of patients, where the actual 
or real-time demand is unknown and unstable. ${ }^{[16]}$ Therefore, this thesis aims to extend the knowledge concerning hospital operating systems by testing the hypothesis that "a hospital system with disparate subsystems cannot minimise cycle/waiting time by separately optimising subsystems, or by scheduling that does not adjust in real-time." Therefore, optimisation in real-time is a target condition which means that we have to design a system that synchronises to "keep the flow" with minimum buffers.

The scope of this thesis is limited to human resources (service delivered by doctors and other hospital staff) and patient flow. The flow of other resources through the hospital, such as capital and materials (supplies required for operations, medical documents, etc.), is not considered. First, the characteristics of OPCs are described. Next, we describe push-pull, planning and control, and connect/relate them to OPC operations systems. This chapter closes with a study aim, research questions and an outline of the thesis.

\section{OPC SYSTEM}

The OPC system is a system with interdependent and interacting components, such as an arrangement of buildings and layouts, human interactions, complex logistics, materials, technology, information and patient care. The basic elements of the OPC system are the resources like equipment, staff, procedures, information and processes, etc. (supply). The people with medical problems are referred to as patients (demand) who form the input to the OPC system.

When a patient arrives at the OPC, the appropriate processes will cure the person, halt or slow degeneration, or limit suffering. The time spent by a patient with doctors or other healthcare staff is called service time and depends on the patient's specific condition. Usually, the procedures depend on the outcomes of the previous steps, which lead to the selection of the appropriate clinical pathway for the patient. ${ }^{[17]}$ Erratic clinical pathways in the care processes require patients to move through different departments in the OPC, as the departments are functionally organised. ${ }^{[8]}$ Therefore, the patient spends more time in moving from one location to another (travel time). In order to complete the care processes, patients wait in queues in various departments. ${ }^{[18]}$ The distances between different departments affect not only the travel time of the patients but also resources, materials and costs associated with the movement. In between, the patient waits for his/her turn, due to several reasons, such as late arrival of a doctor, an early arrival of a patient, a former patient taking more time than scheduled, or (rarely) medical equipment not working. The patient has to wait in all these cases. Cycle time is the time spent by patients in the OPC, from entry to exit, constituting service time, waiting time and travel time. An OPC is an open-loop system, influenced by its environment. Outcomes like waiting time and cycle time are affected by patient volume, patient types, resources and the management and control of the OPC. 


\section{VARIABILITY AND UNCERTAINTY}

Patient arrivals at the OPC are scheduled (through appointments), or walk-in, or mixed. In most of the Indian OPCs, patient arrival is either walk-in or mixed, resulting in the unpredictability of patient volume. Even OPCs with only scheduled patients face problems, due to the early or late arrival of patients or doctors, no-show of patients and cancelled schedules. The arrival pattern of walk-in patients is not uniform throughout the day. It mostly follows a Poisson distribution, ${ }^{[19]}$ with patient demand changing from hour to hour and from day to day. Patients wait for service when the patient demand is greater than the supply. The available resources wait for patients when patient demand is less, resulting in under-utilisation.

Variability is the change in the state of a system or the variation process from time to time. Variability is about the spreading out or the closeness of patients' volume and arrival time throughout the day. A key measure of variability is the ratio of the standard deviation to the mean. The service time needed by a patient from a doctor depends on the patient's medical condition, which further leads to workflow pathways, such as the prescription of drugs, diagnostic tests and surgery. The cycle time differs from patient to patient because the OPC has different types of patients and different pathways. ${ }^{[7]}$ These factors introduce uncertainty in the workflow management. Uncertainty is a state of limited knowledge, where it is impossible to exactly describe the present state and future outcomes of the patient's condition.

OPC management involves scheduling and/or sequencing patients, managing people, equipment, technology, information and all other resources that are needed to deliver the services. The OPC management also affects variability and uncertainty in the workflow. Patients already in the OPC undergo one or the other process and use the required resources. Since there is an interdependency among processes, patients often visit and revisit multiple departments, with high in-process waiting at each department. ${ }^{[20,21]}$ Variability and uncertainty in patients' demand and service time impact care delivery and cause congestion or bottlenecks in OPCs. ${ }^{[20]}$ High variability causes unregulated waiting time at departments and cycle time. ${ }^{[22,23]}$ Variability and uncertainty in the OPCs cannot be eliminated; therefore, this thesis aims to describe ways to effectively manage them.

\section{OPERATING SYSTEM}

Hopp and Spearman in their book "factory physics," define push and pull production systems in the following way: A push system schedules the release of work based on demand, while a pull system authorises the release of work based on system status. ${ }^{[23]}$ In fact, Hopp and Spearman are critical about using "push" and "pull" to describe implemented production/operating systems. The use of "push" and "pull" in the literature often involves a value 
judgement, when authors describe pull systems to contrast them favourably to other systems, which are "not pull" and thus "push." Hopp and Spearman argue that the use of "pull" is broad and diffused, where some authors define "pull" as broadly as "serving the goods that the markets need" and other authors define "pull" as narrowly as "JIT delivery with Kanban." According to Hopp and Spearman, "push" and "pull" should only be used on a conceptual level for gaining an insight into what makes operating systems effective by contrasting extreme push systems with extreme pull systems. ${ }^{[23]}$ The examples mentioned the start of this introduction suggest the following contrasts between an extreme push system and an extreme pull system.

\section{Extreme Push}

Patients are scheduled through appointments depending on the doctor's planned availability. Patients may arrive at the hospital, and no doctor may be available to see them. Care is provided on a predefined fixed schedule, based on the projected patient demand. Upstream departments often transfer patients to downstream departments (according to schedule) without the latter being ready, resulting in unregulated work-in-process (WIP). Since there is no limit on the WIP, corrective actions are taken retroactively for managing the overload of the system, e.g. stopping admissions, unplanned overtime, etc.

\section{Extreme Pull}

Care is provided when the patient asks for it. The information about the availability of resources comes from within the system. Patients are served as much as possible on the first in-first out basis unless this makes the system unstable. Patient demand in OPCS is either known or unknown, and no upstream department should transfer patients to the downstream department until the latter asks for it. This establishes certain WIP limits and also regulates WIP, resulting in lower average WIP level. Little's law states that "The long-term average number of customers in a stable system $L$ is equal to the long-term average effective arrival rate, $\lambda$, multiplied by the average time a customer spends in the system, $W$; or expressed algebraically: $L=\lambda W$." In factory physics, Little's law represents the relation among the three quantities: cycle time, waiting time and throughput. The ratio of WIP to cycle time yields throughput at every WIP level, where the cycle time is directly proportional to WIP. Cycle time $=$ WIP/Throughput. Therefore, lower WIP will result in shorter cycle time. ${ }^{[23]}$

The examples mentioned above show that in "push," there is long latency and a lack of regulation (although the use of planning may suggest otherwise), whereas in "pull" we see short latency and regulation of workflow and resources. For OPC systems with high variability and uncertainty, questions could be raised about the best planning method and when we should plan? Earlier, we wrote that the distinction between push and pull is at the conceptual level 
and we need more specific terminology to describe, analyse and design real-time operating systems. As the target condition is in real-time, we explore how well operations management (planning, scheduling and coordination) in an OPC system could be designed with an optimal mix of push-pull to optimise workflow in real-time.

\section{VARIABLES TO DESIGN AN OPC SYSTEM}

An operating system consists of elements. We define the elements we will use in this thesis to design an operating system as well as to test the hypotheses (Box 1.1). Therefore, we use an example to show how the defined elements fit into the design of the operating systems and the conditions on which it should operate.

With the list of definitions, we are better able to describe and analyse OPCs. We will show this by the example of a rather small OPC. Figure 1.1 shows the operation of functionally organised OPCs that have disparate departments. The departments often take decisions with limited knowledge to optimise the capacity that does not necessarily improve the overall system performance. Adding to this, the average demand-based planning and scheduling does not match real-time demand, due to variability and uncertainty associated with demand and workflow pathways. This type of department-centric (local) optimisation does not work well. Although the shorter reaction time of the departments sometimes improves their efficiency, the larger latency reduces the efficiency. In contrast, patient-flow-centric optimisation with real-time control on operations like sequencing and scheduling by sharing information and shorter latency would benefit the OPC as a whole. Hence, waiting time, cycle time and resource utilisation are improved, along with additional enhancement of profit and patient satisfaction. ${ }^{[24]}$

The basic structure of an OPC is a queuing network with departments, such as D1, D2 and D3, pathways and control C, as shown in Figure 1.1. OPCs are usually modelled by $G / G / n$

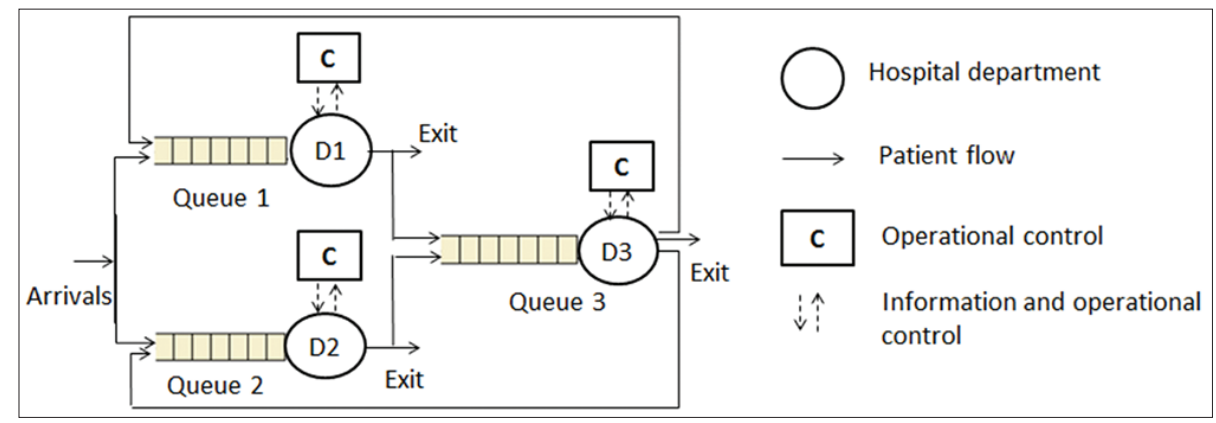

Figure 1.1: Functional and operational basic structure of an outpatient clinic system 
Box 1.1: List of definitions

Aggregate level planning: It is an intermediate-term (3 months-1 year) planning to balance capacity and demand in such a way that costs are minimised.

Arrival time: The time at which a patient enters a hospital.

Assembly line system: A line of workers and equipment along which a product being assembled passes consecutively from one operation to another operation, until it is completed.

Closed loop: A control system in which an operation, process or mechanism is regulated by feedback.

Continuous flow: A streamlined hat involves ongoing delivery of services.

Coordination: The synchronisation and integration of activities and responsibilities to ensure that resources in a system function efficiently.

Cross-skills: To get trained in different skills (more than one skill).

Cycle time: The time spent by the patient in the OPC from entry to exit, constituting of service time, waiting time and travel time.

Decision variable: A variable takes a range of values and helps decision makers to control the parameter of interest. Disparate subsystem: This refers to dissimilar elements/subsystems that are entirely different or dissimilar in their structure, operations and management and yet, part of a larger system.

Downstream process/department: It refers to the process/department that occurs later (towards the end) in a workflow of service delivery or production sequence.

Dynamic environment: An interactive environment that keeps changing and involves conflicting/competing forces.

Efficiency: The comparison of what is actually produced or performed with what can be achieved with the same consumption of resources. It can be defined as the optimum utilisation of inputs at lower costs.

Feedback: Information collected by an entity (individual or a group or a system) about its prior working, to facilitate the modification of the current and future working for achieving the desired results.

Global: A group of subsystems that operate as a whole system.

Latency: The total time (global) to take action in a system, when a change is triggered either by external or internal factors.

Line balance: It is a process of assigning tasks to departments to remove bottlenecks and excess capacity.

Local: Relating or restricted to particular subsystems or units.

Open loop: It does not use feedback to determine if its output has achieved the desired goal of input. It does not observe the output of the processes controlled by it.

Operating system: It is the way a system functions and controls different entities like patients, resources and processes. It is a working method that focuses principally on the cause and effect relationships (or stimulus/response, behaviour, etc.) of specific interest at a particular point in time.

Operational control: It is the control over the execution of normal business processes at the operational level.

Operator load: It is the total work an employee/operator performs depending on his working speed and skills.

Pathways: Routes followed by patients that pass through various departments of outpatient clinics, depending on patients' diagnostic complexities.

Patient: A person receiving or registered to receive medical treatment.

Planning: A process of organising the activities and developing a strategy of future action to achieve the desired set of goals.

Process: Sequence of interdependent and linked procedures that consume one or more resources (employee time, energy, machines, money) to convert inputs (data, material, parts, etc.) into outputs. These outputs then serve as inputs for the next stage, until a known goal or result is reached.

Queuing network: A network of queues is a system in which a number of queues are connected by patient routing. When a patient is serviced at one department, he/she can join the queue at another department or leave the network.

Reaction time: The time for taking actions in a subsystem when change is triggered either by external or internal factors. 


\section{Box 1.1: (Continued)}

Real-time: The actual time during which a process or event occurs.

Resource: Staff that support functioning of the hospital.

Response time: It is the time taken by the scheduler to obtain an optimal path.

Scheduling: The process of converting plan into action based on given information with available resources and time constraints. A schedule can also be defined as a detailed plan of the tasks with respect to time.

Sequencing: Arranging or organising the activities one after the other or in a particular order, usually on the basis of task interdependence.

Service time: It is the time taken for providing care to a patient.

Subsystem: A subsystem is a set of elements, which is a system by itself and a component of a larger system.

System: A system is a set of interacting or interdependent components forming an integrated whole. Every system is characterised by its spatial and temporal boundaries, surrounded and influenced by its structure, behaviour and environment.

Threshold workload: The fixed level of workload that is sustainable or acceptable.

Throughput time: It is the reciprocal of throughput. It is the time at which a patient moves out of the OPC. Throughput: It is the rate at which an OPC system provides care or services. It is the number of patients serviced during a period. It measures the entire system and not a locality.

Uncertainty: A state of limited knowledge, where it is impossible to exactly describe the present state and future outcomes of tasks.

Upstream process/department: The process/department is upstream if it is followed by consecutive processes/ departments in a workflow of supply chain or service delivery.

Utilisation: The proportion of the available time that a resource or a system is operating.

Variability: It is the change in the state of a system or the process of variation, from time to time.

Waiting time: It is the time elapsed between the arrival of a patient and the start of a service or care.

Workflow: Series of activities necessary to complete a task/procedure, consisting of an orchestrated and the repeatable pattern of activities, enabled by the systematic organisation of resources into processes that transform materials, provide services, or process information.

WIP: WIP is partially completed processes or services that are initialised or started, but are not finished. Workload: It is the amount of work an individual, a department or a system has to do.

WIP: Work-in-process

models (general distribution where arrival time and service time processes are both arbitrary with " $n$ " number of servers, arrival $\lambda$ by Poisson distribution and service $\mu$ by an exponential distribution). All queues are first-come first-served, with queue lengths limited by the waiting area. The arrival rates and service rates are $\lambda_{1}, \lambda_{2}, \lambda_{3}$ and $\mu_{1}, \mu_{2}, \mu_{3}$ respectively.

The patients in the OPC follow any of the pathways, D1, D2, D1-D3, D2-D3, D1-D3-D1, D1-D3-D2, D2-D3-D2, D2-D3-D1, depending on the type of care and patients' medical condition. Therefore, selection of the pathway is uncertain. New patient arrivals, along with the re-entry of patients from various departments, increase patient demand and result in variability. The schedules of resources or patients are based on forecasts of average demand. The resources are scheduled according to the forecasts of average demand and availability constraints. ${ }^{[25]}$ The OPCs follow a rule of maintaining a ratio between a fixed number of appointments and walk-in patients for patient scheduling. If the patient demand exceeds 
the planned number (due to walk-ins), the staff extend their working hours, resulting in staff overtime.

Planning and control are local to the departments; hence every department controls its own patient flow with the aim to optimise its capacity and improve its efficiency. Due to the functional structure in an OPC, the departmental decisions are independently taken by managers for achieving department-specific goals, with limited knowledge. ${ }^{[4,5,26]}$ The manager in D1, using the average demand anticipation, negotiates for an extra resource during planning and scheduling, and by obtaining it, D1 treats the patients faster. The patients are released from upstream department D1 to downstream department D3 without knowing the downstream's status. This definitely improves the departmental efficiency and the waiting time of patients in D1. However, D3 receives patients faster from D1 and is not ready to take many patients, as D3 resources are limited. Therefore, D3 has an increase in arrival rate but no change is caused in service rate, resulting in patients waiting longer in D3. D1 improved its patient flow, but its efficiency does not reduce the patient's cycle time. Information about patient demand or available resources is not shared among the departments. This lack of coordination between the departments creates bottlenecks in the OPC, disrupting the patient flow and causing an increase in latency. ${ }^{[6,8]}$ This shows that functional organisational structures often facilitate efficient departments, but not overall OPC efficiency. Due to this, hospitals accept inefficiencies in their organisations, and the department managers' goals (local) do not match with the goals (global) of the hospital/OPC. ${ }^{[6]}$

The optimisation of D1 increased the unregulated WIP in D3. According to the schedule, D3 can manage a certain level of threshold workload ( $t$ ). When the workload in D3 exceeds the threshold $(\mathrm{t})$, the operational control " $\mathrm{C}$ " in D3 identifies the difference between workload in queue and threshold workload, and signals departments D1 and D2 to block the entrance to these two departments. Therefore, according to the queue limit (layout dependent) and supply, the departments can set some rules (WIP cap), based on which decisions can be made. Example: if(Queue 3>t) $\rightarrow(D 1: \downarrow$ and D2: $\downarrow)$. The OPCs often collect feedback after the event (end of the day). Cause and effect are experienced during the same time, whereas action is taken later through planning and scheduling. The latency can be either a day or week or a month. Because of longer latency, the workflow cannot be optimised in the same timescale.

At present, OPCs utilise feedback for future planning and control and this does not influence the current workflow problems. This latency hinders the performance of the OPC system. Therefore, the OPC system requires constant feedback from all subsystems, so that rescheduling is based on when and where the change is triggered and might be accomplished in real-time. The reaction time depends on the flexibility of the subsystems in the OPC, whereas latency depends on the flexibility and coordination in the OPC system. ${ }^{[27]}$ The OPC is an open-loop system that is affected by its surroundings, as there is a delay in utilising 
the feedback and taking action to improve the workflow. It is this latency that categorises the OPC as open loop system rather than closed loop system.

The example shows that local planning and scheduling, based on projected average demand, is inefficient like department D1 causing prolonged waiting time and cycle time. Ideally, care should be provided to the patient when asked for it, i.e., by patient-centric and flow-centric optimisation. The system status might be obtained through continuous feedback (closed system characteristic) with the real-time operating system. The information collected could be used for decision-making at various levels of management based on task granularity and time horizon. The OPC requires rescheduling operations or activities with all of its departments, such that the variability and the uncertainty (open system characteristics) are optimally buffered, and OPC system is generally optimised. Being responsive to a changing demand can be achieved by being flexible enough to accommodate changes in real-time, without latency. Various decisions have different time windows to react to the variations and uncertainties. Operational decisions have a shorter time window (hours-day) to react to the variations. The planning and scheduling are carried out in longer time horizons (weeks-months) and do not incorporate the variabilities and uncertainties in real-time.

The cycle time mainly constitutes of service time, travel time and waiting time. Service time necessarily depends on the medical condition of the patient and the competency of the staff. Deviation in service times is reduced by process standardisation in the OPC, such that each procedure takes the same service time for all patients. Travel time depends on the physical layout of the OPC and cycle time can be minimised by reducing the number of transfers and the distance of departments or locations. Waiting time is the result of the management of patients and resources, and the coordination among departments in the OPC. According to Little's law, the cycle time can be either controlled or minimised by reducing the waiting time, while holding the throughput constant. ${ }^{[12]}$ In the example above, the cycle time is minimised if the total waiting time at all departments in the pathway is reduced. In order to achieve this, departments in the OPC should plan, schedule and coordinate as a system, in real-time. This thesis demonstrates the various methods for optimising patient flow globally (overall) and in real-time to improve the cycle time, waiting time and utilisation of resources in OPCs.

\section{STUDY AIM AND RESEARCH QUESTIONS}

The operational problems in OPCs are caused by the local optimisation (departmental organisation) and the execution of planning and control (average demand-based and not in real-time). Based on this, we state the central hypothesis of the thesis and the research sub-questions. 


\section{Central Hypothesis}

A hospital system with disparate subsystems cannot minimise cycle/waiting time by separately optimising subsystems, or by scheduling that does not adjust in real-time.

And conversely,

A hospital system with disparate subsystems can reduce cycle/waiting time by overall (globally, not locally with respect to department) optimisation and by using real-time scheduling.

In order to test the central hypothesis of the present study, the overall research question is:

How can overall (global) optimisation along with real-time scheduling improve the wait/cycle time and hospital performance?

This research question leads to the following sub-questions, which are explored in this study.

1. Does overall (global) optimisation along with predictive (nearly real-time) resource planning improve waiting time and cycle time?

2. Does overall (global) optimisation along with real-time patient scheduling improve waiting time and cycle time?

3. Does overall (global) optimisation along with real-time coordination of resources and patients improve waiting time and cycle time?

4. What combination of each optimisation model best improves (reduces) the waiting time and cycle time?

\section{OUTLINE OF THE THESIS}

The thesis is organised as follows:

Chapter 2 presents literature survey to identify the gap between what is known and what is not known about planning, scheduling and control in OPCs in real-time. It highlights the various variables used in design operating system. Chapter 3 presents the research methodology, selection of variables, research strategies used, research design and data collection and analysis. In Chapter 4, we present predictive resource planning model that determines the resources required to adapt to patient demand by considering variability and uncertainty, in short-term. We develop a mathematical model based on Takt time management to generate resource plans. We present the simulation results along with the implementation results where the model has been implemented. The effect of reaction time on waiting times and cycle times is also explored. In Chapter 5, we present an integral patient scheduling model 
for minimising the patients' time spent in the OPC. For that, we develop a hybrid ant agent algorithm to minimise patient waiting time. We show the effect of this patient scheduling on waiting times and cycle times through simulation and implementation results. In Chapter 6, we present real-time scheduling of resources and patients and the coordination required to achieve real-time scheduling. The OPC is modelled as a multi-agent system where resources and patients are agents that negotiate and coordinate to achieve their respective goals. We present the simulation and the implementation results (waiting time, cycle time and utilisation). Chapter 7 presents the synthesis and discussion of the main findings of the thesis along with the best mix of optimisation models required for an ideal operating system, followed by study limitations and future research.

\section{REFERENCES}

1. Bergeson SC, Dean JD. A systems approach to patient-centered care. JAMA 2006;296(23):2848-51.

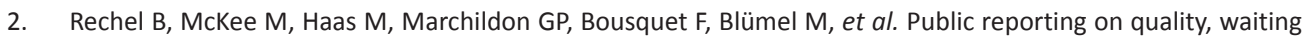
times and patient experience in 11 high-income countries. Health Policy 2016;120(4):377-83.

3. van Merode GG, Groothuis S. Hospitals as complexes of queuing systems. In: Health Sciences Simulation. Orlando, Florida, USA: Society for Modelling and Simulation International (SCS); 2003.

4. Goldschmidt HM, de Vries JC, van Merode GG, Derksc JJ. A workflow management tool for laboratory medicine. Lab Autom Inf Manag 1998;33(3):183-97.

5. van Merode F, Molema H, Goldschmidt H. GUM and six sigma approaches positioned as deterministic tools in quality target engineering. Accredit Qual Assur 2004;10(1-2):32-6.

6. Ludwig M, Van Merode F, Groot W. Principal agent relationships and the efficiency of hospitals. Eur J Health Econ 2010;11(3):291-304.

7. Monreal J Jr, Valerdi R, Latt LD. A systems approach to healthcare efficiency improvement. Procedia Comput Sci 2014;28:610-8.

8. van Merode F. A prelude of the 2004 antwerp quality conference: Targets and target values-integrating quality management and costing. Accredit Qual Assur 2004;9(3):168-71.

9. Bailey NT. A study of queues and appointment systems in hospital out-patient departments, with special reference to waiting times. J R Stat Soc Ser B Methodol 1952;14(2):185-99.

10. Duska LR, Mueller J, Lothamer H, Pelkofski EB, Novicoff WM. Lean methodology improves efficiency in outpatient academic Gynecologic Oncology clinics. Gynecol Oncol 2015;138(3):707-11.

11. Crass M, Munro M. Successful implementation of process review in ophthalmology services. Health Manpow Manage 1997;23(4-5):133-6.

12. Hopp WJ, Spearman ML. Factory Physics: Foundations of Manufacturing Management. New York, NY: Irwin, The McGraw-Hill; 1996. p. 668.

13. Womack JP, Jones DT, Roos D. The Machine That Changed the World. New York: Simon \& Schuster; 1990. p. 352.

14. Liker JK. The Toyota Way: 14 Management Principles from the World's Greatest Manufacturer. New York: McGraw-Hill; 2004. p. 330.

15. Spear S, Bowen HK. Decoding the DNA of the toyota production system. Harv Bus Rev 1999;77(5):96-106.

16. Hopp WJ, Spearman ML. To pull or not to pull: What is the question? Manuf Serv Oper Manag 2004;6(2):133-48.

17. Story P. Dynamic Capacity Management for Healthcare: Advanced Methods and Tools for Optimization. New York: Productivity Press; 2010. p. 226.

18. Palvannan RK, Teow KL. Queueing for healthcare. J Med Syst 2012;36(2):541-7.

19. Hassan MH, Zaghloul AA, Mokhtar SA. The probability distribution of attendance to hospital emergency units 


\section{Chapter 1}

for school students in Alexandria. J Egypt Public Health Assoc 2005;80(1-2):127-51.

20. Noon CE, Hankins CT, Côté MJ. Understanding the impact of variation in the delivery of healthcare services. J Healthc Manag 2003;48(2):82-97.

21. Armistead C, Clark G. The "coping" capacity management strategy in service in services and the influence on quality. Int J Serv Ind Manag 1994;5(2):5-22.

22. Young T, Brailsford S, Connell C, Davies R, Harper P, Klein JH. Using industrial processes to improve patient care. BMJ 2004;328:162-4.

23. Hopp WJ, Spearman ML. Factory Physics: Foundations of Manufacturing Management. New York, NY: Irwin, McGraw-Hill; 2001. p. 698.

24. Fogarty C, Cronin P. Waiting for healthcare: A concept analysis. J Adv Nurs 2008;61(4):463-71.

25. Allder S, Silvester K, Walley P. Managing capacity and demand across the patient journey. Clin Med (Lond) 2010;10(1):13-5.

26. Mintzberg H. Structure in 5's: A synthesis of the research on organization design. Manag Sci 1980;26(3):322-41.

27. Bernardes ES, Hanna MD. A theoretical review of flexibility, agility and responsiveness in the operations management literature toward a conceptual definition of customer responsiveness. Int J Oper Prod Manag 2009;29(1-2):30-53. 




\section{INTRODUCTION}

We present an overview of the related work on resource planning and scheduling, patient scheduling and coordination in outpatient clinics (OPCS). Learning/exploring more about operations management (planning, scheduling and control) of patients and resources (staff) will facilitate the research in finding further ways to optimise the workflow.

\section{Planning, Scheduling and Control in Hospitals}

We now analyse how hospitals plan, schedule and control their operations, the input, output and control variables used in such studies, and what can be used from the literature for designing an operating system that optimises workflow in real-time.

\section{Resource planning}

Planning, scheduling and control of resources, patients, and activities are important tasks that impact waiting time in hospitals. ${ }^{[1]}$ Various researchers have proposed frameworks of hierarchical planning and control in health care. Vissers et al. ${ }^{[2]}$ proposed a planning framework for hospitals consisting of a number of levels (resource, patient and department). The framework assumes a common situation in which a hospital is organised through relatively independent units and resources, and patients are scheduled in isolation. The framework is limited to planning and scheduling that is performed in advance. The number of resources required for a patient group is determined at the aggregate level. Aggregate planning balances the capacity and demand to minimise costs. The term "aggregate" is used because planning at this level includes all resources "in the aggregate." There are business management software like manufacturing resource planning and enterprise resource planning (ERP) that are used in industries to collect, store and manage data from planning, inventory, shipping, etc. Van Merode et al. ${ }^{[3]}$ analysed the use of ERP to hospital systems. These planning systems do not reflect the reality due to variability and uncertainty in demand. The authors suggest that the available planning tools are inadequate for stochastic demand, thereby necessitating the usage of an advanced planning system (APS). APS simultaneously plans and schedules operations based on the available resources and actual demand. It provides visual management, increased throughput, and improved services. ${ }^{[4,5]}$

Demand forecasting is a way to deal with variability and uncertainty. Hospitals plan and schedule resources much ahead of time (weekly or monthly) depending on their expected average demand based on the clinic's experience or through an appointment system. ${ }^{[6-8]}$ Forecasting patient demand is done in short-term like a day, but not shorter than that. Even with daily prediction, the variability remains high. Staff scheduling is often concerned with cost optimisation, utilisation and efficiency, and not with optimisation of waiting time. Mansdorf ${ }^{[6]}$ 
describes resource (staff) scheduling by using a mathematical model for a multispecialty clinic that included various types of care, staff mix, staff working hours and overtime. The staff scheduling is based on the average deterministic demand and remains fixed for a month. It does not incorporate the short-term demand variability. Another study by Hulshof et $a{ }{ }^{[9]}$ adapted resources to deterministic demand (not stochastic) in the longer time horizon (week). Waiting time and cost were the parameters of interest. Although the resources were scheduled on the basis of the patient demand, they did not capture the variability and uncertainty in the short-term. Demand forecasting models used in the hospitals predict demand over a long time horizon and such models do not capture variability in the short-term, hence the existing capacity planning does not work. ${ }^{[10]}$ Resource planning and scheduling are strategic and tactical decisions that fail to incorporate operational level information.

Staff scheduling using analytical models is not sufficient as they do not capture real life scenarios. ${ }^{[6,9]}$ Therefore, hospitals, as well as researchers, use simulation models to study the effect of all significant variables on the patient flow in a department or the entire hospital. The individual parameters are adjusted through a series of simulations, providing the management with valuable material to support its decisions in a complex clinical production with constant demands for higher efficiency in several areas. Integrating simulation modelling and optimisation has a great potential to improve outcomes. ${ }^{[8,11-13]}$

Baril et $a l .{ }^{[8]}$ conducted a study in an orthopaedic OPC, where the patients were scheduled through appointments 3 months in advance, making the patient demand deterministic. The resources such as consulting rooms, nurses and orthopaedists were scheduled on a weekly basis. A model was built on the basis of a real case study, utilising empirical data to represent real service time, patient arrival times, doctor lateness and absence. It focused on scheduled patients as it had only a few daily walk-in patients. Service times and arrival rate were the independent variables, and the performance measures were resource utilisation, number of patients treated and patient lead time..$^{[8]}$ The hospitals schedule their resources on the basis of the average deterministic demand. ${ }^{[14]}$ Harper $^{[12]}$ highlighted the need to capture variability in patient demand and complexity of the hospital system. A generic framework for operational modelling of hospital capacity was proposed, and a planning and management tool called patient and resource operational management planning tool (PROMPT) was developed using this framework. The tool incorporated statistical analysis of data followed by a simulation model of the hospital. It classified the patients into different groups and calculated the number of beds in daily use, the number of refusals and utilisation. The tool provided insight on the short-term variability in demand, but it depended on appointments.

OPCs often consider delays as capacity problems. Slack planning of capacity strategies creates additional time-slots by overestimating a number of patients at the tactical level, and these capacities are used to schedule surgeries at the operational level. When demand is 
lesser than expected, the time-slots remain idle. It improves the waiting time but is counterproductive to hospital efficiency. ${ }^{[1]}$ Rouppe van der Voort et al. ${ }^{[15]}$ demonstrated that the delays (waiting times) are caused by the system design resulting in a poor matching of supply and demand. Various ways were used to match the supplies with deterministic demand (through appointments) for a single department to improve delays in access to care.

The study conducted by Vermeulen et al. ${ }^{[16]}$ in a computed tomography scan OPC shows that matching the supply to patient demand reduces the waiting time. The patient demand was deterministic, as patients were scheduled 2 weeks ahead through a resource calendar, with the capacity allocated per patient group. No walk-in patients, no shows and machine downtime were considered. The working hours extended on the basis of the patient demand and the staff was informed a week before. A large planning window (days-weeks) has less variability and can make optimal use of the surplus capacity, whereas a small planning window, with fewer patients and larger variability, needs more surplus resources. Resource rescheduling was not dynamic. Stochastic patient arrivals for 20 weeks were simulated to analyse the effect of scheduling. The study coordinated resource scheduling and patient scheduling. Scheduling was performed for a single procedure or a department, not for multiple departments.

Van Merode ${ }^{[17]}$ describes hospitals as functional structures that include several care stages performed by various health care professionals. The efficiency and effectiveness of the hospital is a result of the planning and control decisions made for care services at each stage. Departments are often concerned with maximising the efficiency of their processes. Thus, they put their professionals and resources at the centre and organise everything around them, not around the patient. The departmental goals differ from that of the hospital. ${ }^{[18]}$ Due to the interdependence of departments, fluctuations in either patient arrivals or resource availability or both in one department may affect the entire patient pathway. This may result in varying waiting times for the patients at different stages of the care pathway. From a hospital perspective, this may result in varying resources' utilisation. The local management of patients and resources in departments leads to sub-optimisation, which is a threat as the capacity decisions are taken in isolation and not in an overall hospital perspective. ${ }^{[18,19]}$ Hospitals have tried to adapt production control principles to optimise their performance, but these are applied to the independent business units and not to the system as a whole. ${ }^{[20]}$

The literature shows that hospitals often match the demand with supplies. The resource planning is based on the deterministic average patient demand in longer time horizons (weeksmonths). However, there is always a chance of deviation in reality that gives rise to variability and uncertainty, and OPCs fail to incorporate it during planning. Additionally, most resource planning and scheduling models are department-centric (local), not hospital-wide. The local 
optimisation of departments results in bottlenecks in the patient flow. Hospitals schedule patients (pre-scheduled or walk-in) for care along with the resources. Next, we study the impact of patient scheduling on the performance of hospitals.

\section{Patient scheduling}

In general, patients are scheduled based on the availability of resources (doctors and equipment) with the goal of matching the patient demand with supply, most often to maximise resource utilisation and profit. Often, patient scheduling deals with appointment scheduling. ${ }^{[1-24]}$ In the literature, we notice that the appointment systems have evolved from manual methods to automated methods. ${ }^{[25-29]}$ The first published work on patient scheduling was by Bailey who attempted to balance the patient and doctor waiting times through queuing models. ${ }^{[23]}$ Unfortunately, the queuing models were insufficient to incorporate complexities within hospitals, whereas simulation modelling captured workflow complexities. Therefore, various scheduling policies were analysed by simulation modelling to improve resource utilisation and waiting times. ${ }^{[22]}$ Appointment scheduling considers no shows, cancellation, double booking and time-slot allocation for walk-in patients. ${ }^{[21,24,26,30-32]}$ OPCs reserve certain time-slots for walk-in patients based on the demand forecast. ${ }^{[3-37]}$ Forecasting walk-in patient demand improves scheduling. ${ }^{[3,34]}$ However, the studies that included walk-in patients in patient scheduling had considered a few number of walk-in patients. ${ }^{[8,26,35]}$

The only study that considered a high percentage (72\%) of walk-in patients in a clinic applied four types of mixed registration strategies. ${ }^{[36]}$ One of these minimised the waiting time and throughput time (cycle time) for scheduled patients, while another minimised the same parameters for walk-in patients. This study provides effective management of time-slots for registration of both types of patients, but the walk-in patients were not pre-planned in real-time. Different strategies were tried, and one of them worked for that particular situation. There was no mathematical model that could be generalised for other hospitals. The study was only for the registration department.

In literature, the research on patient scheduling has mainly dealt with optimising appointment scheduling. The patients are independently scheduled in different departments because of department-centric control in OPCs. Because of local control, variability and uncertainty, some departments are overcrowded and some remain idle. Patients wait in queues of the overcrowded departments and are unaware of their pathways and the waiting status of other departments. With increased complexities in diagnoses and treatment, patients might take complex pathways and visit multiple departments. Patients are often scheduled without considering a complete patient pathway, because of which they repetitively wait at various departments. 
It is also observed that disparate sub-systems in OPCs manage patient scheduling and resource scheduling in isolation. ${ }^{[2]}$ The goals of the patients, resources, departments and management differ from each other. Therefore, a question of interest is whether OPC performance can be improved if resources, patients and their scheduling are managed in an integrated fashion. For this, the OPC systems require real-time coordination among departments for planning, scheduling and controlling of activities. We now examine the type and the time of coordination required to improve OPC's performance.

\section{Coordination in hospitals}

Coordination often relates to appointment scheduling and treatment follow-up activities of patients. ${ }^{[38]}$ Brailsford et al. ${ }^{[39]}$ states that effective interactions between departments improve workflow, utilisation and provide quality care. The importance of coordination and communication in process operations was demonstrated by the use of an electronic whiteboard in emergency and inpatient departments. ${ }^{[40,41]}$ The framework of Vissers et al. is limited to planning and scheduling at a strategic and tactical level. The planning and scheduling of resources and patients are performed at different levels (hence are isolated) and lacks a coordination mechanism at the operational level. ${ }^{[2]}$

The analytical models are inadequate for modelling interactions in hospitals, and therefore, a methodology called "multi-agent systems" (MASs) is used. This methodology is effective and efficient in handling interactions in distributed and dynamic environments. ${ }^{[42]}$ Formally, the MAS is composed of multiple interacting agents within an environment. All the staff, resources, managers and patients represent the different types of agents with predefined goals, which interact and cooperate to collectively improve their state. ${ }^{[43]}$ This system has been used in the modelling of emergency departments, operation theatres and inpatient hospitals. ${ }^{[4,45]}$ Paulussen et al. ${ }^{[46,47]}$ describe patients and resources as agents and show that scheduling and coordinating the patients increases efficiency. Patient agents compete for time-slots of scarce resources. All the above studies performed simulation modelling to look at an optimal solution using the coordinating agents. However, the output was given to the departments to let them adopt. These models do not have feedback and optimisation in real-time.

Decker ${ }^{[48]}$ developed a coordination mechanism that modelled the hospital as MAS, with different rule-based agents bidding for resources or time-slots. Generalised partial global planning (GPGP) is a scheduling coordination approach that provides a planner to create task structures aiming for achieving agent goals and a scheduler that attempts to maximise utility. A GPGP approach along with MAS modelling reduced the patient's stay (cycle time) and increased throughput in a spatially distributed hospital. Deshpande et al. ${ }^{\left[{ }^{[9]}\right.}$ extended the GPGP approach by providing a coordination mechanism for resource sharing across hospitals 
with multiple objectives like quality, cost and duration for optimisation, which reduced the complexity. Štiglic and Kokol ${ }^{[50]}$ modelled hospitals as MAS to monitor and forecast patient demand for a week, based on which the resource scheduling was adapted for that week. A scheduling agent evaluated the available time-slots and waiting times before fixing appointments for patients to reduce the average waiting times.

Vermeulen et al. ${ }^{[51]}$ proposed multi-agent Pareto appointment exchanging for patient scheduling. The patients' schedule is improved by virtual agents, who negotiate and exchange appointments to reduce the waiting time. Zöller et $a{ } .{ }^{[52]}$ describe patient scheduling that allows them to bid for the earliest treatment based on the resources that are auctioned. Agents representing the patients compete for treatment appointments in the fictitious marketplace. The resource agents auction off time-slots, and if a resource is free, its next time-slot is assigned to the patient agent with the highest bid. Each patient agent determines the benefit of treatment as the price it is willing to pay. The utility of a resource is defined according to how much it improves a patient's health. This approach reduced waiting times and improved resource occupancy in the hospital, but failed to incorporate scheduling of walk-in patients.

As seen in the literature, scheduling of patients and resources has been performed in isolation. A study conducted by White et al. ${ }^{[53]}$ shows that integration of patient scheduling, capacity scheduling and patient flow improves the patients' experience and the clinic's operational performance. ${ }^{[53]}$ This study describes capacity allocation, various appointment scheduling policies and different patient flow configurations, but does not optimise its performance in real-time. Despite the potential importance of coordination and integration of these issues, we find surprisingly few studies on this.

\section{CONCLUSION}

The aim of this literature review was to analyse the methods and techniques used for planning, scheduling and control of patients, resources and activities. More specifically, we wanted to identify significant variables to design an operating system that improves OPC performance by global and real-time scheduling.

Operations' modelling in hospitals has been researched extensively in the recent past. The resource planning is based on the deterministic and average demand over a long time horizon. The variability and uncertainty in actual demand widen the gap between planning and reality. The current planning methods do not incorporate variability and uncertainty during planning. Patient scheduling often deals with appointment scheduling. There is no literature that schedules the patients in real-time. Furthermore, patients and resources are scheduled separately without real-time coordination. 
In order to sum up, the OPC systems consist of disparate sub-systems that are locally managed and controlled because of which, there are no globally optimising operations management systems. In addition, it is not known how OPCs plan, schedule and control patients and resources in real-time. Therefore, we conclude that there is a need to analyse how to design an OPC system that optimises its patient flow and resources in real-time and globally through resource planning, patient scheduling and coordination mechanism and by incorporating variability and uncertainty, thereby improving the performance measures, namely, waiting time, cycle time and utilisation. We present the selection of design variables used to design an operating system in this study in Chapter 3.

\section{REFERENCES}

1. Dellaert N, Cayiroglu E, Jeunet J. Assessing and controlling the impact of hospital capacity planning on the waiting time. Int J Prod Res 2016;54(8):2203-14.

2. Vissers JM, Bertrand JW, de Vries G. A framework for production control in health care organizations. Prod Plann Control Manag Oper 2001;12(6):591-604.

3. van Merode GG, Groothuis S, Hasman A. Enterprise resource planning for hospitals. Int J Med Inform 2004;73(6):493-501.

4. Lee $\mathrm{YH}$, Jeong CS, Moon C. Advanced planning and scheduling with outsourcing in manufacturing supply chain. Comput Ind Eng 2002;43(1-2):351-74.

5. Chen K, Ji P. A mixed integer programming model for advanced planning and scheduling (APS). Eur J Oper Res 2007;181(1):515-22.

6. Mansdorf BD. Allocation of resources for ambulatory care -a staffing model for outpatient clinics. Public Health Rep 1975;90:393-401.

7. Yurko LC, Coffee TL, Fusilero J, Yowler CJ, Brandt CP, Fratianne RB. Management of an inpatient-outpatient clinic: An eight-year review. J Burn Care Res 2001;22(3):250-4.

8. Baril C, Gascon V, Cartier S. Design and analysis of an outpatient orthopaedic clinic performance with discrete event simulation and design of experiments. Comput Ind Eng 2014;78:285-98.

9. Hulshof PJ, Mes MK, Boucherie RJ, Hans EW. Tactical Planning in Healthcare using Approximate Dynamic Programming; 2013.

10. Kepner EB, Spencer R. Planning staff and space capacity requirements during wartime. US Army Med Dep J 2016;(2-16):124-7.

11. Swisher JR, Jacobson SH. Evaluating the design of a family practice healthcare clinic using discrete-event simulation. Health Care Manag Sci 2002;5(2):75-88.

12. Harper PR. A framework for operational modelling of hospital resources. Health Care Manag Sci 2002;5(3):165-73.

13. Kittipittayakorn C, Ying KC. Using the integration of discrete event and agent-based simulation to enhance outpatient service quality in an orthopedic department. J Healthcare Eng 2016;2016:8.

14. Nguyen TB, Sivakumar Al, Graves SC. A network flow approach for tactical resource planning in outpatient clinics. Health Care Manag Sci 2015;18(2):124-36.

15. Rouppe van der Voort MM, van Merode FG, Berden BH. Making sense of delays in outpatient specialty care: A system perspective. Health Policy 2010;97(1):44-52.

16. Vermeulen IB, Bohte SM, Elkhuizen SG, Lameris H, Bakker PJ, Poutre HL. Adaptive resource allocation for efficient patient scheduling. Artif Intell Med 2009;46(1):67-80.

17. van Merode F. A prelude of the 2004 antwerp quality conference: Targets and target values-integrating quality management and costing. Accredit Qual Assur 2004;9(3):168-71. 
18. Ludwig M, Van Merode F, Groot W. Principal agent relationships and the efficiency of hospitals. Eur J Health Econ 2010;11(3):291-304.

19. Hans EW, van Houdenhoven M, Hulshof PJ. A Framework for health care planning and control. In: Handbook of Healthcare Systems Scheduling. Vol. 168. New York: Springer; 2012.

20. Duska LR, Mueller J, Lothamer H, Pelkofski EB, Novicoff WM. Lean methodology improves efficiency in outpatient academic gynecologic oncology clinics. Gynecol Oncol 2015;138(3):707-11.

21. Mageshwari G, Kanaga EG. Literature review on patient scheduling techniques. Int J Comput Sci Eng IJCSE 2012;4(3):397-401.

22. Harper P, Gamlin HM. Reduced outpatient waiting times with improved appointment scheduling: A simulation modelling approach. OR Spectr 2003;25(2):207-22.

23. Bailey NT. A study of queues and appointment systems in hospital out-patient departments, with special reference to waiting times. J R Stat Soc Ser B Methodol 1952;14(2):185-99.

24. Cayirli T, Veral E. Outpatient scheduling in health care: A review of literature. Prod Oper Manag 2003;12(4):519-49.

25. Byron HM. The ophthalmologist's office: Planning and practice. Effective patient scheduling. Int Ophthalmol Clin 1975;15(2):85-93.

26. Berg BP, Denton BT, Erdogan SA, Rohleder T, Huschka T. Optimal booking and scheduling in outpatient procedure centers. Comput Oper Res 2014;50:24-37.

27. Friedman JP. Internet patient scheduling in real-life practice. J Med Pract Manage 2004;20(1):13-5.

28. Legrain A, Fortin ME, Lahrichi N, Rousseau LM. Online stochastic optimization of radiotherapy patient scheduling. Health Care Manag Sci 2015;18(2):110-23.

29. Azadeh A, Baghersad M, Farahani MH, Zarrin M. Semi-online patient scheduling in pathology laboratories. Artif Intell Med 2015;64(3):217-26.

30. Giachetti RE. A simulation study of interventions to reduce appointment lead-time and patient no-show rate. In: Proceedings of the 2008 Winter Simulation Conference. Miami, FL: IEEE Press; 2008. p. 1463-8.

31. Kaandorp GC, Koole G. Optimal outpatient appointment scheduling. Health Care Manag Sci 2007;10(3):217-29.

32. Zeng B, Turkcan A, Lin J, Lawley M. Clinic scheduling models with overbooking for patients with heterogeneous no-show probabilities. Ann Oper Res 2010;178(1):121-44.

33. Holleman DR, Bowling RL, Gathy C. Predicting daily visits to a walk-in clinic and emergency department using calendar and weather data. J Gen Intern Med 1996;11(4):237-9.

34. Cayirli T, Gunes ED. Outpatient appointment scheduling in presence of seasonal walk-ins. J Oper Res Soc 2014;65(4):512-31.

35. Peng Y, Qu X, Shi J. A hybrid simulation and genetic algorithm approach to determine the optimal scheduling templates for open access clinics admitting walk-in patients. Comput Ind Eng 2014;72(1):282-96.

36. Su S, Shih CL. Managing a mixed-registration-type appointment system in outpatient clinics. Int J Med Inf 2003;70(1):31-40.

37. Kortbeek N, Zonderland ME, Boucherie RJ, Litvak N, Hans EW. Designing cyclic appointment schedules for outpatient clinics with scheduled and unscheduled patient arrivals. Perform Eval 201480:5-26.

38. Conforti D, Guerriero F, Guido R. Optimization models for radiotherapy patient scheduling. 4OR 2008;6(3):263-78.

39. Brailsford SC, Sykes J, Harper PR. Incorporating human behavior in healthcare simulation models. In: Proceedings of the $38^{\text {th }}$ Conference on Winter Simulation. Monterey, California; 2006.

40. Wong HJ, Caesar M, Bandali S, Agnew J, Abrams H. Electronic inpatient whiteboards: Improving multidisciplinary communication and coordination of care. Int J Med Inf 2009;78(4):239-47.

41. France DJ, Levin S, Hemphill R, Chen K, Rickard D, Makowski R, et al. Emergency physicians' behaviors and workload in the presence of an electronic whiteboard. Int J Med Inf 2005;74(10):827-37.

42. Bogg P, Beydoun G, Low G. When to Use a Multi-Agent Systems? In Intelligent Agents and Multi-Agent Systems. Berlin, Heidelberg: Springer; 2008. p. 98-108.

43. Williamson M, Decker KS, Sycara K. Executing decision-theoretic plans in multi-agent environments. In: AAAI Fall Symposium on Plan Execution. Cambridge, Massachusetts: AAAI Press; 1996.

44. Schmidt R, Geisler S, Spreckelsen C. Decision support for hospital bed management using adaptable individual 


\section{Chapter 2}

length of stay estimations and shared resources. BMC Med Inform Decis Mak 2013;13:3.

45. Paulussen TO, Zöller A, Heinzl A, Braubach L, Pokahr A, Lamersdorf W. Patient scheduling under uncertainty. In: Proceedings of the 2004 ACM Symposium on Applied Computing. Nicosia, Cyprus: ACM; 2004.

46. Paulussen TO, Zöller A, Heinzl A, Pokahr A, Braubach L, Lamersdorf W. Dynamic patient scheduling in hospitals. Coordination and Agent Technology in Value Networks. Berlin: GITO; 2004.

47. Paulussen TO, Jennings NR, Decker KS, Heinzl A. Distributed patient scheduling in hospitals. In: Proceedings of the Eighteenth International Joint Conference on Artificial Intelligence (IJCAI-03). Morgan Kaufmann; 2003.

48. Decker K. Coordinating intelligent agents. In: Foundations and Applications of Multi-agent Systems. Berlin, Heidelberg: Springer; 2002. p. 1-18.

49. Deshpande U, Gupta A, Basu A. A distributed hospital resource scheduling system using a multi-agent framework. lete Tech Rev 2001;18(4):263-75.

50. Štiglic G, Kokol P. Intelligent patient and nurse scheduling in ambulatory health care centers. In: Engineering in Medicine and Biology $27^{\text {th }}$ Annual Conference. Shanghai, China; 2005.

51. Vermeulen I, Bohte S, Somefun K, Poutré HL. Multi-agent pareto appointment exchanging in hospital patient scheduling. Serv Oriented Comput Appl 2007;1(3):185-96.

52. Zöller A, Braubach L, Pokahr A, Rothlauf F, Paulussen TO, Lamersdorf W, et al. Evaluation of a multi-agent system for hospital patient scheduling. Int Trans Syst Sci Appl 2006;1(4):375-80.

53. White DL, Frohle CM, Klassen KJ. The effect of integrated scheduling and capacity policies on clinical efficiency. Prod Oper Manag 2011;20(3):442-55. 




\section{INTRODUCTION}

In this chapter, we present the variables used, the research strategies and design, the settings where the study was conducted, the design/plan, population and the samples. Ethical considerations, data collection, analyses and the research flow are described.

\section{VARIABLES}

Initially, we did exploratory research to identify the variables through focus group discussions with the staff and doctors, who were the service providers in the hospital under study. In addition, we described the literature (Chapter 2), with attention to the variables that different researchers have used and considered these for our own choice. Further, we have verified these variables with the administrators and managers of the hospital. We have selected the following dependent variables (also called output variables): Waiting times, cycle times and utilisation.

There are two types of independent variables, also referred to as input variables: Controllable and uncontrollable. The controllable independent variables in this study are resource scheduling, patient scheduling and coordination mechanism. The uncontrollable independent variables are independent of each other and cannot be manipulated in real-world experiments; however, they can be changed in simulation models. The uncontrollable independent variables in this study are patient volume, arrival times, patient types, service times and skill levels/cross-skills. We cannot optimise values of these in the hospital itself, but we can optimise the values of the controllable variables to give best results over a range of plausible uncontrollable inputs. We select reaction time as an uncontrolled independent variable that gets affected by internal and external environment (also referred as an exogenous variable). In addition, there are independent variables like patient age and gender, which do not affect or change the workflow or dependent variables and are extraneous variables in this study.

\section{RESEARCH STRATEGY}

In this research, the workflow of the hospital and its characteristics need to be first studied in detail. In order to capture the finer and minute details about the variables and the constraints that affect the output, the workflow needs to be analysed.

With the help of this understanding, a simulation model can be developed. Simulations can generally be conducted faster than in real-time and allow experimentation with different values of the uncontrollable variables. Various experiments in different scenarios can be 
conducted by using the simulation model. In order to observe the impact of interventions in the real environment, the optimisation models need to be implemented in the hospital settings. The implementation of the research or optimisation models in real-time is the final stage of this research. Therefore, we characterise the research strategy into three facets: A case study, model building and implementation.

\section{Case Study}

For the preliminary exploratory stage, we used a case study to investigate a contemporary phenomenon within its real-life context. A case study is useful in the early stages of research when a fresh perspective is needed. Its strength is its ability to deal with a full range of evidence: Documentation, artefacts, interviews and observations. We examined an outpatient clinic (OPC) system over a period to understand its working conditions/environment. This provided realistic information related to the problem under consideration. Workflow optimisation in the OPC involves many complexities in patient pathways, patients and resources management and various administrative issues. In order to record the fine details of the variations, our case study provided a strong foundation. It captured the holistic characteristics of real-life events during the investigation and their interdependencies.

\section{Model Building}

A simulation model of workflow management was developed from the details obtained during the case study. The model was built using exploratory data analysis to understand the relationships between the input and output/dependent variables. It included the formulation/specification of the processes through which the values of variables change over time and represent the original/actual situation of the OPC. It utilised inputs, outputs, processes, constraints, feedbacks and environment that were explored during data collection. The study replicated the existing workflow of the OPC model and validated it for its correctness. Within this model, each of the optimisation/intervention models was applied, and the output/ dependent variables were measured. The outcomes were analysed, and recommendations were deduced.

\section{Implementation}

Implementation of research in real-life deals with various issues related to patients, staff, management and information technologies (IT). The optimisation/intervention models were integrated into the hospital management system software. The data from the hospital database was utilised to test the models' workability in a real-time system. The model's compatibility with other functions of the software was tested. The models were implemented for 2 months in the OPC of Aravind Eye Hospital (AEH), after which the data relevant to the study 
was collected to validate the optimisation models. Further, the data before implementation and after implementation were compared and recommendations were made. In addition, the opinion of the staff, doctors and administrators about their experience on waiting times were collected.

\section{RESEARCH DESIGN}

This study used a quantitative descriptive experimental design to identify, analyse and describe the factors contributing to prolonged waiting times and cycle times in OPCs.

\section{Quantitative Research}

This study attempted to quantify the factors identified as contributing to waiting times and cycle times in the OPCs. The variables that we quantify are patient volume, arrival times, service times, number of resources, patient age, waiting times, cycle times and utilisation. Gender and clinical pathways are categorical; hence, they are not quantified.

\section{Descriptive Research}

The study explored the factors influencing the waiting times and cycle times in OPCs. It attempted to identify new meanings and insights. This research attempted to investigate the full nature of the phenomenon (waiting times), the manner in which it manifests and propagates and the related factors that could influence it. This aims to identify accurate characteristics of the real-life situations in the OPC. A case study in the natural setting of an OPC provided detailed information on the processes.

\section{Experimental Research}

Experimental research is a systematic and scientific approach to research that manipulates one or more variables and controls and measures any change in other variables. This study manipulated the independent controllable variables (planning and scheduling rules of resources and patients and coordination between them) by experimenting with a range of values for independent uncontrollable variables like different patient volume, arrival pattern/distribution and service times, to observe the effect on dependent variables: Waiting times and cycle times (Figure 3.1). The independent uncontrollable, controllable and extraneous variables are the inputs to the OPC model, and dependent variables are the measured outputs.

Stratified random sampling was used to select the experimental design. The monthly patient demand for a year was analysed. The observation and analysis of the data collected 


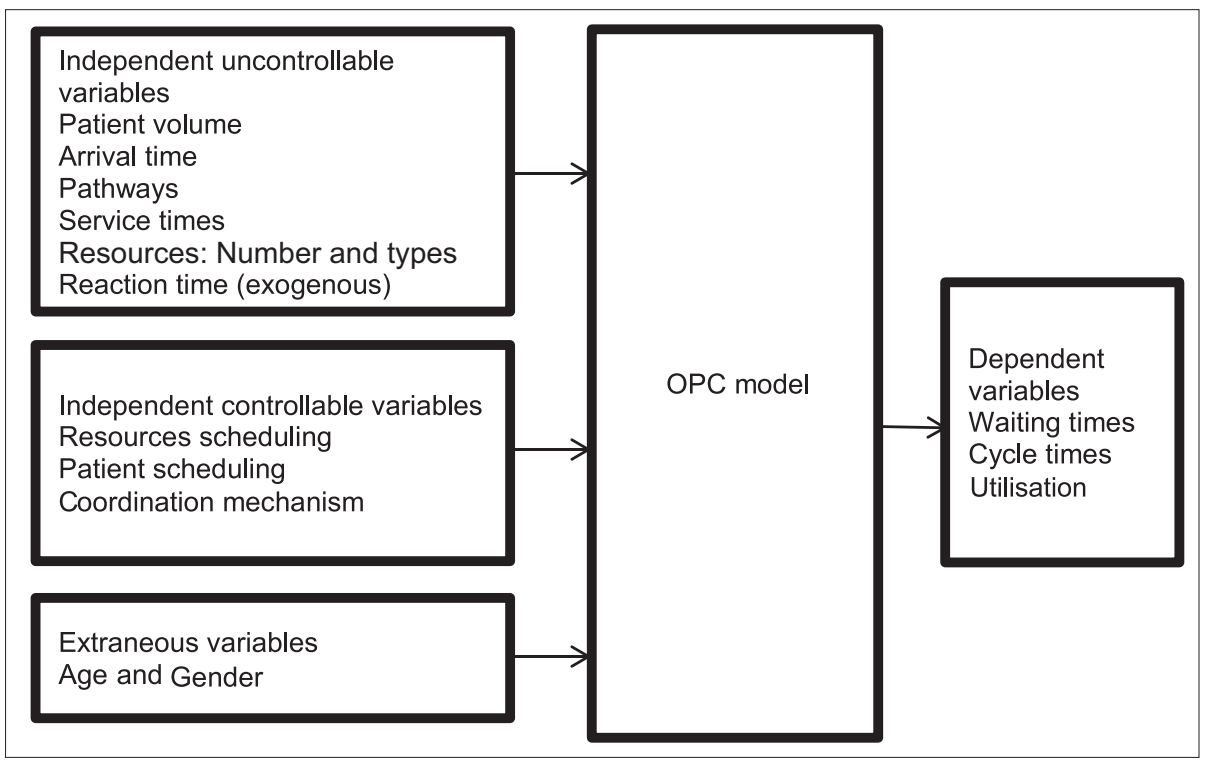

Figure 3.1: Relations between variables

helped in analysing the variability in the patient demand over a period of 1 year. No 2 days received the same patient demand. It was observed that around $30.2 \%$ was between 1000 and 1600 patients/day, 53.4\% was between 1600 and 2000 patients/day and $16.4 \%$ was >2000 patients/day. Based on this analysis, the patient demand/load was categorised into three groups: Low, medium and high. Different arrival times were generated for the same mean patient arrivals. The same randomiser input was used for simulation with the two different scenarios: Current rules and proposed real-time rules. This assured that the results obtained were not due to randomness. The patient category is one factor with three levels. Reaction time plays an important role in waiting time, and we selected three levels for this. The levels were $r(t 1) \leq 10 \mathrm{~min}, 11 \leq r(t 2) \leq 20 \mathrm{~min}$ and $21 \leq r(t 3) \leq 30 \mathrm{~min}$. The reaction times for different departments were randomly assigned to the mentioned level. Therefore, two factors with three levels were selected (Table 3.1). The experiments were conducted with the existing and the proposed models. Full factorial experiments were carried out to estimate the effect of selected factors on performance parameters. This was the basic framework for the simulation model. It was further adapted to suit specific research questions and is described in detail in the subsequent chapters.

Figure 3.2 shows a schematic representation of simulation experiments. Experimental designs were replicated to estimate the variability associated with the phenomenon. The mean and standard deviation of the dependent variables were collected after the simulation run was over. The simulation was stopped with the completion of replications of the experimental designs. The study examines the influence of different variables and interventions on waiting 


\section{Chapter 3}

Table 3.1: Experimental factors

\begin{tabular}{ll}
\hline Factors/independent variables & Setting \\
\hline Patient class & 1. Low \\
& 2. Medium \\
Reaction time $r(\mathrm{t})$ in min & 3. High \\
& $\leq 10$ \\
Intervention & $11-20$ \\
& $21-30$ \\
\end{tabular}

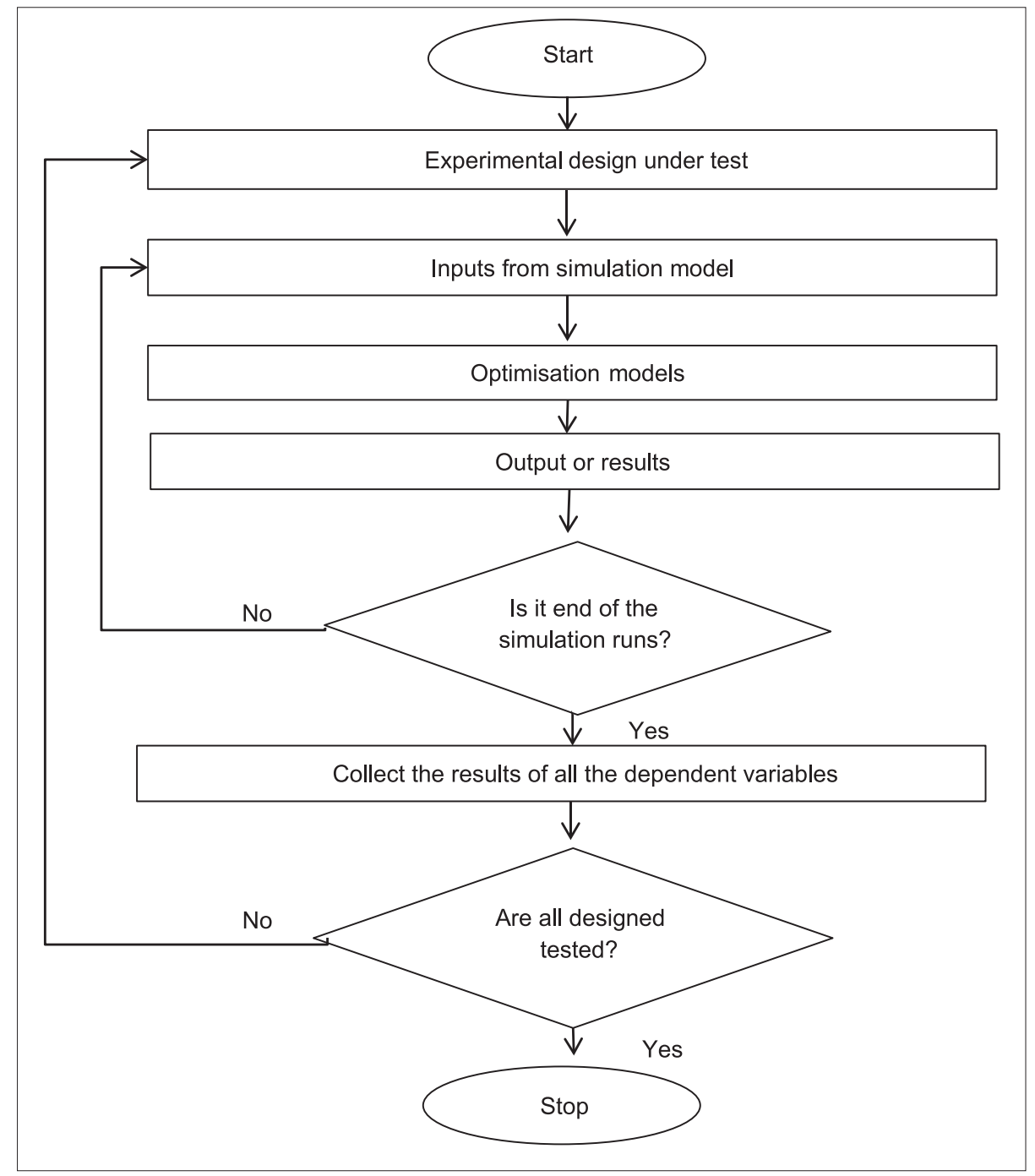

Figure 3.2: Simulation experiments 
times and cycle times by simulation and field experiment. The dependent variables, waiting times and cycle times were measured before and after optimisation or intervention to accept or reject the hypothesis. The level of significance is determined by $P$ value in statistical tests. A $P=0.05$ was selected, meaning that for $P<0.05$ the null hypothesis is rejected and the alternative hypothesis is accepted.

\section{RESEARCH HYPOTHESES}

The research questions have been stated earlier in Chapter 1 and now we state the research hypotheses. Each hypothesis is tested in Chapters 4-6, respectively.

1. An OPC system with disparate subsystems can reduce cycle/wait time by global optimisation and real-time resource planning, using robust predictive resource planning

2. An OPC system with disparate subsystems can reduce cycle/wait time by global optimisation and real-time patient scheduling

3. An OPC system with disparate subsystems can reduce cycle/wait time by global optimisation and coordination among resources and patients, in real-time.

\section{RESEARCH SETTING}

In a general hospital, a patient may have any ailment specific to one part of the body. This will be identified at the first level of screening and the patient is sent to the concerned department. Similar to a general hospital, even in an eye hospital, patients are first screened and sent to the concerned specialty clinic.

In India, out of several eye hospitals, AEH, Madurai (Tamil Nadu, India) has a name for quality. The hospital attains its goals by strict attention to cost optimisation and high quality through its operational excellence. ${ }^{[1-4]} \mathrm{AEH}$ is an integrated hospital[ ${ }^{[5]}$ with standardised operations and the services are focussed on patients. AEH focusses on the maximum utilisation of resources. With $<1 \%$ of the country's ophthalmic workforce, AEH accounts for $5 \%$ of ophthalmic surgeries performed nationwide. AEH provides specialised services in large volumes. It is the world's largest hospital that performed 401,529 surgeries and treated 2,396,864 outpatients during 2014-15..$^{[6-8]}$ The hospital uses a manual scheduling system and local optimisation to manage its workflow. Patients are provided care by means of first-come first-served basis at the registration. We studied the real-time global optimisation in this high volume hospital that does not use appointments and provides care to the patient on the same day. A detailed working of AEH OPC is provided in the respective chapters. 


\section{RESEARCH POPULATION AND PARTICIPANTS}

The OPC in AEH is the research population for this study. The hospital has four units in the OPC that are categorised on the basis of the patient age: Unit 1 and Unit 2 (>35 years), Unit 3 (16-35 years) and Unit 4 (<15 years). Units 1 and 2 received more patients than the other two units. These units were identical in their structure with the same number of resources scheduled in each unit. It is also interesting that both the units maintained equal workload and we chose these units for this study.

The research dealt with operational problems; therefore, the participants involved in this study were patients, staff, managers, administrators, paramedical staff, doctors and people working in the IT department.

\section{DATA COLLECTION AND ANALYSIS}

The initial data were collected by observing and interviewing patients and hospital staff, such as doctors, paramedical staff, managers and administrative staff, to understand the workflow process. The empirical data from the Integrated Hospital Management System (IHMS) and the Clinical Management System (CMS) software were used to obtain data relevant to the study's research questions. The relevant data were extracted and reported by using Microsoft Excel software. Statistical data like mean and standard deviation were analysed and obtained from the empirical data. A data-fitting tool, Easy Fit, was used to estimate the probability distribution of service time and patient arrival time. The goodness of fit test was conducted by using the Kolmogorov-Smirnov test. Analysis of variance (ANOVA) with $P=0.05$ was conducted on the dependent variables like waiting time and cycle time to identify statistically significant effects. ANOVA was selected because we were interested in the difference in performance between the existing OPC model and the interventions.

\section{ETHICAL CONSIDERATIONS}

The study did not include any of the patients directly. The research was related to operations management; therefore, the data were collected from the IHMS and CMS from the hospital database. Patient data related to logistics, namely, in-time and out-time, age and type were extracted from the database. Confidentiality was maintained as no personal data, such as patient names and addresses, were extracted. The scheduling affected the patients' experience positively in the OPC. A few patients waited longer than they would have without the intervention, but this was a small percentage of patients. 


\section{RESEARCH FLOW}

After data collection and its analysis, the research steps followed are shown in Figure 3.3. Based on the data collected during case study and empirical data, a simulation model of the OPC was developed. ${ }^{[9-14]}$ The model was validated by using the mean and standard deviation of the dependent variables, namely, waiting time and cycle time. Further, the research developed three optimisation models to test the hypotheses. Each of the optimisation models was applied (one at a time first and then together) to the AEH simulation model and run by manipulating (by using designs) the independent variables. Results of each model were analysed and compared with the empirical data. The optimisation models were implemented in the OPC of AEH for 2 months each and data was collected later. The dependent variables were compared with the existing and optimised models, and statistical tests were conducted for significance analysis.

In Chapter 4, we present predictive resource planning to test the first hypothesis and answer the first research question. In Chapter 5, we present integral patient scheduling to test the second hypothesis and answer the second research question. In Chapter 6, we present

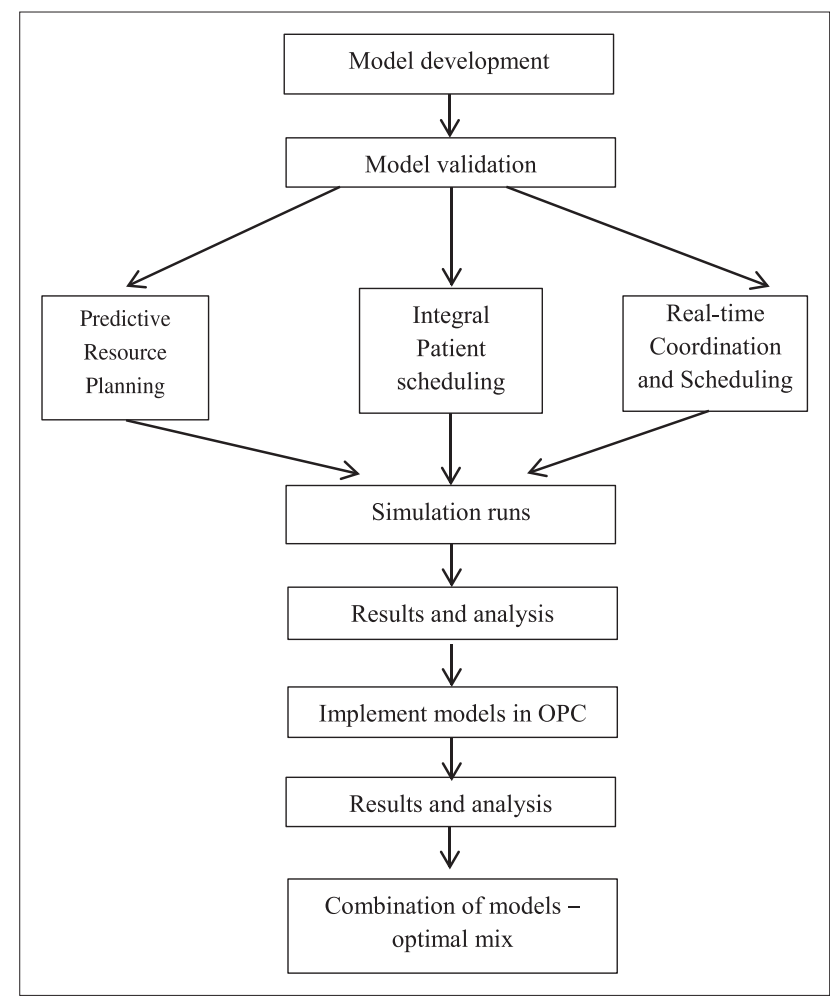

Figure 3.3: Research flow chart 
real-time scheduling and coordination of resources to test the third hypothesis and answer the third research question. In Chapter 7, we mix different combinations of the models to obtain an optimal mix.

\section{REFERENCES}

1. Chaudhary B, Modi AG, Reddy K. Right to sight: A management case study on Aravind Eye Hospitals. Zenith Int J Multidiscip Res 2012;2(1):447-57.

2. Priyadarshini RG. Eye care for All - A Case on Aravind Eye Care Systems. Coimbatore: Society for HRM (SHRM), School of Business; 2011.

3. Brilliant L, Brilliant G. Aravind: Partner and social science innovator (Innovations case discussion: Aravind Eye Care System). Innov Technol Governance Global 2007;2(4):50-2.

4. Molema JJ. Hospital System Design: Creating Supply Flexibility to Match Demand Variability. Maastricht, The Netherlands: Department of Health Organization, Policy and Economics, CAPHRI, FHML, Maastricht University; 2009. p. 153.

5. Van Merode F. A prelude of the 2004 Antwerp Quality Conference: Targets and target values-integrating quality management and costing. Accred Qual Assur 2004:9(3):168-71.

6. Ramani KV, Mavalankar DV, Govil D. Strategic Issues and Challenges in Health Management. India: SAGE Publications; 2008. p. 227.

7. Prahalad CK. The Fortune at the Bottom of the Pyramid. Revised and Updated $5^{\text {th }}$ Anniversary Edition: Eradicating Poverty Through Profits. New Jersey: Wharton School Publishing; 2009. p. 432.

8. Aravind Eye Care System: Activity Report. Madurai; 2014-15. p. 76.

9. Harper P, Gamlin HM. Reduced outpatient waiting times with improved appointment scheduling: A simulation modelling approach. OR Spect 2003;25(2):207-22.

10. Costa AX, Ridley SA, Shahani AK, Harper PR, De Senna V, Nielsen MS. Mathematical modelling and simulation for planning critical care capacity. Anaesthesia 2003:58(4):320-7.

11. Jahangirian M, Naseer A, Stergioulas L, Young T, Eldabi T, Brailsford S, et al. Simulation in health-care: Lessons from other sectors. Oper Res 2012;12(1):45-55.

12. Van Berkel PT, Blake JT. Quantitative modelling for wait time reduction: A comprehensive simulation applied in general surgery. Germany: VDM Verlag Dr. Muller; 2008. p. 92.

13. Van Lent WA, Van Berkel P, Van Harten WH. A review on the relation between simulation and improvement in hospitals. BMC Med Inf Decis Making 2012;12(1):18.

14. Law AM, Kelton WD. Simulation Modeling and Analysis. New York: McGraw-Hill; 2000. p. 760. 



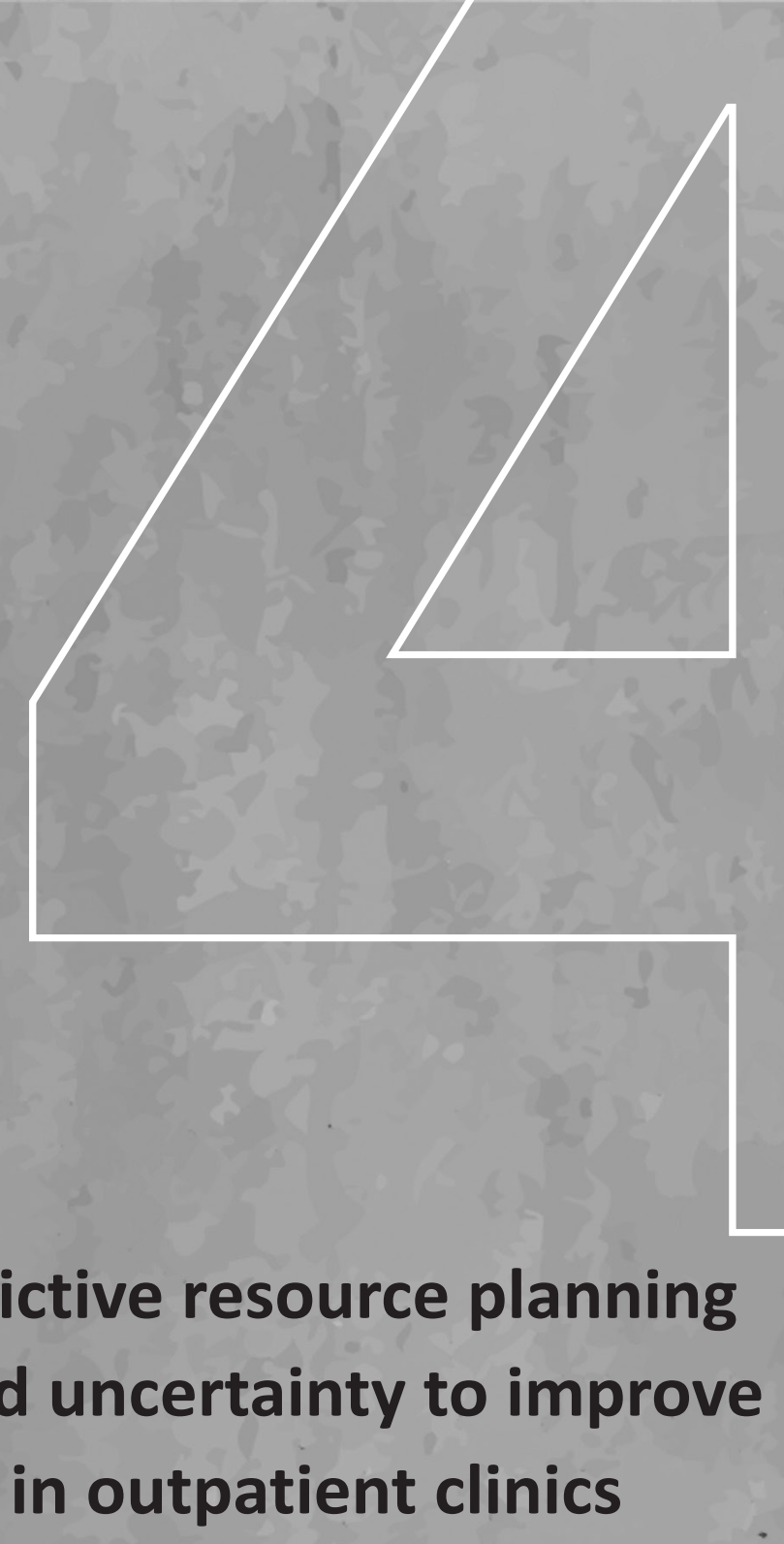

The chapter is published as:

Munavalli JR, Rao SV, Srinivasan A, Manjunath U, van Merode GG. A Robust Predictive Resource Planning Under Demand Uncertainty To Improve Waiting Times In Outpatient Clinics. J Health Manage 2017;19(4):21-30. 



\section{INTRODUCTION}

Resource planning and control have become extremely important in operations management of outpatient clinics (OPCs). Owing to expanding patient demand, greater patient expectations and increasingly complex patient flow, OPC systems are under constant pressure to provide quality care despite limited resources. ${ }^{[1-3]}$ Timely care is a measure of health-care quality that concerns many countries. Patients' waiting time is the major reason for complaints, and patient satisfaction plays a crucial role in quality management. ${ }^{[1]}$

OPCs face operational problems because of the variability and uncertainty in demand and service times, and resource planning is often based on aspects like (1) average patient demand, (2) resource scheduling performed ahead of time and (3) local optimisation. In most of the OPCs, resources are planned and managed through a simple deterministic approach using average demand and service times. ${ }^{[4]}$ Patient arrivals are not uniform and follow Poisson distribution as the patient demand keeps changing from hour to hour. ${ }^{[5]}$ Often, OPCs fail to incorporate or overlook such short-term variability and uncertainty (throughout the day) during planning. ${ }^{[6]}$ As a result, OPCs face inefficiencies, long patient waiting and cycle times and resource under-utilisation, which in turn affects patient satisfaction adversely. ${ }^{[7]}$

The number of resources required in an OPC is determined at an aggregate level planning. ${ }^{[8]}$ The OPCs forecast demand based on experience, historical data or sometimes, advanced analytics. Low demand clinics plan the resources bi-monthly; whereas, high demand clinics plan monthly or weekly. ${ }^{[9,10]}$ Planning depends on the long-term forecasts while the variability in demand occurs in the short term. OPCs, which are open loop systems, are influenced by their environment and experience variability and uncertainties that are caused by late, early or random patient arrivals, varied service times and unpredictable clinical pathways. ${ }^{[7]}$ Resource planning, on the basis of incomplete demand information, does not entirely reflect the reality. The mismatch between planning and reality results in either long waiting time or under-utilisation of resources. Often, OPCs view these frustrating delays as a capacity problem, but the delays are caused likely by poor capacity or resource management. ${ }^{[11]}$ Seasonal variations are predicted and managed by increasing the capacity (for example, part-time doctors)..$^{[12,13]}$

The disparate departments of the OPCs plan and control their operations at the departmental level (local). Every department controls its patient flow and resources. Decisions are often taken without coordination with other departments, because of which patients from the upstream departments are pushed to the downstream departments, which are not ready to service them. Thus, patients wait in some departments and resources remain idle in other departments. Ludwig et al. provide insight into the relationship between departmental and hospital efficiencies. Local optimisation in the departments might improve departmental 
efficiency, but does not necessarily improve OPC-wide efficiency. ${ }^{[14]}$ The departmentally optimised OPCs match patient demand with their services, while patient-centric OPCs match their resources to patient demand. Van Merode et al. suggests the use of short-term planning when the demand is nondeterministic. ${ }^{[15]}$

The commonly used planning and control approaches are inadequate as they are not demand-driven and lack synchronisation. Therefore, there is a need to design an OPC system that synchronises patient flow between the departments and determines the service pace based on actual patient demand with minimum waiting time. We propose a robust predictive resource planning where resources are planned to adapt to demand variability in the short term (hour-hour) and synchronise patient flow between the departments by global planning, rather than simply reacting or responding to whatever comes their way. ${ }^{[16]}$ It means that an OPC system that services patients based on system status or actual demand (pull system) is better than the one based on projected or average demand (push system). ${ }^{[17]}$

Toyota Production System (TPS) is a world leader in industrial production. It utilises a combination of push and pull to reach and maintain a continuous process flow to reduce WorkIn-Process (WIP). ${ }^{[18-20]}$ TPS considers time as a systemic variable associated with the flow of materials and control of operations. ${ }^{[21]}$ TPS applies "Just-In-Time" for a system and not for a single department. That is, a department should not work either slower or faster than the other departments in the line. TPS sets a pace for product flow by applying Takt time management. "Takt time" is derived from the German word Taktzeit for pace or rhythm. It is the desired time between the units of output, to be synchronised with the customer demand. TPS plans, schedules and controls its resources, raw materials, etc., around the required Takt time. TPS also applies line balancing (dividing workload as evenly as possible) to increase the overall productivity. ${ }^{[22-24]}$ Takt time can be used in a production management system termed as "Takt time management." This system fits well in assembly line systems with few product types, known (stable or even) demand, flexible and multi-skilled workforce, single routing and identical work times. As Takt time-based system is demand-driven, it eliminates overtime and overproduction and stabilises the system.

The OPC in Aravind Eye Hospital (AEH) was a good test case for this study as it fits into the assumed working conditions. It is department-centric and determines the resources much ahead of time, based on average demand. AEH has a resemblance to an assembly line system (a line of workers and equipment along which a product being assembled passes consecutively from one operation to another until it is completed). ${ }^{[25-28]}$ The patients in the OPC move through various departments that perform specific and successive tasks. However, some aspects of the OPC, such as local control of operations in the departments and uneven patient demand, differ from assembly line systems. The patient arrivals are random (no appointment system is used) and independent, which makes the patient demand highly variable and 
uncertain. Additionally, it has no control on input and constraints on output, as all patients visiting the OPC are attended to on the same day. ${ }^{[29,30]}$ Therefore, there is a need to adapt Takt to account for this variability. Takt time management is used for global optimisation in manufacturing industries, where industry standard for all assembly lines is maintained and not applied in the short term. Next, we present the resource planning in the OPC and then state the hypothesis of this study.

\section{Resource Planning and Scheduling in OPC of AEH}

AEH is a renowned eye care hospital in Madurai, South India that provides patient-centred care. ${ }^{[25,31,32]} \mathrm{AEH}$ has performed 401,529 surgeries and treated 2, 396, 864 outpatients during 2014-15. ${ }^{[33]}$ The hospital runs with assembly line efficiency, strict quality norms, standardisation, cost control and above all, a high patient volume. The resources (ophthalmologists and paramedical staff) are well trained and dedicated. The OPC predicts its patient demand, which is used for the decisions on staff costing, recruiting and creating awareness among managers. The OPC is open from $7 \mathrm{am}$ to $6 \mathrm{pm}$ and follows a "zero at 10" rule, according to which the patients who arrive before $9 \mathrm{am}$ are to be attended by $10 \mathrm{am}$. This is achieved by transferring the resources with same skills from other clinics to the OPC on the basis of their availability.

The functional and operational structure of the OPC in AEH with respect to resource scheduling is shown in Figure 4.1. Accordingly, in this study, we consider an OPC with two identical units. Departments, such as new registration (NR) and review registration (RR) are common to both units, and each unit has five departments, namely, vision (V), refraction (RF), tension (TN), dilatation (DL) and preliminary and final examination (PE and FE). For clarity, only unit 1 is shown in Figure 4.1. All the queues (1-7) are on the first-come first-served basis. The patient flow arrows show the possible pathways for new and review patients.

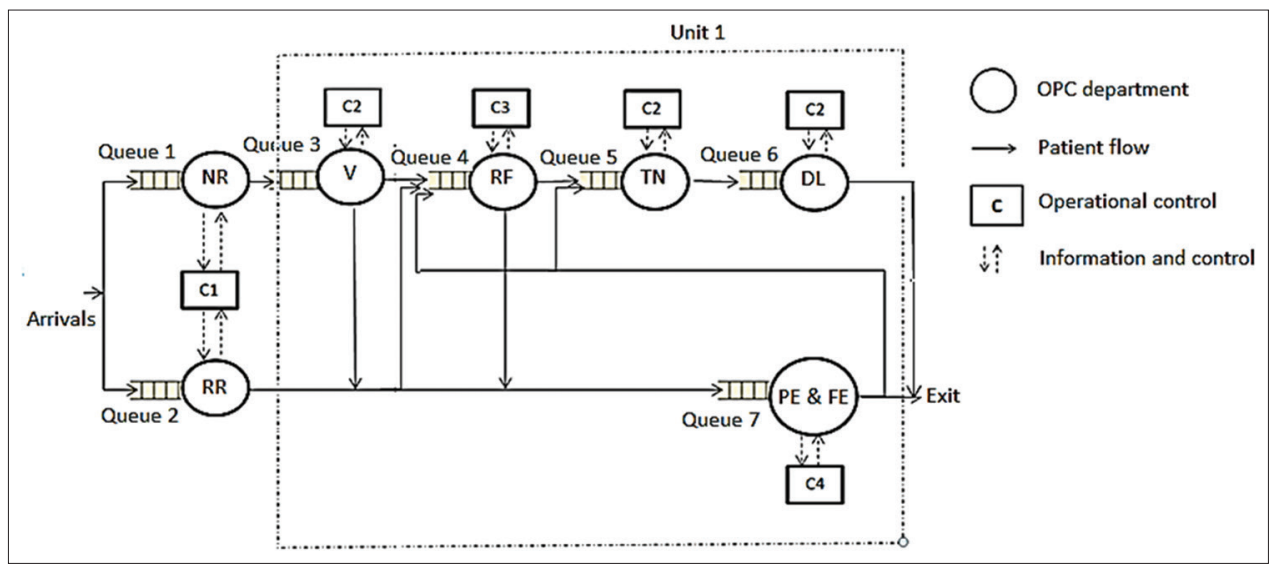

Figure 4.1: Functional structure and operational control in the outpatient clinic system of Aravind Eye Hospital 
The OPC in Figure 4.1 functions according to the rules in Box 4.1. Managers are the controllers (C) who plan, schedule and control the activities of the departments (local). Different managers are responsible for scheduling the resources $(r)$ like ophthalmologists and paramedical staff. The OPC schedules the ophthalmologists once a month, considering their availability after academic (teaching and research) activities and surgery schedules.

Departments of both the units have the same number of resources, which remains fixed throughout the day and month (Rule 3). During peak hours, managers (controllers) apply Rules 4 and 7 to the situation. Based on their experience, they increase resources in their departments or shorten the lunch breaks of the staff to control waiting times, $w(t)$. When upstream departments work faster, the patients flood the downstream departments, which are unready to handle the increased workload. Similarly, when upstream departments work slower, the downstream departments wait for patients. The lack of coordination among the departments results in unregulated waiting time and under-utilisation of resources.

The patient workflow in the OPC starts with registration and finishes with the final examination. At any point in a day, a patient is in waiting, processing or finishing state. The patient moves through various departments by pathways: NR-V-PE-RF-TN-DL-FE for new patients and RR- PE-RF-TN-DL-FE for review patients. The order of the departments RF and PE can be interchanged. Around $5 \%$ of the total number of patients exit after the PE. We define five states (in perspective of operations) for a department. S1 $\rightarrow$ initial state, S2 $\rightarrow$ waiting state, $\mathrm{S} 3 \rightarrow$ regular state, $\mathrm{S} 4 \rightarrow$ reactive state and S5 $\rightarrow$ finish state. Figure 4.2 shows the transition of a department in different states.

The department is in the initial state S1 at the time $t=0$ (start of the day), when patients have not arrived yet. The department moves to the waiting state $\mathrm{S} 2$ at $t=\mathrm{T}$ if either the patient waits for resources or vice-versa. The department is in the regular state S3 when patients arrive at $t=\mathrm{T}$ and are serviced by resources. During peak time, due to high patient

Box 4.1: Rules followed in OPC in AEH

Rule 1: All patients are provided with the service/care on the same day of arrival.

Rule 2: All the queues are FCFS.

Rule 3: Resource planning and scheduling time window is 1 month.

Rule 4: Number of resources is fixed throughout the day and month.

Rule 5: Managers schedule the resources.

Rule 6: Number of resources in unit 1=Number of resources in unit 2.

Rule 7: If (Queue $n>$ threshold workload) $\rightarrow$ (Resources ' $r$ ' is added to $n^{\text {th }}$ department $D_{n}$ such that $r \leq R_{T}$, the total number of resources else shorten the lunch times of the already working resource.

Rule 8: Patients should complete RF and PE before TN.

Rule 9: Control of operations is local (department-centric).

FCFS: First come first serve, OPC: Outpatient clinic, AEH: Aravind Eye Hospital 


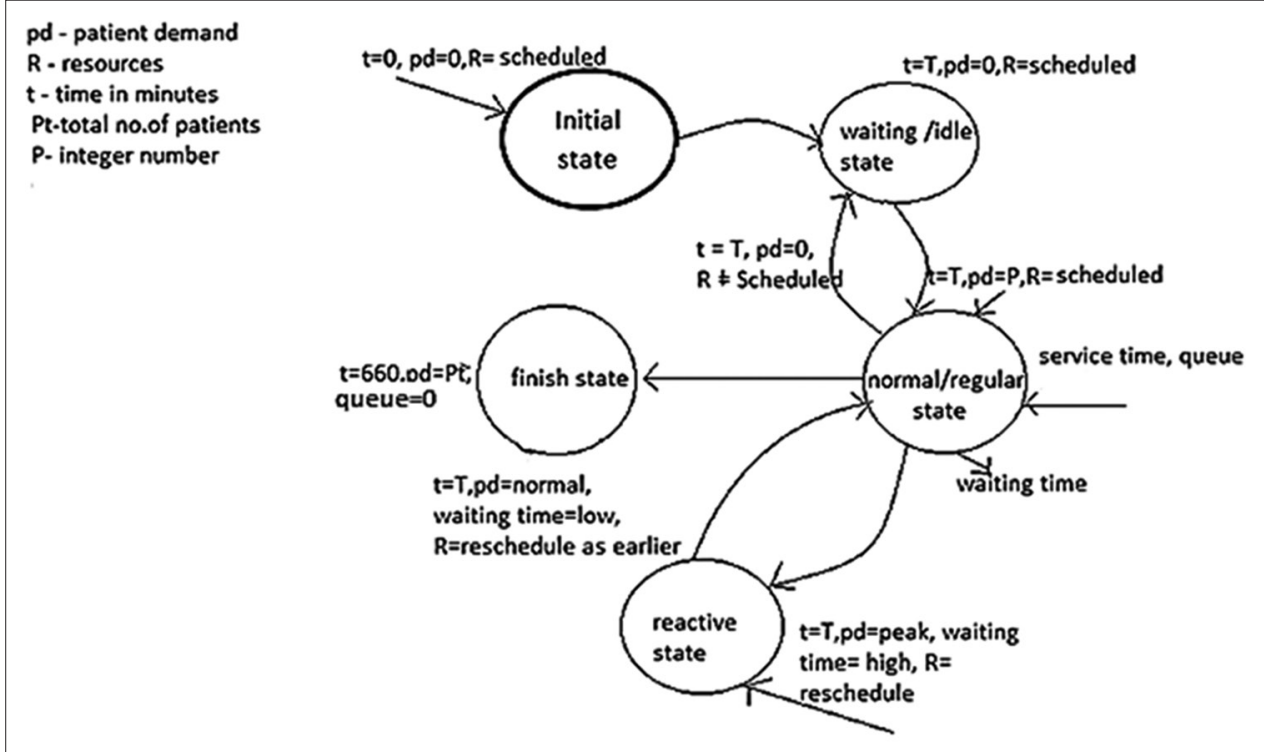

Figure 4.2: State transition diagram for the department

demand, the department has the maximum WIP that the OPC can handle. Now, the department moves to reactive state S4. The manager who controls the operations in the departments responds to the change with limited knowledge (local and available) and follows Rule 7. Once the patient demand becomes regular, the department moves from $\mathrm{S} 4$ to regular state S3, and at the end of the day when all patients are serviced, the department moves to finish state $\mathrm{S} 5$.

The time taken by the manager to change the state of the department from regular to reactive (S3-S4) is the reaction time $r(t)$, which affects the waiting time. The reaction time varies when the resources to be transferred to departments are busy elsewhere. Therefore, the reaction time depends considerably on the resource availability at the time of need and the kind of measures taken. The corrective measures improve waiting time in the departments, but not necessarily the cycle time of patients.

Although the OPC in AEH is efficient, it faces operational problems like long waiting and cycle times and under-utilisation of resources. The OPC, like other hospitals, (Chapter 2, literature review) is an open-loop system with uneven demand, lack of synchronisation between departments and absence of demand-driven resource planning. The resources are scheduled once a month, based on the average projected demand (push) within the individual departments. Therefore, in order to improve its operations, the OPC should determine the service pace based on actual demand (real-time) for all departments. Therefore, 
we develop a robust predictive resource planning, which globally matches service pace of all departments to the varying patient demand. The proposed planning accounts for the variability in patient demand in the short term. Further, the Takt time management is applied to the short-term demand and resources are scheduled accordingly to maintain patient flow. This system is similar to the fast food chains where the raw materials are stored on the basis of projected demand (push), while burgers are prepared on customers' order (pull). In this approach, we integrate global planning with almost real-time (near to actual demand) planning.

We state the hypothesis of this study:

"An OPC system with disparate subsystems can reduce cycle/waiting time by global optimisation, using robust predictive resource planning."

The remainder of this paper is organised as follows: In Section 4.2, we present a robust predictive resource planning model that incorporates short-term demand variability and allocates resources to all departments such that they are synchronised through Takt time management and a balanced patient flow. In Section 4.3, we present materials and methods that include data collection and analysis, model development, experimental design and simulation study. In Section 4.4, we report the results from different scenarios created on real case studies. Then we discuss our findings in Section 4.5 followed by a conclusion in Section 4.6.

\section{PREDICTIVE RESOURCE PLANNING MODEL}

The predictive resource planning model aims to match the resources with short-term demand (hour by hour) for better planning and prepares the OPC to handle the patient flow optimally. This model gives a margin and degree of freedom to keep the OPC working, even when the reality is different from the plan. The predictive resource planning model uses three levels of control (Figure 4.3). In the first level, the daily patient volumes and patient arrival patterns are forecasted by using a forecast generator. The patient volume is predicted on the basis of seasonal variations, such as vacations, short holidays and festivals; while, patient arrival patterns are based on historical data. In the second level, a pace or Takt is set on the basis of the forecast data in the short term. A resource planning model identifies the resource requirements of all departments throughout the day on the basis of patient demand, constraints on resources and precedence. This is a global control measure and the resource plan is shared with all departments. Finally, resources are planned to match the service with arrival rate, i.e., resources are matched to patient demand. In order to implement the predictive resource planning model, we propose a few changes in the rules of the OPC (Box 4.2). 
Box 4.2: Rules proposed by predictive resource planning model to OPC

Rule 3: Resource planning and scheduling time window is every day/one day.

Rule 4: Resources are scheduled every hour.

Rule 5: Resources are scheduled by predictive planning model and managers must only follow the instructions.

Rule 9: Control of operations with respect to resources is global (OPC centric).

OPC: Outpatient clinic

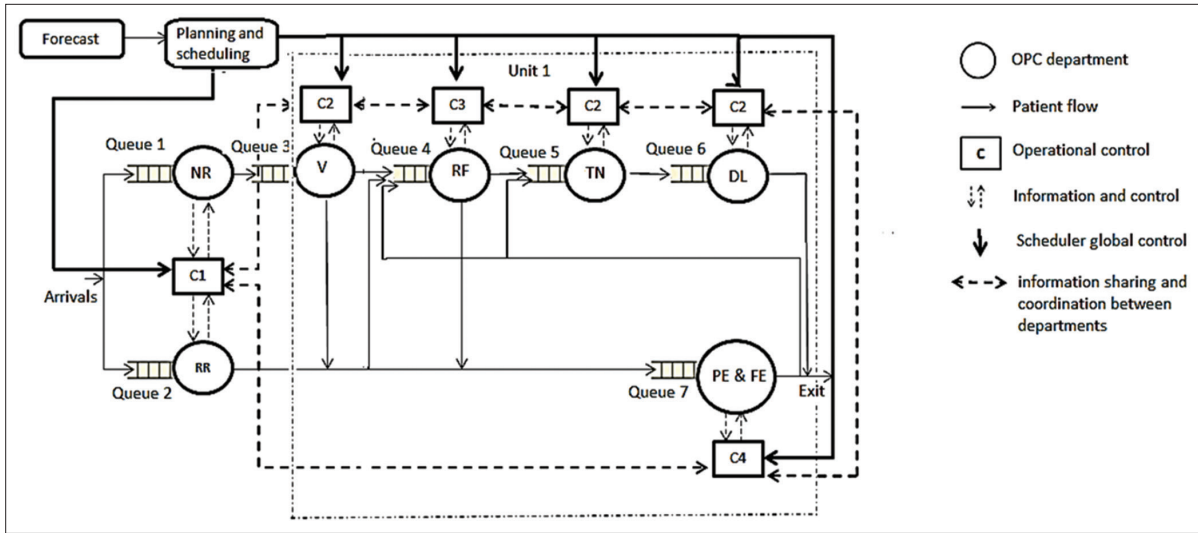

Figure 4.3: Functional structure and operational control of predictive resource planning and scheduling in outpatient clinic system

We modify the rules related to resources and their control but retain other rules 1, 2, 6 and 8 of the OPC system. The planning time window has been changed from a month to a day (Rule 3). Resources are scheduled every hour, and not fixed over a day and month (Rule 4). The resource planning is performed by a planning and scheduling model which accounts for the variability in demand (Rule 5). This eliminates Rule 7 on the manual corrective measures taken by managers. Control of operations remains local, except for resource planning. Now, the managers in the departments do not take the resource planning and scheduling decisions but follow the schedule given by the predictive planning model. The scheduling of resources is not department-centric but OPC-wide.

In the proposed model, patient flow remains the same but the departments have four states instead of five as shown in Figure 4.2. The states are S1-Initial state, S2-waiting state, S3-regular state and S4-finish state. The predictive planning model develops a resource plan on the basis of demand forecast and schedules resources to all departments accordingly. The model centrally determines the global resource schedules, which are applied locally. There is no reactive state in the proposed model, as in the OPC (AEH) model. Instead, there is reaction time to follow the schedule, which is very important. In order to plan better and schedule resources globally, we use Takt time management in the predictive resource planning model. 


\section{Takt Time Based Predictive Resource Planning Model}

Takt time in the OPC context can be explained as the average time at which a patient moves out of the OPC.

Takt time $=\frac{\text { Effective available time in a day }}{\text { Number of patients serviced in a day }}$

In order to understand Takt time management, we consider an example of a system with two departments $A$ and $B$ with mean service times of $10 \pm 2$ and $15 \pm 4$ min respectively. The demand is assumed to be 150 patients/day, and the system is assumed to work for $12 \mathrm{~h}$. There are two breaks of $1 \mathrm{~h}$ each; therefore, the effective work duration will be $10 \mathrm{~h}$ (600 $\mathrm{min})$. The total cycle time will be $25 \pm 6 \mathrm{~min}$ (Here, the cycle time indicates time from the start to the end of service. Some authors use it to indicate the process time on one machine). The Takt time will be $(10 \mathrm{~h} * 60 \mathrm{~min}) / 150=4 \mathrm{~min}$ per patient. The Takt time of $4 \mathrm{~min}$ does not mean that the patients are treated for only 4 min (contradicting the service times in departments $A$ and $B$ ), but it implies that a patient should move out of the system in every 4 min. If the Takt time is $<4 \mathrm{~min}$, then the service in the department is faster than the patient demand, and the resources either wait or are idle. The patient waits if the Takt time is more than 4 min. In order to achieve a Takt of 4 min, departments A and B need resources as calculated in Equation 4.2.

Number of resources $r=\frac{\text { Service time }}{\text { Takt time }}$

We discuss in detail the stochasticity associated with service times later in this section. In this example, we use only mean service time to calculate a number of resources. Therefore, department A will need 10/4 = $2.5 \approx 3$ resources and department B will need 15/4 = 3.75 $\approx 4$ resources to keep the pace of the system. A system would work smoothly with this number of resources if the patient demands were stable or uniform. However, patient arrivals in OPCs are random and uncertain. Patient demand shoots up during peak hours while being less variable during the rest of the time. Thus, setting a single Takt time for a day does not capture the problems of variability and uncertainty in a shorter time horizon, like few hours in a day. Therefore, working hours in a day are divided into " $\mathrm{m}$ " time-slots. Patient arrivals are forecast by using the historical data, arrival times are generated, and the number of patients per time-slot is identified. Further, the Takt time per time-slot is determined by using Equation 4.1. The Takt time remains fixed for a time-slot and varies between time-slots depending on demand; whereas, the cycle time remains the same. By setting pace (Takt) in each time-slot, we match service rate (w.r.t departments) or throughput rate (w.r.t the OPC system) with patient demand throughout the day. Takt time thus sets a real target for improvement. 
Along with demand variability, OPCs also encounter process variability and it is important to consider it in the Takt time design. The effective time taken to perform a task is the service time $s(t)$. Occasionally, the performance of the task is interrupted by a problem which occurs with a probability $\mathrm{p}$. Some amount of time (referred to as surplus time $\mathrm{sp}(\mathrm{t})$ ) is required to solve this problem. ${ }^{[34,35]}$ Therefore, the overall processing time until the task is completed is $\mathrm{p}(\mathrm{t})=\mathrm{s}(\mathrm{t})+\mathrm{p} \mathrm{sp}(\mathrm{t})$. For example, when a certain patient at a department requires more than the average service time, the excess time spent cannot be made up for by attending another patient for a lesser time. Therefore, the surplus time above the average service time should be included in the Takt time design. The squared coefficient of variation of service times (Equation 3 ) includes the effects of surplus time, setup times, irregularities, etc. The mean processing time is $E[p(t)]=E[s(t)]+p E[s p(t)]$. Variance of $p(t)$ is var $s(t)+p$ var $s p(t)+p(1-p)$ $(E[s p(t)])^{2}$. The squared coefficient of variation of processing time $p(t)$,

$$
C_{p(t)}^{2}=\frac{\operatorname{var} s(t)+p v a r s p(t)+p(1-p)(E[s p(t)])^{2}}{(E[s(t)]+p E[s p(t)])^{2}}
$$

Mean service time and coefficient of variation are the two fundamental process parameters with regard to cycle time performance. ${ }^{[36]}$ The waiting time is directly proportional to the coefficient of variation. Hence, in order to improve the cycle time, the coefficient of variation of service times, i.e., the surplus time should be minimised. Reducing the process time variability is equivalent to increasing the system capacity when measured by cycle time response. ${ }^{[37]}$ The demand variability and process variability are buffered by "First In First Out" queue and buffer size (number of patients who can wait in a queue) can be estimated by using Equation 4.4.

Buffer size $=\frac{s(t)_{\max }-s(t)}{\operatorname{Takt}(t)} *$ Patient demand

Where $s(t)$ is the mean service time at the preceding department and $s(t)_{\max }$ is the maximum service time at the preceding department. Each department could have patients being serviced and waiting (buffers), i.e., WIP. When WIP is maximum, the demand in manufacturing plants could be blocked. However, when demand exceeds buffer capacity or departmental capacity in AEH, neither patient demand can be blocked (due to maximum WIP) nor can the patients be made to wait. Therefore, the total amount of work (patient demand) needs to be spread and synchronised along departments in the OPC. In order to balance the patient flow (line balancing), the relationship or the ratio of service times between the departments should also be considered in resource planning.

As discussed, there is a difference between forecasting and reality. When the OPC system is working according to a particular Takt, it should offer a margin for the operational problems 
that occur due to variability. ${ }^{[38]}$ Operator load (total work an employee can perform) varies according to the working speed of employees and thus depends on their skills. A realistic load should be given to the operator for a smooth and efficient flow without jeopardising the quality. This we take into account by multiplying Takt time by a factor called fudge factor or efficiency factor. Generally, an efficiency factor of $85-95 \%$ is used in designing industrial processes and this allows operators to work at a productive rate. This results in properly built quality products. ${ }^{[39-41]}$ In this case, it is estimated by coefficient of variation of the cycle time, $c(t)$.

$\mathrm{f}=\mathrm{CV}_{\mathrm{c}(\mathrm{t})}=\frac{\text { Standard deviation of } \mathrm{c}(\mathrm{t})}{\text { Mean of } \mathrm{c}(\mathrm{t})}$

If the OPC runs f\% lesser than the Takt time, it requires more resources but captures the operator load. If the OPC runs $\%$ more than Takt time, it captures constraints and allows buffers. Determining the Takt for a system is a design parameter that has to be assessed on the basis of the demand and service variability (buffer) and resource and space (layout) constraints. It is important for the OPC systems to consider the difference between Takt and target (throughput) as a waste and to improve it and not just accept it. Hence, the use of Takt time goes beyond the numerical calculations. The demand variability and process time variability, such as surplus and setup times, irregularities and fudge factor, help in setting the margin to keep the OPC working when the reality differs from what was planned.

We derive a new operations management where resources' planning is demand-driven and global for keeping all departments in pace, neither slow nor fast. Contrary to fast food chains, resources are scheduled here on the basis of the predicted demand considering short-term variability (nearly real-time) with a global perspective (not department-level but OPC-wide). We develop a mathematical model to identify the number of resources required and the parameters used in the process is listed in Table 4.1. The margin is a variable that is not directly used in the optimisation problem. Reaction time is an exogenous variable that is affected by the internal and external environment of the OPC. Therefore, its effect on the dependent variables is analysed in the simulation model.

The optimisation problem is to find the minimum number of resources to achieve the required Takt time. That is we have to find

$$
\operatorname{Min} \sum_{m=1}^{M} \sum_{n=1}^{N} z_{m} r_{m, n}
$$

Subject to the condition that 
Table 4.1: List of notations used in the mathematical model

\begin{tabular}{ll}
\hline Notation & Description \\
\hline$n$ & Number of departments in the OPC where $n=1, \ldots, N$ \\
$m$ & Number of time-slots in a day where $m=1, \ldots, M$ \\
$r_{m, n}$ & Number of resources in $m^{\text {th }}$ time-slot in $n^{\text {th }}$ department \\
$R_{\mathrm{T}}$ & Total number of available resources in the OPC \\
$f$ & Coefficient of variation of cycle time $c(t)$ to set the margin \\
Takt $(t)$ & Takt time \\
$z_{m}$ & Reciprocal of Takt time during $m^{\text {th }}$ time-slot, $z=1 /$ Tak $(t)_{m}(1+f)$ \\
$g_{n}$ & Ratio of average service time of $n^{\text {th }}$ department to average cycle time \\
$h_{n}$ & Ratio $s(t){ }_{n} / s(t)_{n+1}$ \\
$s(t)_{n}$ & Mean service time of the $n^{\text {th }}$ department \\
$p(t)_{n}$ & Processing time that includes service time and surplus time of $n^{\text {th }}$ department \\
$C_{p(t), n}^{2}$ & Squared coefficient of variation of processing time $P(t)$ of $n^{\text {th }}$ department \\
\hline
\end{tabular}

OPC: Outpatient clinic

$\sum_{m=1}^{M} \sum_{n=1}^{N} g_{n} r_{m, n} \leq R_{T}$

$\sum_{n=1}^{N} r_{n} \leq h_{n} r_{n+1}$

$\sum_{m=1}^{M} \frac{r_{m, n}}{s(t)_{n}\left(1+C_{p(t), n}^{2}\right)} \leq z_{m}$

$\sum_{n=1}^{N} r_{n} \leq R_{T}$

$r_{m, n} \geq 1$

$r_{m, n}$ integer

The objective (4.6) minimises the resources required by each department in each time-slot to achieve the required Takt time. Resources are the decision variables in this optimisation model. Constraints (4.7) and (4.8) deal with the identical service rate in all departments, which is neither too fast nor too slow, but in pace and within the total available resources (global optimisation). Constraint (4.9) relates service variability, cycle and Takt time to the resources. The solution is not just increasing the resources when departmental demand is high, or conversely, but doing so without disturbing the flow of the successive upstream or downstream departments. This synchronises the spread of work between the departments 
in the OPC. The constraint (4.10) shows that the number of resources in all departments in the $\mathrm{m}^{\text {th }}$ time-slot should be within an available total. This constraint takes care of the criterion of line balancing and buffers. Constraint (4.11) assures that at least one resource is always allocated to each department in all time-slots. Finally, constraint (4.12) requires the integer assignment of resources.

The mathematical model was solved using Integer Linear Programming (ILP). The ILP output was the resource plan that indicated the number of resources in each department in each of the time-slots. We explain the planning model with an example. We selected 11 time-slots of an hour each, patient demand was identified and Takt time was determined for each time-slot. The resources were scheduled based on this predictive plan for both the units as shown in Table 4.2. These resource plans were used in the OPC simulation model to observe their effects on performance measures. The time-slots with high patient demand were found to have smaller Takt time. This implies that the system needs to work faster for which the number of resources required must be scheduled to control waiting times. In the time-slots where the patient demand is less, the number of resources scheduled can be reduced to avoid under-utilisation.

Table 4.2: Output of the mathematical model compared to the existing resource schedule

\begin{tabular}{|c|c|c|c|c|c|c|c|c|c|}
\hline Time-slots & $\begin{array}{l}\text { Takt time } \\
\text { in min }\end{array}$ & NR & $\mathrm{RR}$ & Vision 1 & $\begin{array}{l}\text { Preliminary and } \\
\text { final exam } 1\end{array}$ & Refraction 1 & Tension 1 & Dilatation 1 & $\begin{array}{l}\text { Number of } \\
\text { resources in } \\
\text { unit } 1 / \text { both } \\
\text { units }\end{array}$ \\
\hline 1 & 0.8 & 2 & 2 & 1 & 4 & 4 & 2 & 1 & $16 / 28$ \\
\hline 2 & 1.2 & 2 & 2 & 1 & 4 & 4 & 2 & 1 & $16 / 28$ \\
\hline 3 & 0.6 & 3 & 3 & 2 & 4 & 4 & 2 & 1 & $20 / 32$ \\
\hline 4 & 0.7 & 2 & 3 & 1 & 4 & 4 & 2 & 1 & $17 / 29$ \\
\hline 5 & 0.7 & 2 & 3 & 1 & 4 & 4 & 2 & 1 & $17 / 29$ \\
\hline 6 & 1.2 & 1 & 2 & 1 & 3 & 4 & 1 & 1 & $13 / 23$ \\
\hline 7 & 1.4 & 1 & 2 & 1 & 3 & 3 & 1 & 1 & $12 / 21$ \\
\hline 8 & 2.4 & 1 & 1 & 1 & 2 & 2 & 1 & 1 & $9 / 16$ \\
\hline 9 & 5.4 & 1 & 1 & 1 & 1 & 1 & 1 & 1 & $7 / 12$ \\
\hline 10 & 12 & 1 & 1 & 1 & 1 & 1 & 1 & 1 & $7 / 12$ \\
\hline 11 & 20 & 1 & 1 & 1 & 1 & 1 & 1 & 1 & $7 / 12$ \\
\hline $\begin{array}{l}\text { Currently } \\
\text { used } \\
\text { schedule } \\
\text { for whole } \\
\text { day }\end{array}$ & - & 2 & 2 & 1 & 3 & 5 & 2 & 1 & $16 / 28$ \\
\hline
\end{tabular}

The OPC currently follows the resource schedule as shown in the last row of Table 4.2. As per current scheduling, the total number of resources scheduled in both units is 28 , only unit 1 and registration is 16 , and this remains the same for a month. OPC: Outpatient clinic 


\section{MATERIALS AND METHODS}

\section{Data Collection and Analysis}

Initially, the data was collected from the observations and interviews of patients and hospital staff (ophthalmologists, paramedical staff, managers and administrative officers) of AEH. Patient and process data were obtained from January 2012 to June 2012 from the in-house software, i.e., Integrated Hospital Management System (IHMS) and Clinical Management System (CMS). The data collected included the patient volume, arrival and exit times, service times, resource schedule, waiting and cycle times (including registration, service and waiting time in all departments) and the reaction time, obtained through interviews with the staff and managers. Data of 53802 patients were analysed and the data-fitting tool "Easy Fit" was used to determine the probability distribution of service and patient arrival time. The data analysis revealed that the patient arrival pattern had two peaks, at around 8:00 am and 10:00 am. Therefore, a bimodal Poisson distribution ${ }^{[42,43]}$ was selected to generate the arrival times (Equation 4.13).

$P=\left\{v_{1}, v_{2}\right\}$ and $\lambda=\left\{\lambda_{1}, \lambda_{2}\right\}$

Where $P$ is the sum of two Poisson distributions with mean arrivals $\lambda_{1}$ and $\lambda_{2}$ mixed with proportions $v_{1}=0.35$ and $v_{2}=0.65$. The goodness of fit test for input and output distribution was conducted using the Kolmogorov-Smirnov test. The managers of the OPC verified the workflow of the model using flowcharts and a structured walk-through.

\section{Model Development}

A discrete event simulation model of the OPC was developed using Java. A patient was the entity whose progress was tracked. Service times were uniformly distributed between the minimum and maximum service times from the empirical data of each department, which were randomly generated. The patient arrival time and the number of resources in each department were utilised from the empirical data. The managers of the OPC verified the program. Further, the simulation model was calibrated by assigning the reaction time randomly between 20-30 min to improve its accuracy. The simulation model was run with the empirical data and the performance parameters were collected. The results from the simulation model

Table 4.3: Validation of the simulation model with the existing OPC in AEH

\begin{tabular}{|c|c|c|c|c|c|c|}
\hline \multirow[t]{3}{*}{ Patient demand } & \multicolumn{3}{|c|}{ Waiting time in minutes } & \multicolumn{3}{|c|}{ Cycle time in minutes } \\
\hline & \multicolumn{2}{|c|}{ Mean $\pm S D$} & \multirow[t]{2}{*}{$P$ value } & \multicolumn{2}{|c|}{ Mean $\pm S D$} & \multirow[t]{2}{*}{$P$ value } \\
\hline & Existing AEH & Simulation model & & Existing $\mathrm{AEH}$ & Simulation model & \\
\hline Low & $48.6 \pm 12.45$ & $45.7 \pm 10.57$ & 0.5 & $98.9 \pm 14.25$ & $96.2 \pm 12.54$ & 0.4 \\
\hline Medium & $68.2 \pm 18.56$ & $66.1 \pm 19.11$ & 0.4 & $122.3 \pm 17.83$ & $119.9 \pm 20.43$ & 0.3 \\
\hline High & $82.1 \pm 25.02$ & $79 \pm 23.54$ & 0.6 & $138.9 \pm 27.12$ & $137.9 \pm 25.32$ & 0.5 \\
\hline
\end{tabular}

OPC: Outpatient clinic, AEH: Aravind Eye Hospital, SD: Standard deviation 
were compared with the empirical data of the OPC for validation as shown in Table 4.3 and no statistical difference was found between the two.

The difference in waiting and cycle times of the simulation model and the existing AEH procedure is due to the local optimisation performed by paramedical staff in their units. In the existing $\mathrm{AEH}$, whenever congestion is observed the paramedical staff manually changes the sequence for patients in RF and PE. However, in the simulation model, sequencing is performed for each patient.

\section{Experimental Design}

It is observed from the literature and the case study on AEH that the difference between planning and reality results in the operational problems. In this study, we measured waiting times and cycle times. This experimental design had three factors, namely, patient demand, scheduling rules (control of operations) and reaction time. Patient demand in $\mathrm{AEH}$ is huge and variable and affects the waiting times. In the literature, patient demand is found to be around 300 patients/week. Nevertheless, in AEH, the average demand is high, i.e., around 1800 patients/ day with $30.8 \%$ of the monthly patient demand being $1000-1600$ patients/day, $49.9 \%$ being 1600-2000 patients/day and $19.3 \%$ being greater than 2000 patients/day. Therefore, we classified patient demand into low, medium and high. The scheduling rules varied in two levels, namely, existing rules with fixed number of resources (local) and the rules as per the predictive resource plan with varying number of resources within a day (global). Since the reaction time affects the waiting times, we chose to experiment with different reaction times in minutes: $r$ (t1) that is $\leq 10,11 \leq r(t 2) \leq 20$ and $21 \leq r(t 3) \leq 30$. Reaction times were randomly assigned to the departments in the selected range. Additionally, to analyse the effect of reaction times on departmental performance, we selected six combinations of reaction times based on service times (high and low). There were in total $2^{1} \times 3^{1} \times 9^{1}=54$ experiments, and the performance measures were recorded for all experiments in the design. A full factorial experiment was carried out to estimate the effect of selected factors on performance parameters.

\section{Simulation Runs}

The experimental design was replicated 10 times with 540 runs to estimate the variability associated with the phenomenon. The simulation of a day took around 3-4 min per day. The seed in random variate was varied to generate different arrival times for the same mean patient arrivals. The same randomiser input was used for simulation, with two different scheduling scenarios, namely, existing (fixed) and predictive plan based (proposed). This assured that the results obtained were not due to randomness. The mean and standard deviations of the waiting and cycle times were collected. These results were compared with the existing $\mathrm{AEH}$. Analysis of variance (ANOVA) tests was conducted for statistical comparisons 
at a significance level of 0.05. Additionally, ANOVA tests were performed by using IBM SPSS to determine the significance of the main and interaction effects of predictive planning and reaction time on waiting and cycle times. Further, the proposed model was implemented in units 1 and 2 of the OPC. The daily resource schedule based on predictive planning was implemented for a month, the performance measures collected from the IHMS and CMS are presented in the results section.

\section{RESULTS}

\section{Simulation Results}

Given the inputs described in section 4.3, we report the results of the proposed predictive planning-based resource scheduling model, on two performance metrics, i.e., mean waiting time and mean cycle time. First, we present the results of the simulation experiments and later present the results of implementation. The analysis centred on the statistically significant trends for the performance measures. The mean cycle time for the existing scheduling scheme and predictive resource planning (simulation) was $120.1 \pm 19.7 \mathrm{~min}$ and $89.3 \pm 9.3 \mathrm{~min}$, respectively. The mean waiting time was $66.3 \pm 18.7 \mathrm{~min}$ and $37.5 \pm 8.9 \mathrm{~min}$, respectively. The mean and standard deviation of the waiting and cycle times for existing and predictive planning are compared in Tables 4.4 and 4.5 , respectively.

Average waiting times were reduced and regulated in all departments. The waiting times in both registration and preliminary and final examination departments were reduced significantly. At the same time, we notice that there is an increase in average waiting time in the vision department by a few minutes as shown in Figure 4.4. The figure also compares the waiting times in the departments for various combinations of different reaction times and their respective cycle times. Reaction times were selected on the basis of low and high service times. Example for mix1: Departments with low (L) service times $(<5 \mathrm{~min})$ is $\mathrm{r}(\mathrm{t} 1)$ and high $(\mathrm{H})$ service times is $r(\mathrm{t} 2)$.

Reaction time is an exogenous variable that influences performance measures. The two-way ANOVA-tests $(P=0.05)$ showed the significance of the main and interaction effects of predictive planning and the reaction time is presented in Table 4.6.

Planning has a significant main effect on waiting times, whereas the main effect of reaction time is not significant; however, the interaction effects of planning and reaction time are significant. Further, pair-wise comparisons were performed for the three levels of reaction times: $r(\mathrm{t} 1)-r(\mathrm{t} 2), r(\mathrm{t} 2)-r(\mathrm{t} 3)$ and $r(\mathrm{t} 1)-r(\mathrm{t} 3)$ and their significance were $0.205,0.226$ and 0.023 , respectively. The interaction effect of $r(t 1)$ and $r(t 2)$ was comparatively more than $r(t 3)$ 
(Figure 4.5). The results show that the reaction time contributes around 5\% (4.89\%) in reducing waiting times. As the reaction times between departments varied, tests of betweensubjects effects were conducted. Main and interaction effects of departments, planning

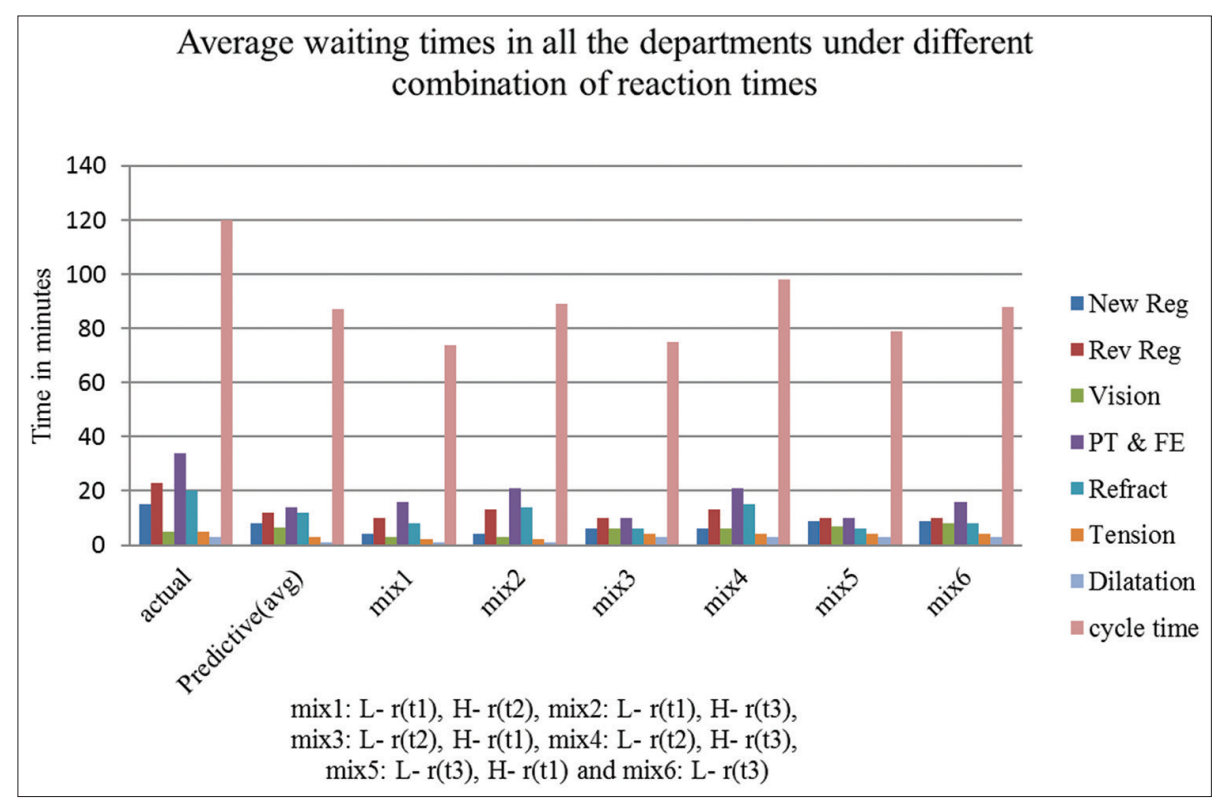

Figure 4.4: Effect of reaction times on average waiting times in all departments and average cycle time

Table 4.4: Comparison of average waiting time in minutes of existing and predictive plan based scheduling

\begin{tabular}{lccccccc}
\hline Patient demand/reaction time $r(t)$ & \multicolumn{2}{c}{$\begin{array}{c}\text { Existing scheduling with local control } \\
\text { of operations }\end{array}$} & & \multicolumn{3}{c}{$\begin{array}{c}\text { Predictive plan based scheduling } \\
\text { with global control of operations }\end{array}$} \\
\cline { 2 - 3 } & \multicolumn{3}{c}{ Mean \pm SD } & & & Mean $\pm S D$ \\
\cline { 2 - 3 } & $r(t 1) \min$ & $r(\mathrm{t} 2) \min$ & $r(t 3) \min$ & & $r(t 1) \min$ & $r(t 2) \min$ & $r(t 3) \min$ \\
\hline Low & $44.3 \pm 8.3$ & $49.3 \pm 8.6$ & $52.1 \pm 10.9$ & & $15.1 \pm 6.7$ & $32.6 \pm 8.1$ & $44.7 \pm 9.2$ \\
Medium & $56.6 \pm 15.8$ & $62.9 \pm 15.7$ & $71.8 \pm 18.2$ & & $24.1 \pm 7.3$ & $36.2 \pm 9.2$ & $52.9 \pm 10.9$ \\
High & $69.5 \pm 20.1$ & $75.3 \pm 21.9$ & $83.1 \pm 23.9$ & & $30.1 \pm 7.8$ & $44.6 \pm 9.9$ & $54.2 \pm 11.1$ \\
\hline
\end{tabular}

OPC: Outpatient clinic, SD: Standard deviation

Table 4.5: Comparison of average cycle time in minutes of existing and predictive plan based scheduling

\begin{tabular}{|c|c|c|c|c|c|c|}
\hline \multirow[t]{3}{*}{$\begin{array}{l}\text { Patient demand/reaction } \\
\text { time } r(t)\end{array}$} & \multicolumn{3}{|c|}{$\begin{array}{c}\text { Existing scheduling with local control of } \\
\text { operations }\end{array}$} & \multicolumn{3}{|c|}{$\begin{array}{l}\text { Predictive plan based scheduling with } \\
\text { global control of operations }\end{array}$} \\
\hline & \multicolumn{3}{|c|}{ Mean $\pm S D$} & \multicolumn{3}{|c|}{ Mean $\pm S D$} \\
\hline & $r(t 1) \min$ & $r(t 2) \min$ & $r(t 3) \min$ & $r(t 1) \min$ & $r(t 2) \min$ & $r(t 3) \min$ \\
\hline Low & $93.5 \pm 8.4$ & $99.6 \pm 8.8$ & $101.1 \pm 10.8$ & $70.3 \pm 6.9$ & $85.8 \pm 8$ & $101.6 \pm 9.3$ \\
\hline Medium & $107.5 \pm 16.1$ & $112.3 \pm 16.9$ & $119.9 \pm 19.9$ & $74.8 \pm 7.7$ & $86.4 \pm 9.7$ & $104.8 \pm 12.3$ \\
\hline High & $119.7 \pm 20.9$ & $121.1 \pm 22.1$ & $132.7 \pm 24.5$ & $78.2 \pm 7.5$ & $94.5 \pm 10.2$ & $107.1 \pm 12.4$ \\
\hline
\end{tabular}

SD: Standard deviation 
Table 4.6: Tests of between-subjects effects

\begin{tabular}{lccccc}
\hline Source & Type III sum of squares & df & Mean square & F & Significance \\
\hline Intercept & 829177.965 & 1 & 829177.965 & 46.410 & 0.021 \\
Planning & 52486.767 & 1 & 52486.767 & 216.089 & 0.005 \\
Reaction time & 35732.657 & 2 & 17866.328 & 73.556 & 0.068 \\
Planning*Reaction time & 485.788 & 2 & 242.894 & 3.274 & 0.049 \\
\hline
\end{tabular}

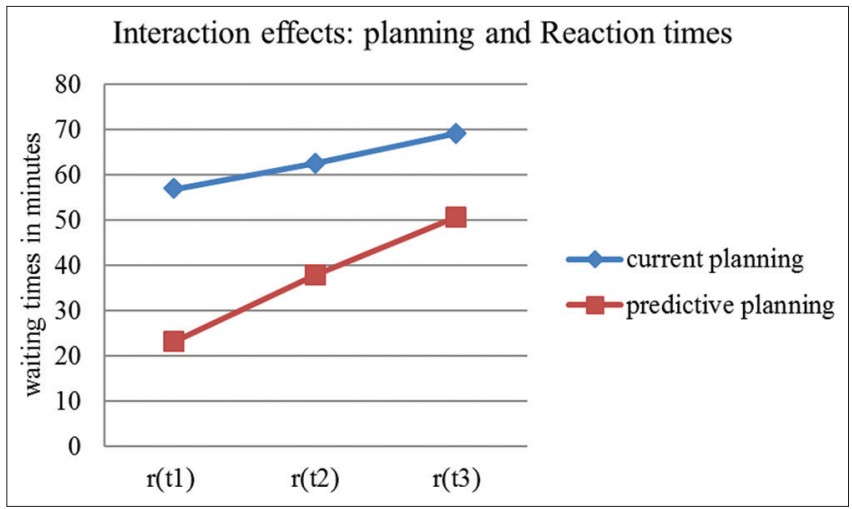

Figure 4.5: Result of two-way ANOVA test

and reaction time on performance measures were analysed (with $P$-values for department * planning $(0.001)$, department * reaction time $(0.50)$ and planning * reaction time $(0.003)$. We observe that main and interaction effects of planning are significant in all departments; whereas, the reaction time is not significant in the departments with shorter service times.

\section{Implementation Results}

The predictive resource planning was implemented during January 2014. The performance measures were collected from IHMS and CMS. The mean cycle and waiting times after implementation were $92.4 \pm 9.1 \mathrm{~min}$ and $39.0 \pm 8.2 \mathrm{~min}$, respectively (Table 4.7). The existing resource plan had a fixed number (28) of resources in both units in a day; whereas, the predictive resource plan had a minimum of 12 to a maximum of around 30-34 resources. The additional resources were scheduled from different clinics of $A E H$ and the resources reduced during some time-slots were utilised for maintaining patient records (back-end work). As seen in Table 4.2, when compared to the existing schedule, the number of resources required is higher during time-slots 3 and 4 and is lower in a few time-slots.

The performance measures of the proposed model were significantly different from those of the existing situation. A $P$-value of 0.05 was selected, which means that the null hypothesis is rejected for a $P<0.05$ and the difference is statistically significant. 
Table 4.7: Average waiting times and cycle times before and after implementation of the predictive resource planning in the OPC

\begin{tabular}{|c|c|c|c|c|c|c|c|c|c|}
\hline \multirow[t]{3}{*}{ Patient demand } & \multicolumn{3}{|c|}{ Number of resources } & \multicolumn{3}{|c|}{ Waiting time in minutes } & \multicolumn{3}{|c|}{ Cycle time in minutes } \\
\hline & \multirow[t]{2}{*}{ Before } & \multicolumn{2}{|c|}{ After } & \multirow{2}{*}{$\frac{\text { Before }}{\text { Mean } \pm S D}$} & \multirow{2}{*}{$\frac{\text { After }}{\text { Mean } \pm S D}$} & \multirow[t]{2}{*}{$P$ value } & \multirow{2}{*}{$\begin{array}{c}\text { Before } \\
\text { Mean } \pm S D\end{array}$} & \multirow{2}{*}{$\frac{\text { After }}{\text { Mean } \pm S D}$} & \multirow[t]{2}{*}{$P$ value } \\
\hline & & Min & Max & & & & & & \\
\hline Low & 28 & 12 & 30 & $48.6 \pm 12.5$ & $34.3 \pm 7.1$ & 0.03 & $96.0 \pm 14.3$ & $85.8 \pm 7.7$ & 0.04 \\
\hline Medium & 28 & 12 & 32 & $68.2 \pm 18.6$ & $39.3 \pm 8.4$ & 0.01 & $118.3 \pm 17.8$ & $92.4 \pm 8.9$ & 0.02 \\
\hline High & 28 & 12 & 34 & $82.1 \pm 25.0$ & $43.4 \pm 9.1$ & 0.01 & $136.0 \pm 27.1$ & $99.1 \pm 10.6$ & 0.02 \\
\hline
\end{tabular}

OPC: Outpatient clinic, SD: Standard deviation

\section{DISCUSSION}

OPC system in AEH consists of different departments that schedule their resources ahead of time (once a month). The OPC which is an open-loop system is prone to variability, and the current method of planning caused the formation of bottlenecks in a few departments and resulted in prolonged waiting and cycle times. The OPC in AEH, like other hospitals (as seen in literature), managed its services by locally planning resources on the basis of the average demand (day-wise) and not the actual demand and its variability.

In this study, resources are planned and scheduled in the global perspective on the basis of actual demand (near to real-time) and by incorporating short-term variability. The findings of the study confirm that the predictive planning model reduces the average waiting time by $43.4 \%$ during simulation (from $66.3 \mathrm{~min}$ to $37.5 \mathrm{~min}$ ) and by $41.1 \%$ during its implementation (from $66.3 \mathrm{~min}$ to $39.0 \mathrm{~min}$ ) in the OPC. In addition, the standard deviations of waiting times were reduced significantly. The study demonstrates the effect of patient demand, planning and scheduling rules, control of operations and reaction times on waiting and cycle times (Table 4.5). It is found that global control of operations for planning and scheduling resources in departments improves waiting times when compared to the local control of operations.

Besides scheduling rules and control of operations, the reaction time, an exogenous variable, also influences the waiting time to some extent. ANOVA was used to determine the main and interaction effects of predictive planning and reaction time. Effects of predictive planning on performance measures are significant in all departments. Improvement in reaction times cannot influence waiting times on its own but has a significant effect on waiting times and cycle times when used along with predictive planning (Table 4.6). The reaction time varies among departments and the reaction time of the departments that have longer service times has a significant influence on waiting and cycle times (Figure 4.4). The influence of reaction time on the performance measures is of greater importance when the patient demand is high and being prepared for variability improves waiting times. 
Waiting times have increased in some departments like vision by a few minutes. This is because of the constraint (4.7) that balances the patient flow within the available resources. The number of resources required to achieve a particular Takt time may be high; however, due to resource and flow balance constraints (an equal spread of work), the scheduling model reduces the number of resources in few departments. As a consequence, waiting times in few of these departments have increased (Figure 4.4).

The resource schedule obtained by the proposed model shows that a different number of resources is scheduled during different times of the day (Table 4.2). When the patient demand is varying, the service rate should not remain constant. Hence, OPCs need to plan their resources according to the demand for which they should have a flexibility of resources. This predictive planning model considers the variability in demand in the short-term (hour by hour) and makes planning better and nearer to real-time. The current planning model estimates the total amount of work in a day and adapts resources accordingly. Planning is a decision taken much ahead of time and when the reality is different from planning, departments optimise locally which results in sub-optimisation. The predictive resource planning considers real-time operational problems that occur due to variability and uncertainty during the planning stage. Additionally, the model helps the management to identify resource requirements, i.e., types and quantities of resources to be prepared for the growth and expansion of future demand.

Takt time management synchronises the total amount and spread of work. It has been observed that it is applied to systems with even and stable demand, for longer time horizons and with a global perspective. However, in this study, Takt time management has been applied to uneven and unstable demand (all walk-in patients), in a shorter time horizon. As there is no control on patient demand, it is much higher than the maximum WIP the OPC can manage, and the patient demand cannot be blocked as done in a manufacturing system. Therefore, setting the Takt over a shorter period captured variability in the short-term ( $1 \mathrm{~h}$ ) and resources were scheduled every hour. The variability and uncertainty are inherent to OPC systems and cannot be eliminated. Nevertheless, they can be handled better. Using Takt time in resource planning brings predictability into the design effort and eliminates unplanned overtime work of resources. This implies that variability needs to be accounted for during the planning stage for real-time workflow optimisation. It balances the patient flow by matching the supply in accordance with the patient demand. This study demonstrates that Takt time management can also be applied to open loop systems where variability is high.

The literature shows that patient demand has been matched with resources, but planning often considered forecasting only over a long period. Planning often failed to incorporate variability in the short-term, which increased the gap between planning and reality. In this study, the predictive resource planning model integrates stochasticity in patient demand 
throughout the day and matches the resources accordingly in the short-term. It also incorporates the interdependencies between departments and generates a plan that optimises resources in the OPC perspective (global).

Some factors caused the implementation results to be different from what could be expected on the basis of the simulation study. Although the resources were scheduled on the basis of the predictive planning model, they sometimes arrived late to the departments as they were shared among other clinics in AEH. The predictive planning was implemented in only two units of the OPC and other clinics followed their regular scheduling rules. The planning model considered the constraints on resources and space/layout but did not consider resource sharing. The late arrival of resources and non-uniform patient arrivals or patient distribution within the time-slots affect the waiting times. Sometimes, more patient arrivals at the end of a time-slot increase the waiting time.

Healthcare settings like OPCs or hospitals vary in terms of complexity, patient groups and processes. Therefore, requirements for planning might vary. Many processes and arrivals are not deterministic and need to be optimised in the short-term through advanced planning systems. How we use this planning system might not only depend on the algorithms but also on other factors, such as the organisation of hospital/OPC, cross-skills of the staff (so that they can be transferred between departments) and the layout of the building. As a part of future work, we intend to analyse how Takt time management could influence resource utilisation and how to extend this planning model and incorporate resource sharing.

\section{CONCLUSION}

This study analyses the role played by predictive resource planning (near to real-time) in improving waiting and cycle times in OPCs. The study integrates two facets of planning, namely, demand-driven and global perspective. The resource planning is based on the actual (near to real-time) demand. Short-term variability in demand should be incorporated to make planning better and nearer to reality. Planning with a global perspective overcomes the problem of unregulated waiting times, which means that departments work neither too fast not too slow. Predictive resource planning model utilised Takt time management to set pace between demand and service and balance patient flow by global optimisation.

Resource planning is especially important for the operations management of open loop systems like OPCs as waiting time is a major concern for quality care. The variability and uncertainty in OPC systems can be minimised (nearly closed loop) by incorporating patient volume, patient types, resources and reaction times in real-time optimisation, thus improving the waiting and cycle times. Although this reaction time is an exogenous variable, the predictive 
resource planning model helps OPCs to avoid longer reaction times as the demand-driven resource schedule is known in advance. It was observed from this study that the model made a positive impact on some of the aforementioned drawbacks even with reaction times as long as $30 \mathrm{~min}$. However, the model performs better with shorter reaction times. As the model was robust, small drawbacks in implementation did not alter the outcomes. This planning model has been implemented in an eye care OPC and can be extended to general hospitals by considering their demand forecast, precedence constraints and workflow complexities.

\section{REFERENCES}

1. Huang XM. Patient attitude towards waiting in an outpatient clinic and its applications. Health Serv Manag Res Off J Assoc Univ Programs Health Adm HSMC AUPHA 1994;7(1):2-8.

2. Pillay DI, Ghazali RJ, Manaf NH, Abdullah AH, Bakar AA, Salikin F, et al. Hospital waiting time: The forgotten premise of healthcare service delivery? Int J Health Care Qual Assur 2011;24(7):506-22.

3. Zhu Z, Heng BH, Teow KL. Analysis of factors causing long patient waiting time and clinic overtime in outpatient clinics. J Med Syst 2012;36(2):707-13.

4. Harper PR. A framework for operational modelling of hospital resources. Health Care Manag Sci 2002;5(3):165-73.

5. Hassan MH, Zaghloul AA, Mokhtar SA. The probability distribution of attendance to hospital emergency units for school students in Alexandria. J Egypt Public Health Assoc 2005;80(1-2):127-51.

6. Nguyen TB, Sivakumar Al, Graves SC. A network flow approach for tactical resource planning in outpatient clinics. Health Care Manag Sci 2015;18(2):124-36.

7. Vermeulen IB, Bohte SM, Elkhuizen SG, Lameris H, Bakker PJ, Poutre HL. Adaptive resource allocation for efficient patient scheduling. Artif Intell Med 2009;46(1):67-80.

8. Vissers JM, Bertrand JW, de Vries G. A framework for production control in health care organizations. Prod Plann Contr Manag Oper 2001;12(6):591-604.

9. Mansdorf BD. Allocation of resources for ambulatory care -A staffing model for outpatient clinics. Public Health Rep 1975;90(5):393-401.

10. Yurko LC, Coffee TL, Fusilero J, Yowler CJ, Brandt CP, Fratianne RB. Management of an inpatient-outpatient clinic an eight-year review. J Burn Care Rehabil 2001;22(3):250-4.

11. Rouppe van der Voort MM, van Merode FG, Berden BH. Making sense of delays in outpatient specialty care: A system perspective. Health Policy 2010;97(1):44-52.

12. Edward GM, Das SF, Elkhuizen SG, Bakker PJ, Hontelez JA, Hollmann MW, et al. Simulation to analyse planning difficulties at the preoperative assessment clinic. Br J Anaesth 2008;100(2):195-202.

13. Molema JJ, Groothuis S, Baars IJ, Kleinschiphorst M, Leers EG, Hasman A, et al. Healthcare system design and parttime working doctors. Health Care Manag Sci 2007;10(4):365-71.

14. Ludwig M, Van Merode F, Groot W. Principal agent relationships and the efficiency of hospitals. Eur J Health Econ 2010;11(3):291-304.

15. van Merode GG, Groothuis S, Hasman A. Enterprise resource planning for hospitals. Int J Med Inform 2004;73(6):493-501.

16. McKee M, Waghorn A. Why is it so difficult to organise an outpatient clinic? J Health Serv Res Policy 2000;5(3):140-7.

17. Hopp WJ, Spearman ML. Factory Physics: Foundations of Manufacturing Management. New York, NY: Irwin, McGraw-Hill; 2001. p. 698.

18. Chan H, Lo S, Lee L, Lo W, Yu W, Wu Y, et al. Lean techniques for the improvement of patients' flow in emergency department. World J Emerg Med 2014;5(1):24-8.

19. Hopp WJ, Lovejoy WS. Hospital Operations: Principles of High Efficiency Health Care. New Jersey: (FT Press 
Operations Management), FT Press; 2012. p. 623.

20. Liker JK. The Toyota Way: 14 Management Principles from the World's Greatest Manufacturer. New York: McGraw-Hill; 2004. p. 330.

21. dos Reis Alvarez R, Antunes Jr JA. Takt-time: Concepts and context in Toyota Production System. Gestão Produção 2001;8(1):1-18.

22. Day RW, Dean MD. Robert garfinkel and steven thompson, improving patient flow in a hospital through dynamic allocation of cardiac diagnostic testing time-slots. Decis Support Syst 2010;49:463-73.

23. Eswaramoorthi M, Kathiresan GR, Jayasudhan TJ, Prasad PS, Mohanram PV. Flow index based line balancing: A tool to improve the leanness of assembly line design. Int J Prod Res 2012;50(12):3345-58.

24. Sandanayake YG, Oduoza CF. Dynamic simulation for performance optimization in just-in-time-enabled manufacturing processes. Int J Adv Manuf Technol 2009;42(3-4):372-80.

25. Chaudhary B, Modi AG, Reddy K. Right to sight: A management case study on Aravind Eye Hospitals. Zenith Int J Multidiscip Res 2012;2(1):447-57.

26. Rangan VK, Thulasiraj RD. Making sight affordable (innovations case narrative: The Aravind eye care system). Innov Technol Governance Global Fall 2007;2(4):35-49.

27. Andersen MM, Poulfelt F. Beyond Strategy: The Impact of Next Generation Companies. New York: Routledge; 2014.

28. Natchiar G, Thulasiraj R, Sundaram RM. Cataract surgery at Aravind Eye Hospitals: 1988-2008. Community Eye Health 2008;21(67):40-2.

29. Cayirli T, Veral E. Outpatient scheduling in health care: A review of literature. Prod Oper Manag 2003;12(4):519-49.

30. Gupta D, Denton B. Appointment scheduling in health care: Challenges and opportunities. IIE Trans 2008;40:800-19.

31. Brilliant L, Brilliant G. Aravind: Partner and social science innovator (innovations case discussion: Aravind eye care system). Innov Technol Governance Global 2007;2(4):50-2.

32. Mehta PK, Shenoy S. Infinite Vision: How Aravind Became The Greatest Business Case for Compassion. San Franciso: Berrett-Koehler Inc.; 2011. p. 336.

33. Aravind Eye Care System: Activity Report. Madurai; 2014-15. p. 76.

34. Smith JM, Tan B. Handbook of stochastic models and analysis of manufacturing system operations. International Series in Operations Research and Management Science. Vol. 192. New York: Springer-Verlag; 2013. p. 373.

35. Hopp WJ, Spearman ML. Factory Physics: Foundations of Manufacturing Management. New York, NY: Irwin, The McGraw-Hill; 1996. p. 668.

36. Jacobs JH, Etman LF, van Campen EJ, Rooda JE. Characterization of operational time variability using effective process times. IEEE Trans Semicond Manuf 2003;16(3):511-20.

37. Curry GL, Feldman RM. In: Manufacturing Systems Modeling and Analysis. Berlin Heidelberg: Springer-Verlag; 2010. p. 109-23.

38. Rother M. Toyota Kata: Managing People for Improvement, Adaptiveness and Superior Results. San Francisco, CA: McGraw-Hill Professional; 2009. p. 306.

39. Fekete M, Hulvej J. "Humanizing" takt time and productivity in the labor-intensive manufacturing systems. In: Knowledge Management and Innovation Management, Knowledge and Learning International Conference, Croatia; 2013.

40. Ortiz CA. Kaizen Assembly: Designing, Constructing and Managing a Lean Assembly Line. Boca Raton, FL: CRC Press; 2006. p. 260.

41. Duggan KJ. Creating Mixed Model Value Steams-Practical Lean Techniques for Building to Demand. USA: Productivity Press; 2002. p. 224.

42. Li J, Zha H. Two-way poisson mixture models for simultaneous document classification and word clustering. Comput Stat Data Anal 2006;50(1):163-80.

43. Karlis D, Xekalaki E. Mixed poisson distributions. Int Stat Rev 2005;73:35-58. 


\section{Integral patient scheduling to reduce patient waiting times in outpatient clinics

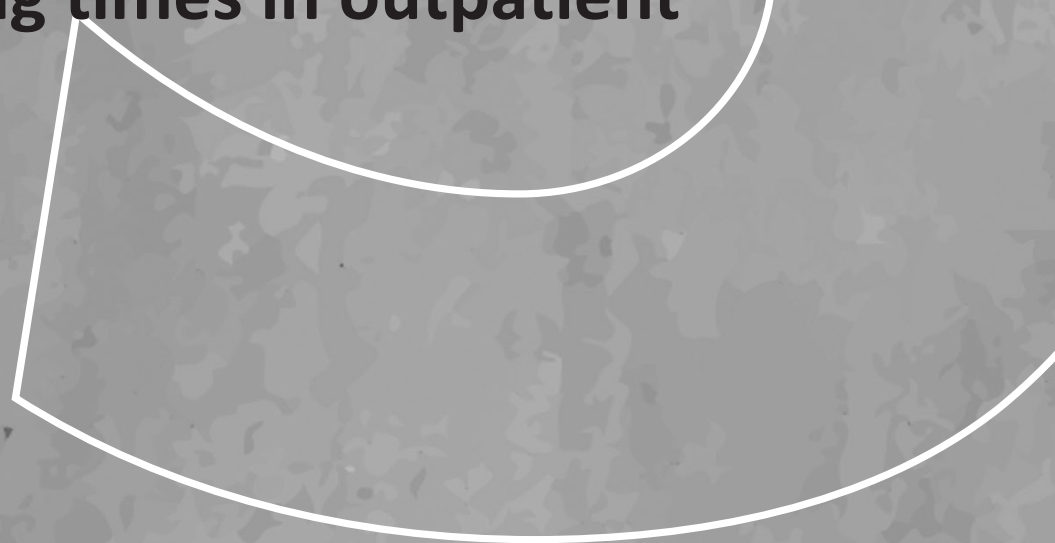

Submitted for publication:

Munavalli JR, Rao SV, Srinivasan A, van Merode GG. 



\section{INTRODUCTION}

Patient scheduling has become an important aspect in operations management of outpatient clinics (OPCs). In most developing countries, studies have shown that patients spend a long time in the OPCs before seeing a doctor. ${ }^{[1]}$ These delays in care are detrimental not only due to the costs incurred but also in terms of patient satisfaction and adverse outcomes. ${ }^{[2]}$ Providing high-quality services with limited resources places greater emphasis on the need for efficient utilisation of resources. An important operational issue in health care delivery thereby involves increasing utilisation and access by minimising delays in care delivery.

In general, OPCs schedule patients depending on the availability of resources (doctors and equipment) with the goal of matching demand with supply, most often to maximise resource utilisation and profit. Previous studies on patient scheduling have discussed various methods for scheduling appointments. ${ }^{[3-6]}$ In the literature, we notice that appointment scheduling has evolved from manual methods to automated methods. ${ }^{[7-10]}$ Different factors that can potentially increase the unpredictability in OPCs, such as no-shows and double booking, have been incorporated to optimise hospital performance ${ }^{\left[{ }^{8]}\right.}$ Along with scheduled patients, OPCs also get walk-in patients; therefore, OPCs reserve few time-slots for walk-in patients. ${ }^{[11-16]}$ However, OPCs being open-loop systems are affected by their environment and experience variability and uncertainties that are caused by unpredictable clinical pathways, patient arrivals (early, late or random) and varied service times. Patients, whether scheduled or walk-in, wait for care.

A patient pathway is a route taken by a patient from his/her first contact or registration until the diagnosis or completion of treatment. Most of the processes in an OPC have to be carried out in sequence as they depend on the outcomes of the previous steps. With increased complexity in diagnoses and treatment, patients have to take complex pathways and visit multiple departments for various tests or evaluations. Time spent in each department by each patient may vary significantly. ${ }^{[17,18]}$ The departments often manage and control their resources, patients and activities independently. In order to achieve efficiency, operations are most often optimised locally or departmentally without coordination from other departments, because of which patients wait in some departments and resources wait in some other departments. Since OPCs are functionally organised, the status of downstream departments is often not known to upstream departments. The department-centric scheduling (local optimisation) might improve departmental efficiency (waiting times), but does not necessarily improve OPC-wide efficiency (cycle times). ${ }^{[19]}$

During registration, complete patient pathways or the actual status of the departments is not always known because of which patients wait at various departments. Essentially, patient pathways depend on the type of clinic, type of disease, type of treatment and type of patient. 
For example, a general OPC may have more complex pathways than a speciality clinic. Patient scheduling approaches that are commonly used are inadequate as they are not based on the actual status of OPC system (departments and demand) and lack path optimisation (global). Therefore, there is a need to design an OPC system that schedules patients based on the actual status of OPC (pull system) rather than pre-scheduling patients through an appointment system (push system). It means that an OPC system should optimise patient pathways based on actual status so that the waiting time is minimised. We propose an integral patient scheduling method that integrates both actual status and pathway optimisation through global scheduling. For this, there is a need to collect the OPC system status and analyse the optimal patient path in real-time.

In recent years, bio-inspired computing algorithms like Ant Colony Optimisation (based on the study of ants) have been used to solve such optimisation problems. ${ }^{[20,21]}$ These are used to find the shortest path between a source and its destination. Ants in the natural world wander randomly in search of food and leave a trail of pheromones while returning to their colonies after finding food. Other ants follow this trail of pheromones to find the food. Over a period, the shortest path will have the highest concentration of pheromones. However, the pheromone trail starts evaporating with time. This makes the ants search for alternative and sometimes better paths, thereby improving routing, congestion control, cost and time. ${ }^{[22-29]}$

The OPC in Aravind Eye Hospital (AEH) was a good test case for this study, as it fits in the assumed working conditions. AEH resembles an assembly line system (a line of workers and equipment along which a product being assembled passes consecutively from one operation to the other until completion). ${ }^{[30-33]}$ However, some aspects of the OPC differ from assembly line systems like local control of operations in departments and uneven patient demand. The patient arrivals are random (no appointment system is used) and independent. This makes patient demand highly variable and uncertain. The OPC provides care for all the patients on the same day (Rule 1). We present the patient scheduling in the OPC of AEH in the next section and then state the hypothesis of this study.

\section{Patient Scheduling in the OPC of AEH}

AEH is a renowned eye care hospital in Madurai, South India that provides patient-centric care. ${ }^{[30,34,35]}$ AEH has performed 401,529 surgeries and treated 2,396,864 outpatients during 2014-2015. ${ }^{[36]}$ The hospital functions with assembly line efficiency, strict quality norms, process standardisation, cost control and above all, high patient volumes. The OPC in AEH is open from 7 am to $6 \mathrm{pm}$. It consists of two identical units (with the same number of resources and equipment). The OPC has its in-house software, namely, integrated hospital management system (IHMS) and clinical management system (CMS) that records process and patient information. The managers of AEH manage the departments in the OPC locally. The functional 
and operational structure of the OPC in AEH with respect to patient scheduling is shown in Figure 5.1. Departments, such as new registration (NR) and review registration (RR), are common to the two units of the OPC and each unit has five departments, namely, vision (V), refraction (RF), tension (TN), dilatation (DL) and preliminary and final examination (PE and $F E$ ).

AEH follows several kinds of rules that are briefly described in Box 5.1. All the queues are on the first-come first-served basis (Rule 2). The patient flow arrows in Figure 5.1 show the possible pathways for new and review patients. The patient pathway starts with registration and finishes with a FE. A patient moves through various departments, and the pathways for new and review patients differ. Around $5 \%$ of the total number of patients exit after the PE. The order of departments RF and PE can be interchanged in the pathway. The IHMS

Box 5.1: Rules in the OPC in AEH

Rule 1: All patients are provided with service/care on the same day of arrival.

Rule 2: All the queues are FCFS.

Rule 3: Patients are scheduled to units 1 and 2 alternately.

Rule 4: Load distribution in both units is same.

Rule 5: Number of resources is fixed throughout the month.

Rule 6: Number of resources in unit 1 = number of resources in unit 2.

Rule 7: Patients must complete RF and PE before TN.

Rule 8: Control of operations is local (department-centric).

FCFS: First come first serve, OPC: Outpatient clinic, AEH: Aravind Eye Hospital, RF: Refraction,

PE: Preliminary examination, TN: Tension

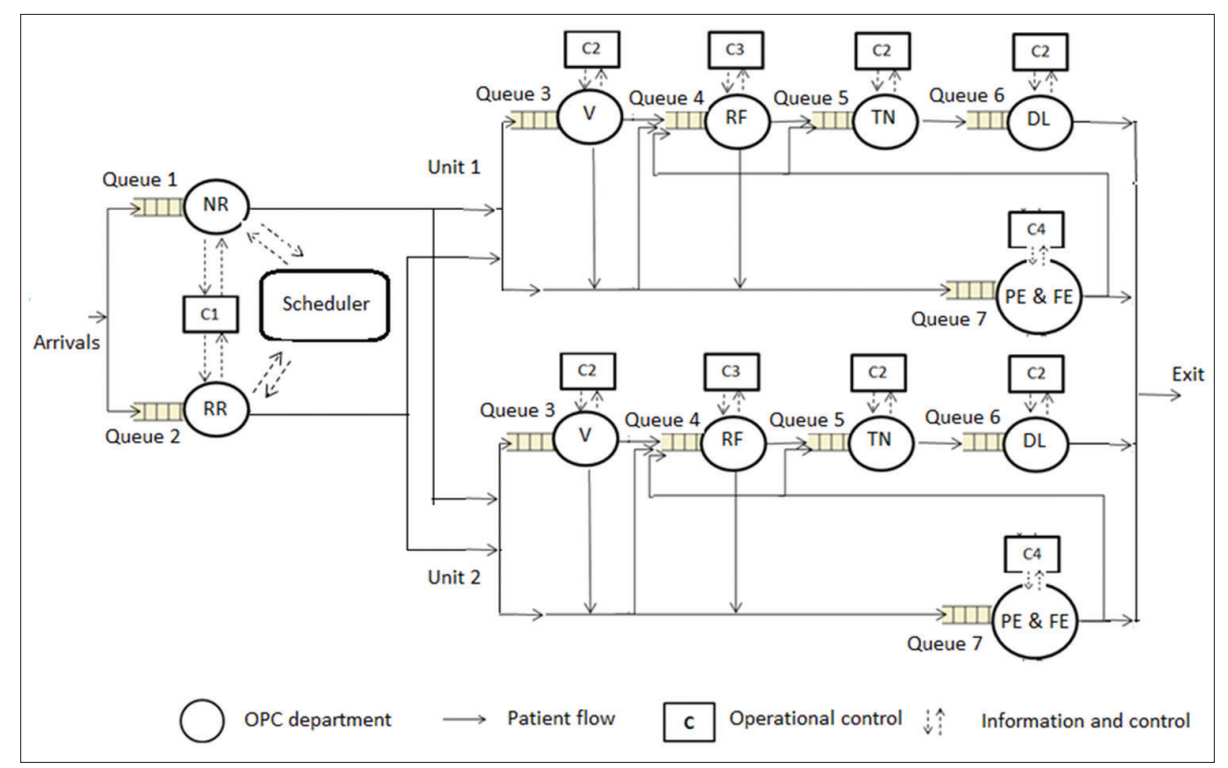

Figure 5.1: Functional and operational structure in the outpatient clinic system of Aravind Eye Hospital 
schedules patients alternatively to either of the units (Unit 1 and 2) during registration (Rule 3). The OPC follows an equal load distribution (equal number of patients serviced) between the two units (Rule 4). The controllers (C) in Figure 5.1 are the managers who plan, schedule and control activities in the departments. Operations are managed locally in the departments despite a limited knowledge about the downstream departments. Managers manually schedule patients to the preliminary test or refraction test, depending on the queues in the two departments.

Although the OPC in AEH is efficient, it still faces operational problems like long waiting times and cycle times. The OPC, like other hospitals (see Chapter 2, literature review), is an open loop system with uneven demand, lacks synchronisation between departments and does not schedule patients on the basis of the actual status of the OPC system. Due to the lack of path optimisation, patients wait in overcrowded departments at times and resources remain idle in other departments. In order to improve its operations, the OPC needs to schedule patients in real-time by determining the pathways on the basis of the actual status of all departments in the OPC. Therefore, we develop an integral patient scheduling system, which schedules patients to the pathway that has minimum waiting time. Further, hybrid ant agent algorithm is developed for integral patient scheduling.

We state the hypothesis of this study:

"An OPC system with disparate subsystems can reduce cycle/waiting time by global optimisation, using integral patient scheduling."

The remainder of this paper is organised as follows: In Section 5.2, we present an integral patient scheduling model and describe a hybrid ant agent algorithm. In Section 5.3, we describe materials and methods that include data collection, model development, experimental design, simulation study and implementation of the scheduling model in the OPC. In Section 5.4, we report the results from different scenarios created with real case studies. Then, we discuss our findings in Section 5.5 and conclusions in Section 5.6.

\section{INTEGRAL PATIENT SCHEDULING MODEL}

The integral patient scheduling model aims to schedule patients to the pathways that are determined by using the actual status of departments for minimising waiting times. The integral patient scheduling model uses three levels of control (Figure 5.2).

Patient arrival at registration triggers the scheduling model. At the first level, we collect patient data, process data from all departments and define the possible patient pathways in the OPC. 


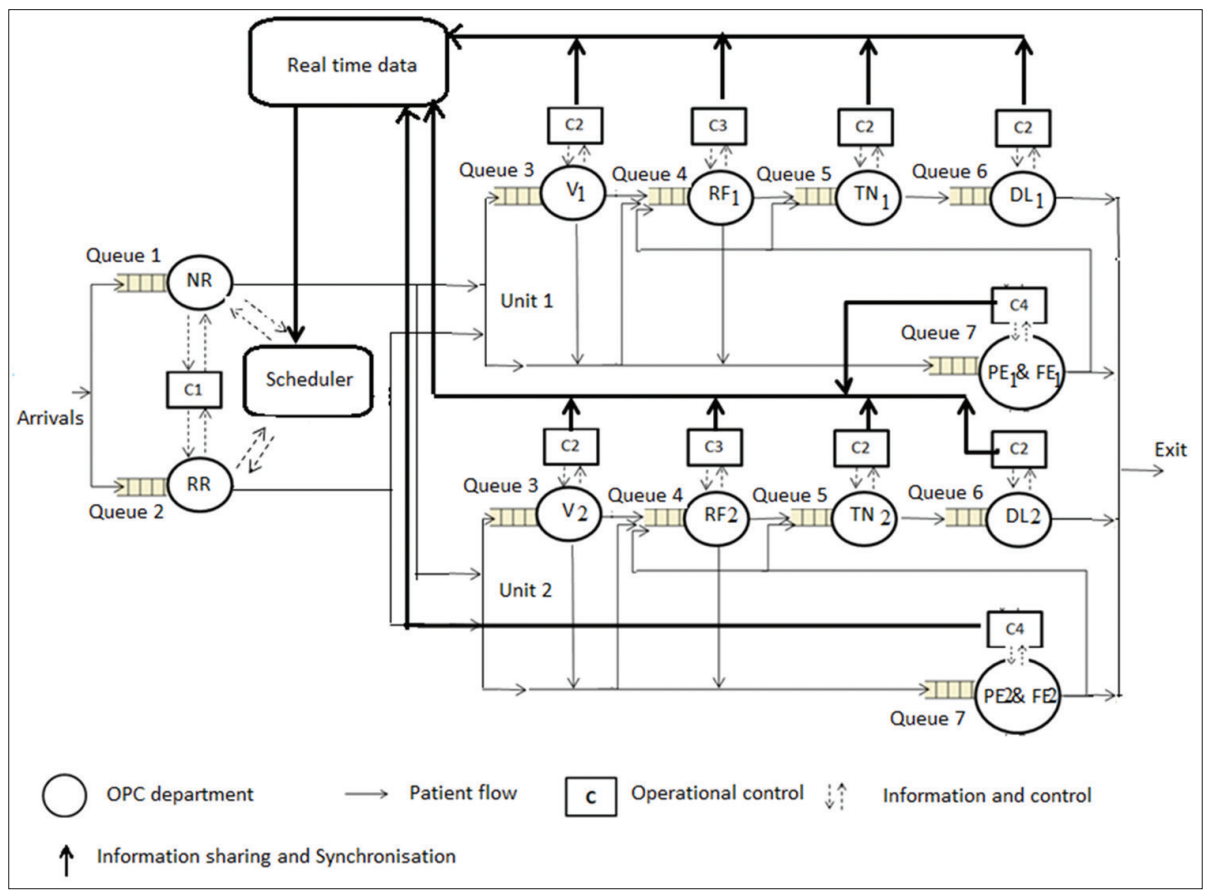

Figure 5.2: Operational control of the proposed integral patient scheduling

At the second level, we connect the scheduling and controlling tasks of all departments to the scheduler (software in IHMS) wherein upstream departments, such as NR and RRs, utilise the actual waiting time of all downstream departments. Finally, using all this system data, the scheduler finds the optimal path/schedule for the patient. The patient is scheduled at registration by considering the complete global (not departmental) pathway. Now, decisions related to sequencing and scheduling are not taken manually by the managers in the departments but are taken by the scheduler. With this scheduling model, OPCs schedule patients on arrival (in real-time) to the pathway with the least waiting time. In order to implement integral patient scheduling in AEH, we modify only Rule 3 (from scheduling patients alternatively to scheduling patients based on actual system status) keeping the rest of the rules unchanged.

It is a prerequisite that all departments constantly update their status, as this information is vital to the scheduler. In this context, "response time" is the time taken by the scheduler to obtain an optimal path. Since all patients are walk-ins, they may arrive continuously or intermittently or there may be a lull. Therefore, how often the status of departments needs to be assessed and processed forms the design aspect of the scheduler. It should ideally be continuous, but there is a trade-off between response time (cost) and optimal path. This is dynamically selected by the algorithm depending on the number of patients arrived during a particular timeframe and is explained later in this section. 


\section{Hybrid Ant Agent Patient Scheduling Algorithm}

Ant algorithms are used to find the shortest distance between two nodes or points. Ants are generated at the source node, and ants walk randomly till they reach the destination node. Each node in the network has a pheromone table and a next neighbour node set. An ant chooses its next node depending on the amount of pheromone deposition in the table ("transition rule") and stores the visited nodes list in its memory. Once the ants move to the next node, the pheromone table is updated locally. When all the ants have reached the destination, a search technique finds the best path among those generated by the ants. Only the nodes on the best path are updated by the global update rule. The pheromone value evaporates with time, making ants explorative.

The evaporation rate $(0<\alpha<1)$ plays an important role in selecting an optimal path and acts as a key point for setting up and controlling pheromones. Here, the evaporation rate equates to how often the data needs to be accessed and processed and is fixed in the ant agent algorithm. ${ }^{[37]}$ A high evaporation rate corresponds to a faster adaptation. If the pheromone evaporates too quickly, the prior knowledge collected by ants cannot be used. Hence, the ants become exploratory and the search time increases. Conversely, a low evaporation rate corresponds to slow adaptation. The ants exploit the information collected by previous ants and do not explore new paths. The dynamic environment requires an adaptive evaporation rate. Hence, we developed a self-adaptive evaporation rate for an ant agent algorithm to schedule patients in real-time. In the OPC, patients have to follow a sequence in which each department is visited. ${ }^{[23]}$ Hence, our hybrid ant agent algorithm was based on the precedence and load distribution constraints of the OPC.

In this algorithm, ants (software packets) are generated at the registration department, which virtually travel to all departments for finding an optimal path (with minimum cycle time). Generally, Tabu search is used to avoid cycle formation by the ants. We use a simple search as precedence constraints are already incorporated; hence, there is no need to handle cycle formation explicitly. The search used in this algorithm satisfies the defined objective of minimising the total travel time (patient cycle time) and consequently minimising the waiting time. We define two sets of evaporation rates: $E_{\text {low }}$ with lower values that are randomly generated from $(0.0,0.5)$ and $\mathrm{E}_{\text {high }}$ with higher values that are randomly generated from $(0.5,1.0)$. The ants, when generated, extract the time of previous/earlier ant generation and use the last status update. If either of them is more than a set threshold, then the ant will select randomly from $E_{\text {high }}$ or $E_{\text {low }}$. We explain the steps of hybrid ant agent algorithm below.

\section{Algorithmic Steps}

The algorithm runs in real-time throughout the day. 
Step 1: Define the departments as nodes and the connections between them as links. Here, we have defined 12 departments and their connections, along with the rules and constraints of the OPC.

Step 2: Generate ants at the source. In this case, two types of ants were generated (one for NR and one for RR). We chose to generate several ants for each patient due to high variability and uncertainty in patient arrivals. The possible pathways for both new and review patients in the OPC were found to be eight (four each for NR and RR), and the numbers of nodes in the OPC were less, not as many as in wireless networks. Therefore, four ants per patient were generated for finding an optimal path. The number of ants increased with the increasing number of nodes and pathways. The ants store the information about the nodes visited and the waiting time in each node.

Step 3: The ants travel from the source to the destination (registration to FE) with the pheromone table being maintained at all nodes. The pheromone table consists of a node number/ id, the number of patients in the department (in processing and queue), randomly generated service time, waiting time, precedence constraint, next feasible node and pheromone value.

Step 4: The ants use the pheromone table for selecting the next node after applying the probabilistic transition rule.

$$
\mathrm{p}_{\mathrm{ij}}=\max \frac{\tau_{\mathrm{ij}} \eta_{\mathrm{ij}}}{\sum \tau_{\mathrm{ij}} \cdot \eta_{\mathrm{ij}}}
$$

Where $p_{i j}$ is the probability of an ant in node " $i$ " selecting node " $j$ " $\forall \mathrm{j} \in \mathrm{F}(\mathrm{i}), \tau_{i j}$ is the pheromone value on the arc $i, j$ and $\eta_{i j}$ is the reciprocal of waiting time. We use the same transition rules and pheromone tables for both types of ants.

Step 5: Once the next node is selected, the current node = next node and the ants update their visited node list. The ants then update the arc that they travelled, which is called the local update of the pheromone table.

$\tau_{(i, j)}=(1-\alpha) \cdot \tau_{(i, j)}+\alpha \cdot \tau_{0}$

Where $\tau_{(\mathrm{i}, \mathrm{j})}$ is the pheromone value of the arc connecting node " $i$ " to node " $j$ " and $\alpha$ is the evaporation rate of the pheromone $(0<\alpha<1)$. This evaporation rate is selected on the basis of the frequency of ant generation. $\tau_{0}$ is the initial pheromone value at the start of the algorithm. Steps 4 and 5 are repeated until all ants reach the destination node.

Step 6: When all ants reach the destination, the time spent by them on the network is obtained with the help of their memory. 
Step 7: Once the total time spent is obtained, a search method is used to select the optimal path with the minimum time spent $\left(T_{\text {best }}\right)$ by an ant in the network. The load distribution between the units is considered before scheduling the patient to the path $\left(T_{\text {best }}\right)$. If the difference " $\Delta$ " between load U1 and load U2 is more than the threshold value, the second best path is selected. The pheromone values for nodes in the selected optimal path are updated using the global update rule.

$$
\tau_{\mathrm{ij}}=(1-\alpha) \cdot \tau_{\mathrm{ij}}+\frac{\alpha}{\mathrm{T}_{\text {best }}}
$$

Where $\left(T_{\text {best }}\right)$ is the minimum time spent by the ant in the system and the optimal path in the workflow. Ants are destroyed after providing the solution space, and new ants are generated as and when needed. Steps from 2 to 7 are repeated either till the end of the day or till all patients are scheduled.

\section{MATERIALS AND METHODS}

\section{Data Collection and Analysis}

In the first phase, data were collected by observing and interviewing hospital staff, such as ophthalmologists, paramedical staff, managers and administrative staff, to understand the workflow process. In the second phase, data were collected from the in-house software: IHMS and CMS from January 2012 to June 2012. Data of 53,802 patients was obtained that included patient demand, arrival times, in-time and out-time for units, clinical pathways and resource schedule. Other input information like waiting times, patient mix, service times and the total number of patients serviced in both units were extracted from the collected data. The probability distribution of service time and patient arrival time was determined by a data fitting tool, called Easy Fit. A Kolmogorov-Smirnov test was used to test the goodness of fit. Service times were assigned randomly between the minimum and the maximum service time from empirical data. It was observed that the patient arrival pattern had two peak loads, at $8 \mathrm{am}$ and at $10 \mathrm{am}$. Therefore, bimodal Poisson distribution ${ }^{[38,39]}$ was selected to generate patient arrival times using Equation 5.4.

$P=\left\{v_{1}, v_{2}\right\}$ and $\lambda=\left\{\lambda_{1}, \lambda_{2}, P\right\}$

Where $P$ is the sum of two Poisson distributions with mean arrivals $\lambda_{1}$ and $\lambda_{2}$ mixed with proportions: $v_{1}=0.35$ and $v_{2}=0.65$, to achieve bimodal Poisson distribution. The workflow of the model was verified by using a structured walk-through by the managers and the IT department of the OPC. 


\section{Model Development}

A discrete event simulation model of the OPC in AEH was developed by using Java. A patient was an entity, whose progress was tracked throughout the process in the OPC. The managers of the OPC verified the model. The output of the model under different input parameter settings was examined. Further, the simulation model was calibrated by increasing the number of resources (a reactive measure) to improve the accuracy in some departments during peak hours. The simulation model was run with the empirical data and performance parameters of the previous 6 months. Results from the simulation model were compared with the empirical data of the OPC for validation as shown in Table 5.1 and no statistical difference was found between the two.

\section{Experimental Design}

In the proposed model, patients were scheduled on the basis of patient arrival time and arrival pattern. Patient demand in AEH was stochastic with an average of 1800 patients/ day with $30.8 \%$ of the monthly patient demand being $1000-1600$ patients/day, $49.9 \%$ being 1600-2000 patients/day and 19.3\% being greater than 2000 patients/day. Therefore, we classified patient demand into low, medium and high. The evaporation rate in a traditional ant algorithm is fixed at a number between 0 and 1 . Since the evaporation rate affects the response time and quality of solutions, we chose to experiment with a fixed and self-adaptive evaporation rate. Therefore, this experimental design had three factors: Patient demand (three levels: Low, medium and high), patient scheduling rules (two levels: Existing and proposed/integral patient scheduling) and evaporation rate (fixed: Low and high, and self-adaptive). There were totally 18 experiments. The performance measures (waiting times, cycle times and load balance) were recorded for all the experiments. A full factorial experiment was carried out to estimate the effect of selected factors on the performance parameters.

\section{Simulation Runs}

The experiment was replicated 10 times with 180 runs to estimate the variability associated with scheduling. Simulation of a day took around 5-6 min. Different arrival times for the same

Table 5.1: Validation of the simulation model with the existing OPC in AEH

\begin{tabular}{|c|c|c|c|c|c|c|c|c|c|}
\hline \multirow[t]{3}{*}{$\begin{array}{l}\text { Patient } \\
\text { demand }\end{array}$} & \multicolumn{3}{|c|}{ Waiting time in minutes } & \multicolumn{3}{|c|}{ Cycle time in minutes } & \multicolumn{3}{|c|}{$\begin{array}{l}\text { Load distribution in number of } \\
\text { patients }\end{array}$} \\
\hline & \multicolumn{2}{|c|}{ Mean $\pm S D$} & \multirow[t]{2}{*}{$P$ value } & \multicolumn{2}{|c|}{ Mean $\pm S D$} & \multirow[t]{2}{*}{$P$ value } & \multicolumn{2}{|c|}{ Mean $\pm S D$} & \multirow[t]{2}{*}{$P$ value } \\
\hline & $\begin{array}{c}\text { Existing } \\
\text { AEH }\end{array}$ & $\begin{array}{c}\text { Simulation } \\
\text { model }\end{array}$ & & $\begin{array}{l}\text { Existing } \\
\mathrm{AEH}\end{array}$ & $\begin{array}{c}\text { Simulation } \\
\text { model }\end{array}$ & & $\begin{array}{c}\text { Existing } \\
\mathrm{AEH}\end{array}$ & $\begin{array}{c}\text { Simulation } \\
\text { model }\end{array}$ & \\
\hline Low & $48.6 \pm 12.5$ & $45.7 \pm 10.6$ & 0.5 & $98.9 \pm 14.3$ & $96.2 \pm 12.5$ & 0.4 & $3.3 \pm 1.2$ & $1.9 \pm 1.0$ & 0.4 \\
\hline Medium & $68.2 \pm 18.5$ & $66.1 \pm 19.1$ & 0.4 & $122.3 \pm 17.8$ & $119.9 \pm 20.4$ & 0.3 & $4.1 \pm 2.5$ & $1.9 \pm 1.3$ & 0.3 \\
\hline High & $82.1 \pm 25.0$ & $79 \pm 23.5$ & 0.6 & $138.9 \pm 27.1$ & $137.9 \pm 25.3$ & 0.5 & $4.3 \pm 2.8$ & $2.1 \pm 1.4$ & 0.5 \\
\hline
\end{tabular}

OPC: Outpatient clinic, AEH: Aravind Eye Hospital, SD: Standard deviation 
mean patient arrivals were generated using Random Variate (varied seed). The same randomiser input was used for simulation with two scheduling rules and evaporation rates, to ensure that the results obtained were not due to randomness. The collected mean and standard deviation of the waiting time, cycle time and load distribution are reported in the results section.

\section{Implementation of the Integral Patient Scheduler Model in AEH}

The patient scheduling algorithm was integrated into the IHMS (.NET and Visual Basic [VB] software with a MySQL database). Figure 5.3 shows the timing and data interaction during patient scheduling in the IHMS. A staff member first enters patient details in the registration form and invokes the scheduler, which requests actual status from the database. The database extracts data from all departments and sends it to the scheduler, which in turn finds the optimal path and prints it on the patient information card. The model was implemented between February 2014 and March 2014. Performance measures were collected from IHMS and CMS. ANOVA-tests were conducted on simulation and implementation results for statistical comparisons at a significance level of $0.05 \alpha$ value.

\section{RESULTS}

The mean cycle time for the existing patient scheduling and integral patient scheduling (simulation) was $120 \pm 19.7 \mathrm{~min}$ and $97.9 \pm 12.9 \mathrm{~min}$, respectively. The mean waiting time was 66.3 \pm 18.7 and $44.2 \pm 11.6 \mathrm{~min}$, respectively. The waiting time in the OPC was reduced by $33 \%$ using the integral patient scheduling. The mean and standard deviation of waiting time, cycle

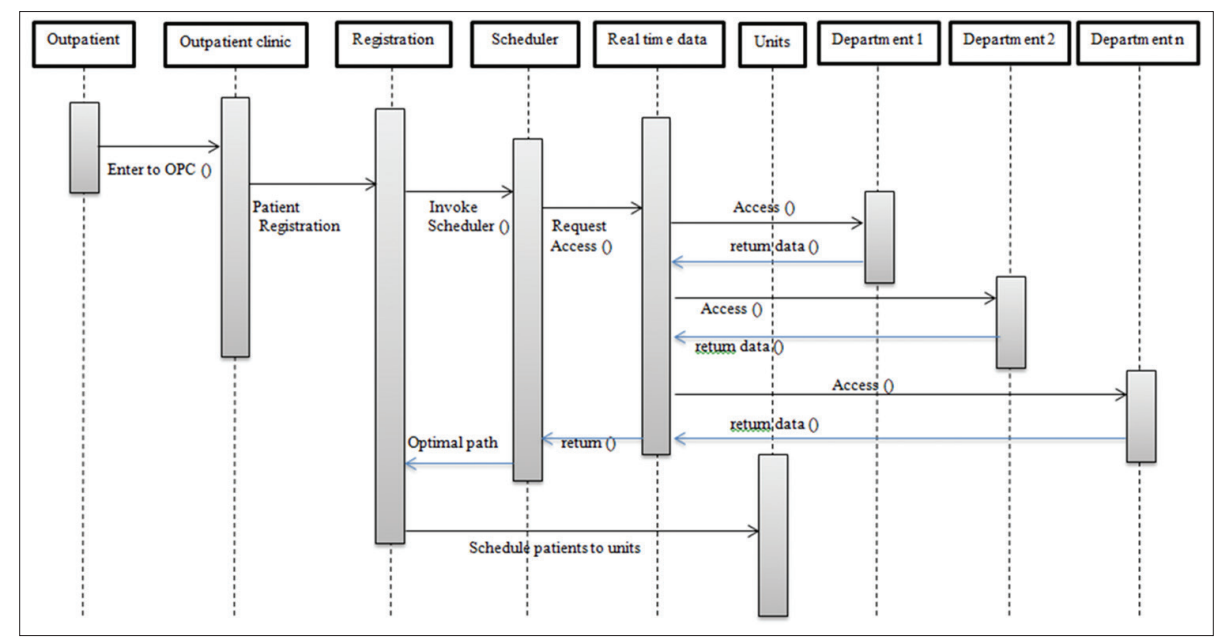

Figure 5.3: Timing and data interaction during integral patient scheduling in the integrated hospital management system of the outpatient clinics 
time and load distribution for the existing scheduling and proposed patient scheduling are compared in Table 5.2. ANOVA-tests $(P=0.05)$ show that the performance measures of integral patient scheduling are significantly better than the existing patient scheduling (Table 5.3).

The average waiting time was reduced in all departments except registration because our model is applied at registration. The waiting times were significantly reduced in PE and FE departments and refraction (Figure 5.4).

The results of existing $\mathrm{AEH}$, integral patient scheduling in the simulation model and after implementation in the OPC of AEH are compared in Table 5.3.

$13.5 \%$ of patients were found to be spending more than $2 \mathrm{~h}$ in the OPC prior to the implementation of integral patient scheduling model, which has been reduced to $8.7 \%$ after its implementation.

Table 5.2: Comparison of performance indicators for fixed evaporation rate of $0.4,0.8$ and self-adaptive

\begin{tabular}{llccc}
\hline Performance measures & Patient demand & \multicolumn{3}{c}{ Mean \pm SD } \\
\cline { 3 - 4 } & & $\alpha=0.4$ & $\alpha=0.8$ & $\alpha=$ self-adaptive \\
\hline Waiting time in minutes & Low & $39.3 \pm 11.8$ & $32.8 \pm 8.7$ & $30.9 \pm 7.7$ \\
& Medium & $57.2 \pm 15.6$ & $49.6 \pm 12.8$ & $46.9 \pm 11.2$ \\
& High & $69.4 \pm 17.1$ & $57.1 \pm 16.1$ & $54.9 \pm 15.8$ \\
Cycle time in minutes & Low & $90.1 \pm 12.5$ & $84.3 \pm 8.8$ & $81.9 \pm 9.2$ \\
& Medium & $110.1 \pm 17.1$ & $98.7 \pm 14.1$ & $98.8 \pm 14.2$ \\
Load distribution & High & $122.5 \pm 18.9$ & $117.2 \pm 16.8$ & $112.9 \pm 15.4$ \\
& Low & $17 \pm 4.2$ & $9 \pm 4.5$ & $4.9 \pm 2.4$ \\
& Medium & $21 \pm 4.8$ & $10 \pm 4.8$ & $5.2 \pm 3.2$ \\
& High & $25 \pm 4.9$ & $12 \pm 4.3$ & $6.6 \pm 3.3$ \\
\hline
\end{tabular}

SD: Standard deviation

Table 5.3: Comparison of performance measures of existing AEH, implementation of integral patient scheduler in the simulation model and after implementation in the OPC of $\mathrm{AEH}$

\begin{tabular}{|c|c|c|c|c|c|c|}
\hline \multirow[t]{2}{*}{ Performance measures } & \multirow[t]{2}{*}{ Patient demand } & \multirow{2}{*}{$\frac{\text { Existing AEH }}{\text { Mean } \pm S D}$} & \multicolumn{2}{|c|}{ Simulation model } & \multicolumn{2}{|c|}{ After Implementation } \\
\hline & & & Mean $\pm S D$ & $P$ value & Mean \pm SD & $P$ value \\
\hline \multirow[t]{3}{*}{ Waiting time in minutes } & Low & $48.6 \pm 12.5$ & $30.9 \pm 7.7$ & 0.002 & $31.9 \pm 10.2$ & 0.001 \\
\hline & Medium & $68.2 \pm 18.6$ & $46.9 \pm 11.2$ & 0.003 & $51.9 \pm 13.1$ & 0.002 \\
\hline & High & $82.1 \pm 25.0$ & $54.9 \pm 15.8$ & 0.001 & $62.4 \pm 15.9$ & 0.001 \\
\hline \multirow[t]{3}{*}{ Cycle time in minutes } & Low & $98.9 \pm 14.2$ & $81.9 \pm 9.2$ & 0.003 & $86.2 \pm 12.3$ & 0.002 \\
\hline & Medium & $122.3 \pm 17.8$ & $98.8 \pm 14.2$ & 0.003 & $109.5 \pm 14.9$ & 0.002 \\
\hline & High & $138.9 \pm 27.1$ & $112.9 \pm 15.4$ & 0.004 & $119.9 \pm 20.6$ & 0.001 \\
\hline \multirow[t]{3}{*}{ Load distribution } & Low & $3.3 \pm 1.2$ & $4.9 \pm 2.4$ & 0.04 & $5 \pm 3.3$ & 0.03 \\
\hline & Medium & $4.1 \pm 2.5$ & $5.2 \pm 3.2$ & 0.03 & $7 \pm 3.2$ & 0.04 \\
\hline & High & $4.3 \pm 2.8$ & $6.6 \pm 3.3$ & 0.04 & $9 \pm 4.1$ & 0.04 \\
\hline
\end{tabular}

OPC: Outpatient clinic, AEH: Aravind Eye Hospital, SD: Standard deviation 


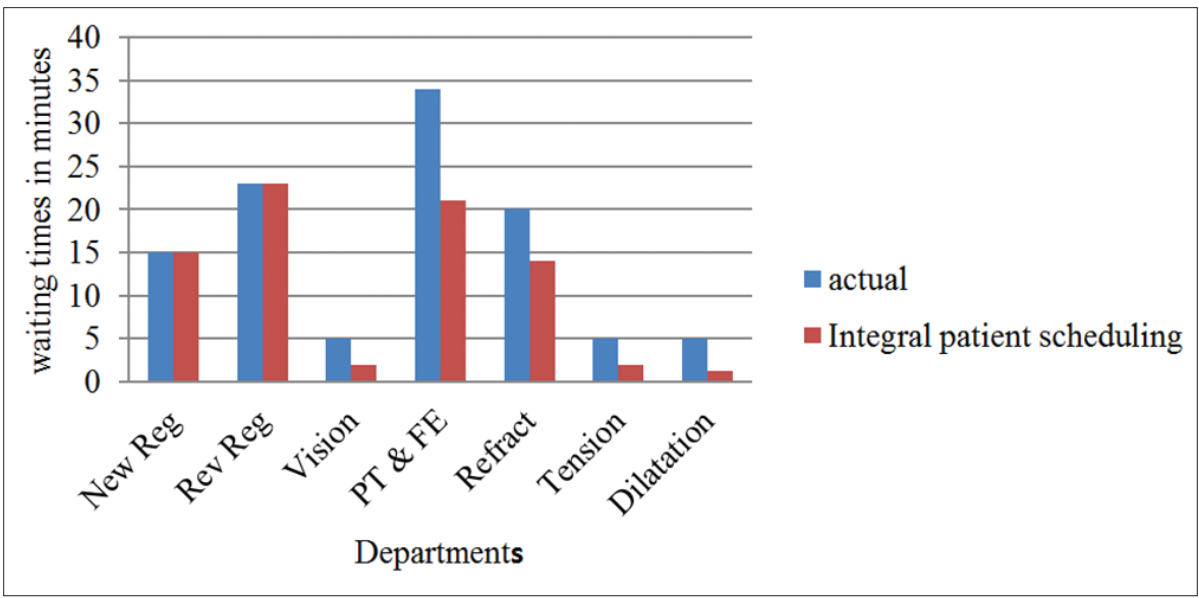

Figure 5.4: Average waiting times in all the departments in the outpatient clinic (units 1 and 2)

\section{DISCUSSION}

The OPC system in AEH, unlike other hospitals, gets only walk-in patients and schedules patients alternatively to the two units and the scheduling is local within the units. The current method of patient scheduling is department-centric; therefore, waiting times of downstream departments are unknown and not utilised by upstream departments (mainly registration). Therefore, patients wait in some departments and resources wait in some other departments.

In this study, we propose an integral patient scheduling model based on the actual status of the various departments in the OPC system with a global perspective. Our findings confirm that the proposed scheduling reduces the average patient waiting time by $33 \%$ (from 66.3 to $44.2 \mathrm{~min}$ ) in the simulation study and by $26.5 \%$ (from 66.3 to $48.7 \mathrm{~min}$ ) when implemented in the OPC of $\mathrm{AEH}$. The average cycle time reduces by $18.45 \%$ in the simulation study and by $12.3 \%$ during the implementation study. The criterion of equal load distribution (equal number of patients serviced in both units) between the two units was satisfied. Variability in patient waiting time, as well as cycle time, was reduced. Additionally, the number of patients spending more than $2 \mathrm{~h}$ was decreased from $13.5 \%$ to $8.7 \%$ (by $35.5 \%$ ). Since patients were scheduled at registration, there was no reduction in the waiting time in these departments (Figure 5.4). The waiting times in PE \& FE and refraction departments were reduced significantly. Excluding patient scheduling, other activities in the departments were controlled locally.

Patients were required to visit multiple departments and they were scheduled locally at each department without coordination among upstream and downstream departments, resulting 
in waiting times and resource under-utilisation at the same time. The scheduling model implements the global perspective with the pathway approach and schedules patients in real-time with the actual status of departments. A local optimisation might improve waiting times but not the cycle times, whereas pathway approach (global optimisation) improves both waiting times and cycle times. ${ }^{[19]}$

Patient scheduling, when performed locally (local to departments) and ahead of time (through appointment systems), does not address operational problems like waiting times. The difference between planning and actual scenario, limited knowledge (local) and lack of coordination among departments results in waiting time. The number of patient arrivals is high at a particular time of the day and low at other times. Even with high and low demand, there is variability in the arrivals. The evaporation rate relates to how often data is accessed from the departments. It is advantageous if ants are exploratory, but accessing and processing real-time data costs time and should be performed optimally. When the arrivals are faster, the generation of ants is frequent and the information collected (pheromone value) can be used by later ants. However, when there is a delay between generation of ants, the system status changes with time and needs to be re-assessed.

The evaporation rate in the ant algorithm influences the exploratory nature of ants. The results of waiting time with an evaporation rate of 0.8 (data was accessed and processed on arrival of each patient) and self-adaptive, did not show a significant difference (see Table 5.3). It indicates that when the system/network has a high variability, the ants need to be exploratory to find optimal paths. The number of nodes (departments) is small and few ants are generated at a time, because of deterministic pathways. The life cycle of ants is shorter; hence, the high evaporation rate does not affect the response time. The selection of evaporation rate depends on patient volume, arrival pattern, pathway and complexity of the OPC. The self-adaptive evaporation rate optimises the frequency of real-time data access that would be beneficial in complex hospitals with more departments.

The response time can increase exponentially with the increase in the number of pathways and departments (50 or 100) in a hospital. Therefore, to extend this model to other types of clinics or hospitals, the response time should be studied in detail. The pathways in specialised hospitals or OPCs are more deterministic and less variable than one would expect in general hospitals.

The load distribution between the two units was maintained throughout the day, with very few differences. The evaporation rate also affects the load distribution of the units, where a lower value creates more difference. As $\alpha$ was self-adaptive in this study and load distribution was one of the necessary criterions to be satisfied, we explicitly included this in the algorithm. 
Based on the results of the ANOVA test $(P=0.05)$ that was conducted on waiting time and cycle time, we reject the null hypothesis. Overall optimisation using integral patient scheduling improves waiting time and cycle time in the OPC.

The literature shows that patient scheduling often dealt with appointment scheduling and only a few researchers have addressed the issues and solutions of walk-in patient ${ }^{[14]}$ by reserving a few time-slots for them. Such scheduling failed to incorporate the variability in short term (actual system status) and complete pathways. In this study, the integral patient scheduling model incorporates global optimisation along with actual departmental status. It schedules patients in such a way that the total time spent by them in the OPC is minimised. We have also incorporated a self-adaptive evaporation rate that makes it a self-analysing and self-correcting network, where the slightest change in the OPC environment could be managed effectively. This model also helps OPCs at the operational level, which means that whenever a bottleneck arises (due to the breakdown of equipment, staff problems, etc.) it schedules the patients to a congestion-free pathway. The model fits in online operational planning and control level of the framework. The model analyses all the possible alternatives quickly under time pressure and strengthens the operational decisions in patient scheduling.

Scheduling patients in real-time not only depends on the algorithm but also on the IT structure and knowledge management system of the OPC. In this study, the hospital staff in each department updated the patient and process data. As a future work, it is suggested to utilise radio frequency identification tags for patients to update the status and explore the learning mechanism for selecting an effective evaporation rate. We also intend to explore the integral patient scheduling model in complex hospitals with stochastic pathways.

\section{CONCLUSION}

This study shows how integral patient scheduling improves waiting times and cycle times in OPCs. This study integrates two aspects of scheduling: Actual status driven and global perspective. The integral patient scheduling overcomes the problem of unregulated waiting times and prolonged cycle times by global optimisation that is implemented through the pathway approach.

Patient scheduling in open loop systems like OPC systems is extremely important in managing waiting time, which is a quality care measure. Our experiments establish the importance of real-time information and its sharing, coordination among departments and global optimisation in the performance of an OPC. This scheduling model has been implemented to an eye care OPC with deterministic pathways and can probably be extended to general hospitals by considering their workflow complexities. 


\section{REFERENCES}

1. Oche M, Adamu H. Determinants of patient waiting time in the general outpatient department of a tertiary health institution in north Western Nigeria. Ann Med Health Sci Res 2013;3(4):588-92.

2. Huang XM. Patient attitude towards waiting in an outpatient clinic and its applications. Health Serv Manag Res Off J Assoc Univ Programs Health Adm HSMC AUPHA 1994;7(1):2-8.

3. Mageshwari G, Kanaga EG. Literature review on patient scheduling techniques. Int J Comput Sci Eng IJCSE 2012;4(3):397-401.

4. Bailey NT. A study of queues and appointment systems in Hospital out-patient departments, with special reference to waiting times. J R Stat Soc Ser B Methodol 1952;14(2):185-99.

5. Harper PR, Gamlin HM. Reduced outpatient waiting times with improved appointment scheduling: A simulation modelling approach. OR Spectr 2003;25(2):207-22.

6. Cayirli T, Veral E. Outpatient scheduling in health care: A review of literature. Prod Oper Manag 2003;12(4):519-49.

7. Byron HM. The ophthalmologist's office: Planning and practice. Effective patient scheduling. Int Ophthalmol Clin 1975;15(2):85-93.

8. Berg BP, Denton BT, Erdogan SA. Thomas rohleder and todd huschka, optimal booking and scheduling in outpatient procedure centers. Comput Oper Res 2014;50:24-37.

9. Friedman JP. Internet patient scheduling in real-life practice. J Med Pract Manag 2004;20(1):13-5.

10. Legrain A, Fortin MA, Lahrichi N, Rousseau LM. Online stochastic optimization of radiotherapy patient scheduling. Health Care Manag Sci 2015;18(2):110-23.

11. Holleman DR Jr, Bowling RL, Gathy C. Predicting daily visits to a walk-in clinic and emergency department using calendar and weather data. J Gen Intern Med 1996;11(4):237-9.

12. Cayirli T, Gunes ED. Outpatient appointment scheduling in presence of seasonal walk-ins. J Oper Res Soc 2014;65(4):512-31.

13. Peng Y, Qu X, Shi J. A hybrid simulation and genetic algorithm approach to determine the optimal scheduling templates for open access clinics admitting walk-in patients. Comput Ind Eng 2014;72(1):282-96.

14. Su S, Shih CL. Managing a mixed-registration-type appointment system in outpatient clinics. Int J Med Inf 2003;70(1):31-40.

15. Kortbeek N, Zonderland ME, Boucherie RJ, Litvak N, Hans EW. Designing cyclic appointment schedules for outpatient clinics with scheduled and unscheduled patient arrivals. Perform Eval 2014;80:5-26.

16. Baril C, Gascon V, Cartier S. Design and analysis of an outpatient orthopaedic clinic performance with discrete event simulation and design of experiments. Comput Ind Eng 2014;78:285-98.

17. Yang X, Han R, Guo Y, Bradley J, Cox B, Dickinson R, et al. Modelling and performance analysis of clinical pathways using the stochastic process algebra PEPA. BMC Bioinformatics 2012;13(14):1-17.

18. Schrijvers G, van Hoorn A, Huiskes N. The care pathway: Concepts and theories: An introduction. Int J Integr Care 2012;12:e192.

19. Ludwig M, van Merode F, Groot W. Principal agent relationships and the efficiency of hospitals. Eur J Health Econ 2010;11(3):291-304.

20. Kanaga EG, Valarmathi ML, Rose JD. Coordinated multi-agents based patient scheduling using genetic algorithm. ACEEE Int J Commun 2010;3(2):19-20.

21. Du G, Jiang Z, Yao Y, Diao X. Clinical pathways scheduling using hybrid genetic algorithm. J Med Syst 2013;37(3):9945.

22. Dorigo M, Gambardella LM. Ant colony system: A cooperative learning approach to the traveling salesman problem. IEEE Trans Evol Comput 1997;1(1):53-66.

23. Gambardella LM, Dorigo M. An ant colony system hybridized with a new local search for the sequential ordering problem. INFORMS J Comput 2000;12(3):237-55.

24. Liu Z, Kwiatkowska MZ. Costas Constantinou, A biologically inspired QoS routing algorithm for mobile ad hoc networks. In: $19^{\text {th }}$ International Conference on Advanced Information Networking and Applications (AINA) IEEE; 2005.

25. Ren $\mathrm{H}$, Meng MQ. Biologically inspired approaches for wireless sensor networks. In: IEEE International 


\section{Chapter 5}

Conference on Mechatronics and Automation, IEEE; 2006.

26. Rosati L, Berioli M, Reali G. On ant routing algorithms in ad hoc networks with critical connectivity. Elsevier Ad Hoc Netw 2008;6(6):827-59.

27. Sum J, Shen H, Leung CS, Young G. Analysis on a mobile agent-based algorithm for network routing and management. IEEE Trans Parallel Distrib Syst 2003;14(3):193-202.

28. Sim KM, Sun WH. Ant colony optimization for routing and load-balancing: Survey and new directions. IEEE Trans Syst Man Cybern A Syst Hum 2003;33(5):560-72.

29. Bean N, Costa A. An analytic modelling approach for network routing algorithms that use "ant-like" mobile agents. Elsevier Comput Netw 2005;49(2):243-68.

30. Chaudhary B, Modi AG, Reddy K. Right to sight: A management case study on Aravind Eye Hospitals. Zenith Int J Multidiscip Res 2012;2(1):447-57.

31. Rangan VK, Thulasiraj RD. Making sight affordable (innovations case narrative: The Aravind eye care system). Innov Technol Governance Global Fall 2007;2(4):35-49.

32. Andersen MM, Poulfelt F. Beyond Strategy: The Impact of Next Generation Companies. New York: Routledge; 2014.

33. Natchiar G, Thulasiraj RD, Sundaram RM. Cataract surgery at Aravind Eye Hospitals: 1988-2008. Community Eye Health 2008;21(67):40-2.

34. Brilliant L, Brilliant G. Aravind: Partner and social science innovator (innovations case discussion: Aravind eye care system). Innov Technol Governance Global 2007;2(4):50-2.

35. Mehta PK, Shenoy S. Infinite Vision: How Aravind Became the Greatest Business Case for Compassion. San Franciso: Berrett-Koehler Inc.; 2011. p. 336.

36. Aravind Eye Care System: Activity Report. Madurai; 2014-2015. p. 76.

37. Mavrovouniotis M, Yang S. Ant colony optimization with immigrants schemes for the dynamic travelling salesman problem with traffic factors. Appl Soft Comput 2013;13(10):4023-37.

38. Li J, Zha H. Two-way poisson mixture models for simultaneous document classification and word clustering. Comput Stat Data Anal 2006;50(1):63-80.

39. Karlis D, Xekalaki E. Mixed poisson distributions. Int Stat Rev 2005;73:35-58. 


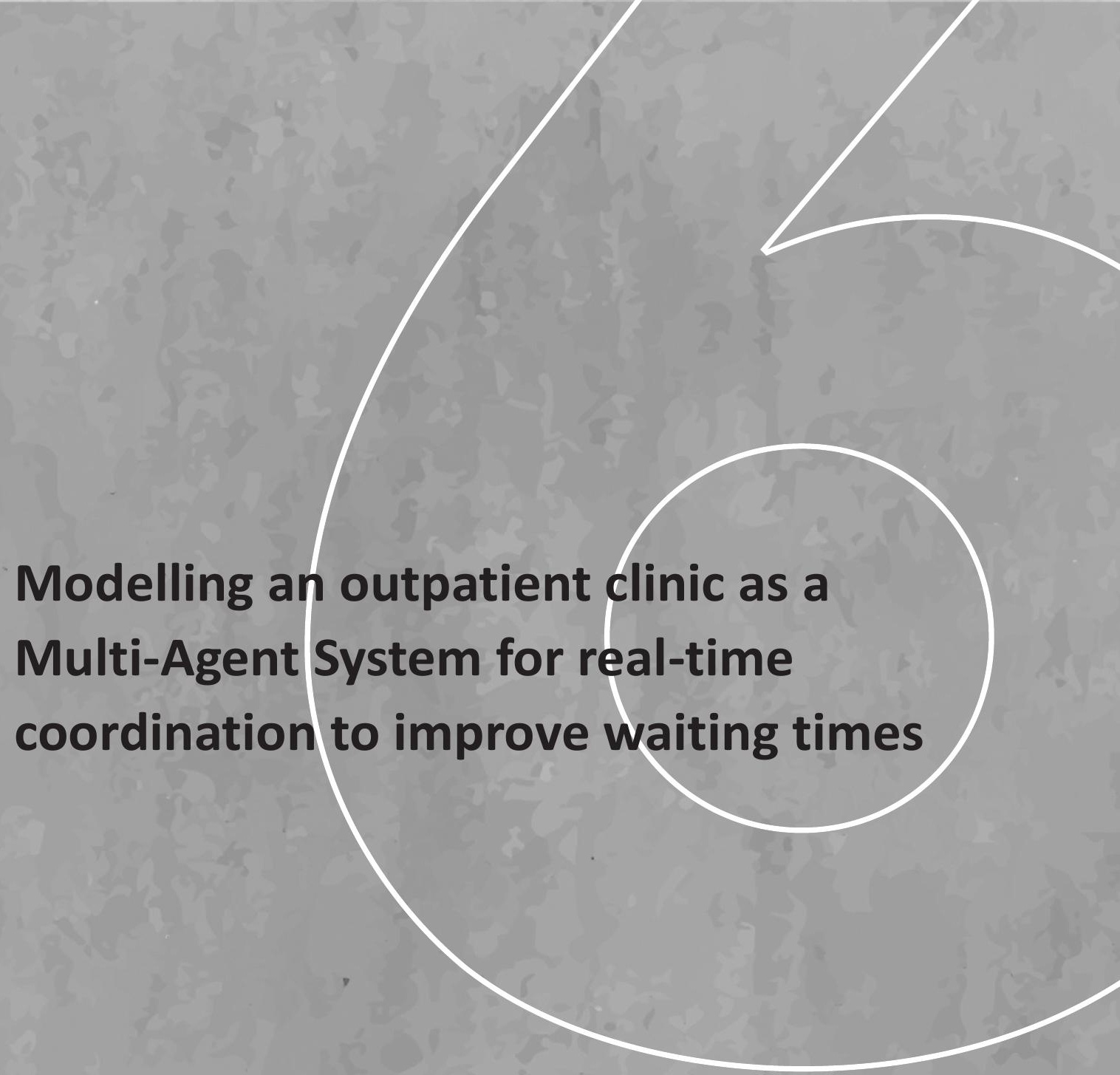

Submitted for publication:

Munavalli JR, Rao SV, Srinivasan A, van Merode GG. 



\section{INTRODUCTION}

Coordination between resources and departments has become extremely important in operations management of outpatient clinics (OPCs), as demand for outpatient care has been increased due to various reasons like an ageing population, complex medical conditions, limited resources, etc. A high demand and stagnant capacity cause higher waiting times and negatively affect patient satisfaction. ${ }^{[1,2]}$ An OPC is a system with various departments, resources, patients, logistics, information systems and most importantly, human interactions along with different groups of people like the staff, patients and the management. Each group has its own goals and limitations, which sometimes conflict. A patient's goal is to get quality service in time, and a manager's goal is to optimise quality, cost, flow and efficiency. ${ }^{[3-5]} \mathrm{An} \mathrm{OPC}$ is a loosely coupled system where all departments are often managed independently (locally), clinically as well as administratively. ${ }^{[6,7]}$ In order to achieve departmental efficiency, managers take decisions with limited knowledge about the processes and patients that might be functional elsewhere in the OPC. They might not communicate or share process information and might not coordinate as a system. Due to lack of coordination, some departments are overcrowded while some are idle. Patients wait in long queues of the overcrowded departments when they could be receiving a part of their treatment in less crowded ones. Some departments work faster than other departments. The faster departments push patients to the slower ones, where patients wait for a long time. Although local control sometimes improves departmental efficiency, it might be sub-optimal from the OPC perspective. It might reduce waiting time in certain departments, but not necessarily the cycle time (which includes registration time, service time and waiting time in all departments). ${ }^{[8,9]}$

OPCs being open systems are influenced by their environment and experience variability and uncertainties due to varying service times and random patient arrivals. OPCs schedule resources ahead of time (weekly, monthly or bi-monthly), before the arrival of patients and resources are determined at an aggregate level[10-12] because of which planning and scheduling fail to incorporate the variability and uncertainty. The management hierarchy limits the decision making by frontline employees who actually deal with variability and uncertainty. ${ }^{[13-16]}$ The resource scheduling, based on average demand and average service times, does not fully reflect reality. ${ }^{[17]}$ The demand and supply mismatch results in prolonged waiting time and under-utilisation. OPCs often view these frustrating delays as a capacity problem rather than as poor management. ${ }^{[18,19]}$ Additionally, workflow complexity increases as OPCs share scarce resources among different departments for optimising cost, due to which patients wait for resources and resources wait for patients at different locations in the OPC. ${ }^{[8]}$

OPCs schedule patients according to the availability of resources (already planned and scheduled) mostly by appointment scheduling systems. ${ }^{[20-24]}$ Time spent by patients in each department significantly varies on the basis of the complexity of their medical condition. Patients 
have to visit multiple departments for various tests, diagnosis or treatment. ${ }^{[25,26]}$ Patients are scheduled at registration or at other departments. Resource scheduling and patient scheduling are often isolated. ${ }^{[27-29]}$ OPCs lack coordination in global perspective (entire care chain/ process of the patient) and do not act on the actual status of the OPC system (pull system). Therefore, there is a need to design an OPC system that coordinates resources globally and in real-time. We propose a real-time coordination mechanism for the scheduling of resources and patients by incorporating their actual status.

OR-based methods are often too limited in representing real-world problems and lack adaptability and reactivity to dynamic changes in the workflow/external environment. In literature, it is observed that multi-agent systems (MASs) are a promising approach to overcome such limits. ${ }^{[30,31]}$ MASs have been used in patient scheduling, where patients as agents compete for treatment appointments. ${ }^{[32-34]}$ In order to achieve this purpose, the resource agents (RAs) auction off time-slots corresponding to their capacity. If the resource is free, its next time-slot is assigned to the patient agent with the highest bid. Defined utilities improve the patient's health by providing earlier appointments. ${ }^{[32,35-38]}$ Agents also negotiate with each other over scarce resources. Variable pathways in an OPC can be assessed and handled efficiently. Based on the distributed environment and information about the system, efficiency is increased by rescheduling the pathways. ${ }^{[3,34]}$ Štiglic and Kokol ${ }^{[39]}$ have scheduled patients and nurses on the basis of adaptive scheduling in the long term. The nurse scheduling is performed on the basis of workload and patient predictions on a weekly basis by using a MAS. Coordination can be achieved not only within the hospital but also between hospitals. Resource sharing and interaction between them provide better and timely care to patients. The interactions between the people in OPCs can be represented by a MAS where agents interact and cooperate to collectively solve problems. ${ }^{[40,41]}$ A MAS works efficiently and effectively when the environment is highly complex, dynamic and distributed. ${ }^{[42,43]}$ If control and responsibilities are shared sufficiently among different agents, the system can tolerate failures by one or more agents, and it can be self-correcting.

The OPC in Aravind Eye Hospital (AEH) was a good test case to experiment with a real-time coordination mechanism as it fits in the assumed working conditions. All patient arrivals are random (no appointment systems are used) and independent. This makes patient demand highly variable and uncertain. Additionally, resources are scheduled much ahead of time based on average demand. The OPC has no control on input, as the OPC provides same-day care for all patients. The OPC consists of different types of people having different goals and has a hierarchical decision-making approach. Different people play games to sub-optimally achieve their individual goals with respect to OPC-wide performance. A game is a formal description of a strategic situation. In this context, strategic means that people make choices from all possible actions and take decisions to improve their situation. In this study, the OPC is viewed as collections of more than one type of agents, such as patient, doctor and manager, 
with independent goals. These agents interact and negotiate towards achieving OPC-wide (global) goals. Next, we present resource and patient scheduling in the OPC, and then we state the hypothesis of this study.

\section{Scheduling and Coordination in the OPC in AEH}

AEH is a renowned eye care hospital in Madurai, South India, which provides patient centred care. ${ }^{[44-46]} \mathrm{AEH}$ has performed 401,529 surgeries and treated 2,396,864 outpatients during 2014-15. ${ }^{[47]}$ The hospital runs with strict quality norms, process standardisation, cost control and above all, high patient volume. AEH resembles an assembly line system (a line of workers and equipment along which a product being assembled passes consecutively from one operation to the other until completed). ${ }^{[46,48-50]}$ However, some aspects of the OPC, such as local control of operations in departments and uneven patient demand, differ from assembly line systems. It has well trained and dedicated resources (ophthalmologists and paramedical staff). The OPC is open from 7 am to $6 \mathrm{pm}$ and follows the rule: Zero at 10, which means that the OPC provides care by 10 am to patients who arrive before $9 \mathrm{am}$. For this purpose, OPC transfers resources with the same skills from various speciality clinics to the OPC, based on their availability. However, this is done manually and only as a reaction to situations where the patient load is high and the queues are building up. The OPC has its in-house software Integrated Hospital Management System (IHMS) and Clinical Management System (CMS) that record process and patient information.

In this study, we consider an OPC with two identical units. Departments, such as new registration (NR) and review registration (RR) are common to both units, and each unit has five departments, namely, vision (V), refraction (RF), tension (TN), dilatation (DL) and preliminary and final examination (PE and FE). For clarity, only unit 1 is shown in Figure 6.1. All the queues (1-7) are on the basis of first-come first-served. The patient flow arrows show the possible pathways for new and review patients. Now, we describe the current method of resource and patient scheduling in the OPC and then present its decision-making control.

The OPC in Figure 6.1 follows the rules in Box 6.1. Managers are the local controllers (C) who plan, schedule and control the activities in the departments. Different managers are responsible for scheduling the resources ( $r$ ) like ophthalmologists and paramedical staff. The OPC schedules the ophthalmologists on the basis of their availability after academic (teaching and research) activities and surgery schedules, and this scheduling is performed once in a month, ahead of time. Identical departments in both units have the same number of resources, which remains fixed throughout the day and month (Rule 3 and 5). During peak hours, managers (controllers) apply rules 4 and 9 to the situation. Based on their experience, they increase resources in their departments or shorten lunch breaks of staff to control waiting times $w(t)$. When upstream departments work faster, the patients flood the downstream departments, 


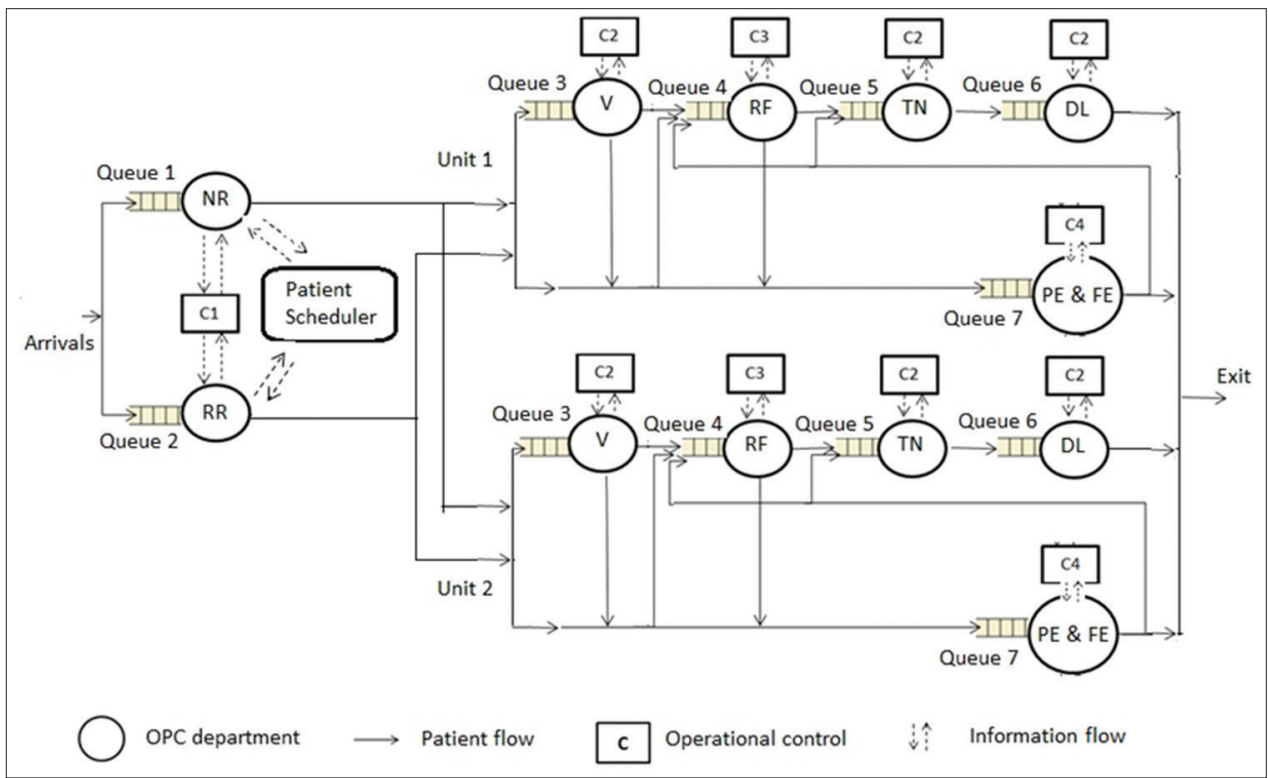

Figure 6.1: Functional structure and operational control in outpatient clinic system of Aravind Eye Hospital

Box 6.1: Rules in the OPC in AEH

Rule 1: All patients are provided with the service/care on the same day of arrival.

Rule 2: All the queues are on the basis of FCFS.

Rule 3: Resource planning and scheduling time window is 1 month.

Rule 4: Resources are scheduled by managers locally (department-centric).

Rule 5: Number of resources in unit 1 = number of resources in unit 2.

Rule 6: Patients are scheduled to units 1 and 2 alternatively.

Rule 7: The load distribution is equal to both units.

Rule 8: Patients should complete RF and PE before TN.

Rule 9: If (Queue $n>$ threshold workload) $\rightarrow$ (Resources " $r$ " is added to $n^{\text {th }}$ department $D_{n}$ such that $r \leq R_{T}$, the total number of resources) OR shorten the lunch break time of already working resources.

Rule 10: Control of operations is local (department-centric).

OPC: Outpatient clinic, FCFS: First come first serve, RF: Refraction, PE: Preliminary examination, OPC: Outpatient clinics, AEH: Aravind Eye Hospital, TN: Tension

which are unready to handle the increased workload. Similarly, when upstream departments work slower, the downstream departments wait for patients. The lack of coordination among departments increases the unregulated waiting time in some departments and under-utilisation in the same or other departments. The time taken by the manager to react to the change and take corrective measures is called the reaction time $r(t)$ (an exogenous variable) that affects the waiting time. The reaction time varies as the resources to be transferred may be busy elsewhere. Therefore, reaction time depends on the availability of resources when needed and the kind of managerial measures taken. 
The patient workflow in the OPC starts with registration and finishes with a FE. The scheduler in the IHMS schedules patients to the units during registration. OPC schedules patients alternately to units 1 and 2, so that both units have equal load distribution (rules 6 and 7). The patient moves through various departments in either of the two pathways: NR-V-PE-RFTN-DL-FE for new patients and RR-PE-RF-TN-DL-FE for review patients. The order of RF and $\mathrm{PE}$ can be interchanged. Around $5 \%$ of the patients exit after PE.

There are different groups of resources in the OPC, as shown in Figure 6.2. Mid-level ophthalmic personnel (MLOP), medical records department (MRD) staff and ophthalmologists are the frontline employees at the operational level. Each member of the MLOP group has similar roles and this group is shared among three departments (vision, tension and dilatation). An MLOP manager controls this group through an MLOP head. Another MLOP group meant exclusively for refraction is controlled by MLOP manager through the refraction MLOP head. The senior and junior ophthalmologists are shared between PE and FE and are controlled by the ophthal manager. The MRD staff members (NR and RR) are scheduled by the MRD manager. All managers in the OPC report to the patient care manager. As the decision control in the OPC is hierarchical, managers take the decisions regarding planning and scheduling; whereas, the frontline employees face the challenges at the operational level. The patient care manager takes reactive measures when problems arise. The frontline employees follow the instructions and do not take scheduling decisions. The limitations on decision control and lack of coordination resulted in inefficiency in operations.

Though the OPC in AEH is efficient, it still faces operational problems like long waiting times, cycle times and under-utilisation. The OPC, like other hospitals (Chapter 2, literature review), is an open loop system with unstable demand and lacks coordination among resources to synchronise departments in the OPC. In order to improve operations, there is a need for coordination of resources so that scheduling can be in real-time. Accordingly, resources should adapt to the system changes due to variability and uncertainty in demand. ${ }^{[51]}$ Therefore, we develop a real-time coordination mechanism that schedules resources globally, based on actual demand. In this approach, we integrate global resource scheduling with real-time coordination.

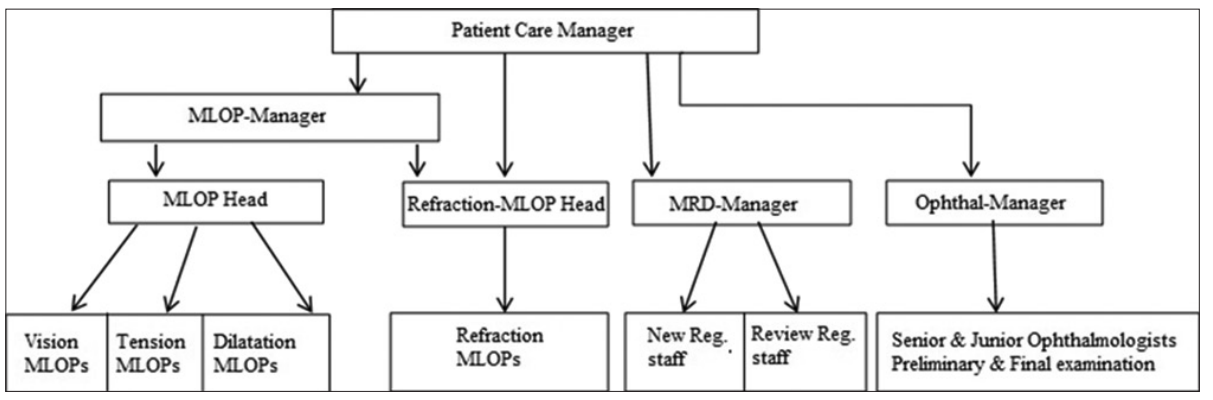

Figure 6.2: Decision control in the outpatient clinic 
We state the hypothesis of this study:

"An OPC system with disparate subsystems can reduce cycle/waiting time by global optimisation, by coordinating resources and patients in real time."

This paper shall continue as follows: In Section 6.2, we present a real-time coordination mechanism for scheduling patients and resources and developing a MAS model for the OPC system. We define agents and develop algorithms to implement interactions and negotiations. Section 6.3 describes the data collection, model development, experimental design, simulation and implementation study. In Section 6.4, we present the results from different scenarios created from real case studies. We discuss our findings in Section 6.5 and conclude in Section 6.6.

\section{REAL-TIME SCHEDULING MODEL}

In this section, we describe scheduling of resources and patients along with coordination mechanism, all in real-time. The model uses four control levels, as shown in Figure 6.3. At the first level, a pool keeps a record of all resources. At the second level, real-time data is collected

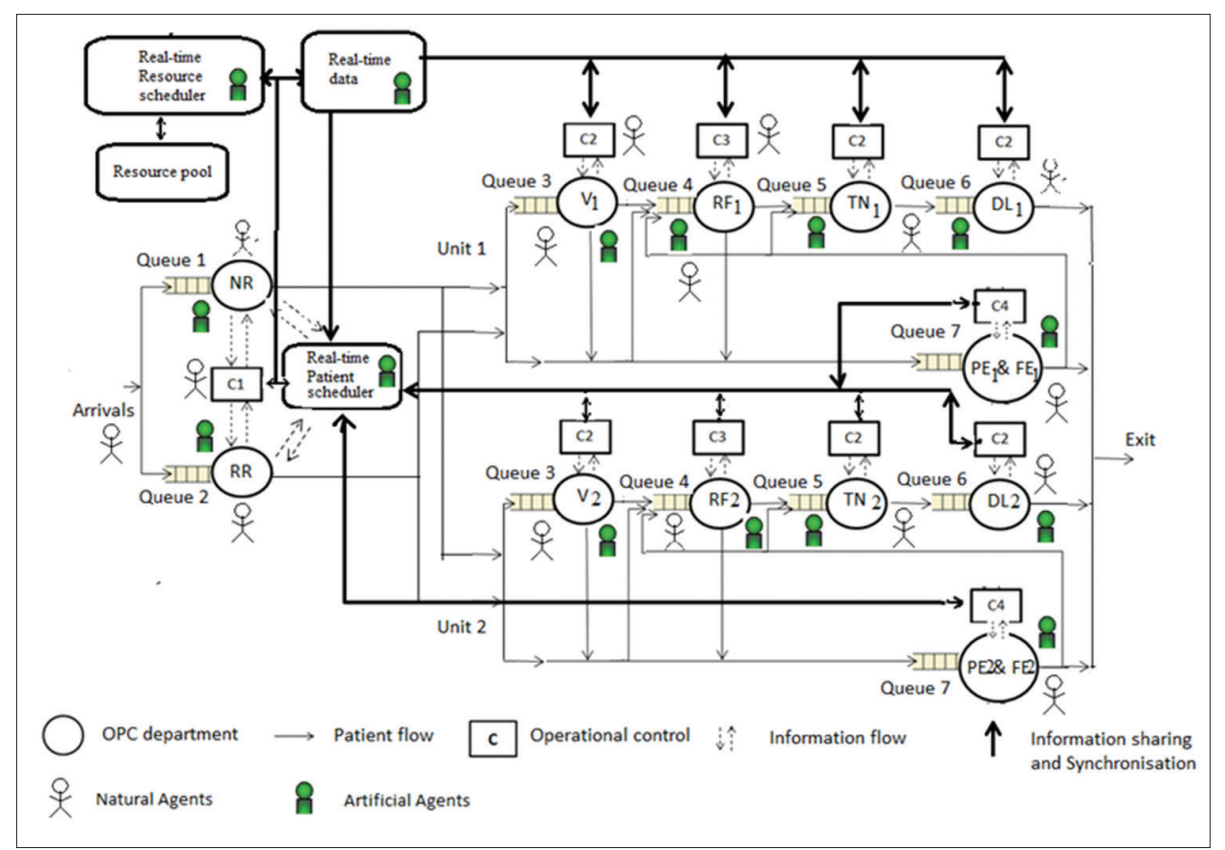

Figure 6.3: Control and functional structure in real-time resource and patient scheduling in the outpatient clinic system 
from all departments of the two units. At the third level, patients are scheduled to pathways through an integrated patient scheduler on the basis of the departmental system status. Finally, resources are scheduled. We develop a coordination mechanism where resources bid to increase their utilisation. We modify some existing rules of the OPC (Box 6.2) for implementing the proposed model. Rules 1, 2, 7 and 8 remain the same, and rules 5 and 9 are eliminated.

The participants in the OPC are mapped to agent roles in the MAS. Patients, resources in each department and managers (C1, C2 and $C 3)$ in Figure 6.3 are mapped as natural agents and additional artificial agents are created to implement the coordination mechanism. Departments, patient scheduler, route agents (RoAs) and resource scheduler were defined to facilitate the necessary information and control operations and were mapped as artificial agents. First, we describe the roles of all agents in the MAS and then present the coordination mechanism.

\section{Types of Agents}

- Patient agent (PA): These agents need care in the OPC. The database is maintained to track their movement in the departments according to clinical pathways

- Resource agent (RA): Ophthalmologists, MRD staff and paramedical staff, such as MLOP staff, are the RAs that perform the activities/tasks. The goal of these agents is to maximise their utilisation

- Manager agent (MA): Monitors the RAs in their departments

- MLOP head agent: Follows instructions from MLOP-MA

- Department agent (DA): Keeps track of all patients and resources in the department

- RoA: This agent assesses the waiting time in each department

- Patient scheduler agent: This agent creates RoAs who traverse the departments to find the optimal path for PAs

- Resource scheduler agent (RSA): This agent represents the patient care manager in the OPC. We have added the tasks of monitoring the waiting time in all departments. It also monitors the resource pool and DAs and identifies the resource requirements in each department. This agent calls for bids from resources and schedules resources to reduce waiting time in the departments. This agent gives instructions to the MA.

Box 6.2: Rules proposed by real-time scheduling model to OPC

Rule 3: Resource scheduling time window is every day.

Rule 4: Real-time scheduler schedules resources. Managers follow its instructions and monitor results.

Rule 6: Patients are scheduled on the basis of waiting time in both units.

Rule 7: Load distribution in both units is equal.

Rule 10: Control of operations with respect to resources and patient scheduling is global (OPC-centric).

OPC: Outpatient clinic 
The agents have attributes like name, address and identification number as shown in the class diagram (Figure 6.4). An agent's class consists of agent's name, services and goals. The PA has database entries regarding the departments visited, in-time and out-time. Whenever the patient enters the OPC, the class application uses an interface agent and connects all other agents with the class application. The department, patient, resource and resource pool information are shared with the RSA. Now, we present algorithms for patient scheduling and resource scheduling.

\section{Algorithm 1: Real-time Patient Scheduling Algorithm}

A record for each PA is created when a patient enters the OPC. The database stores patient ID, age, in-time, departments visited and out-time. At registration, the PA is scheduled to one of the pathways after the following actions are performed. This algorithm runs throughout the day.

- The scheduler agent creates RoAs

- RoAs gather waiting time information for all departments

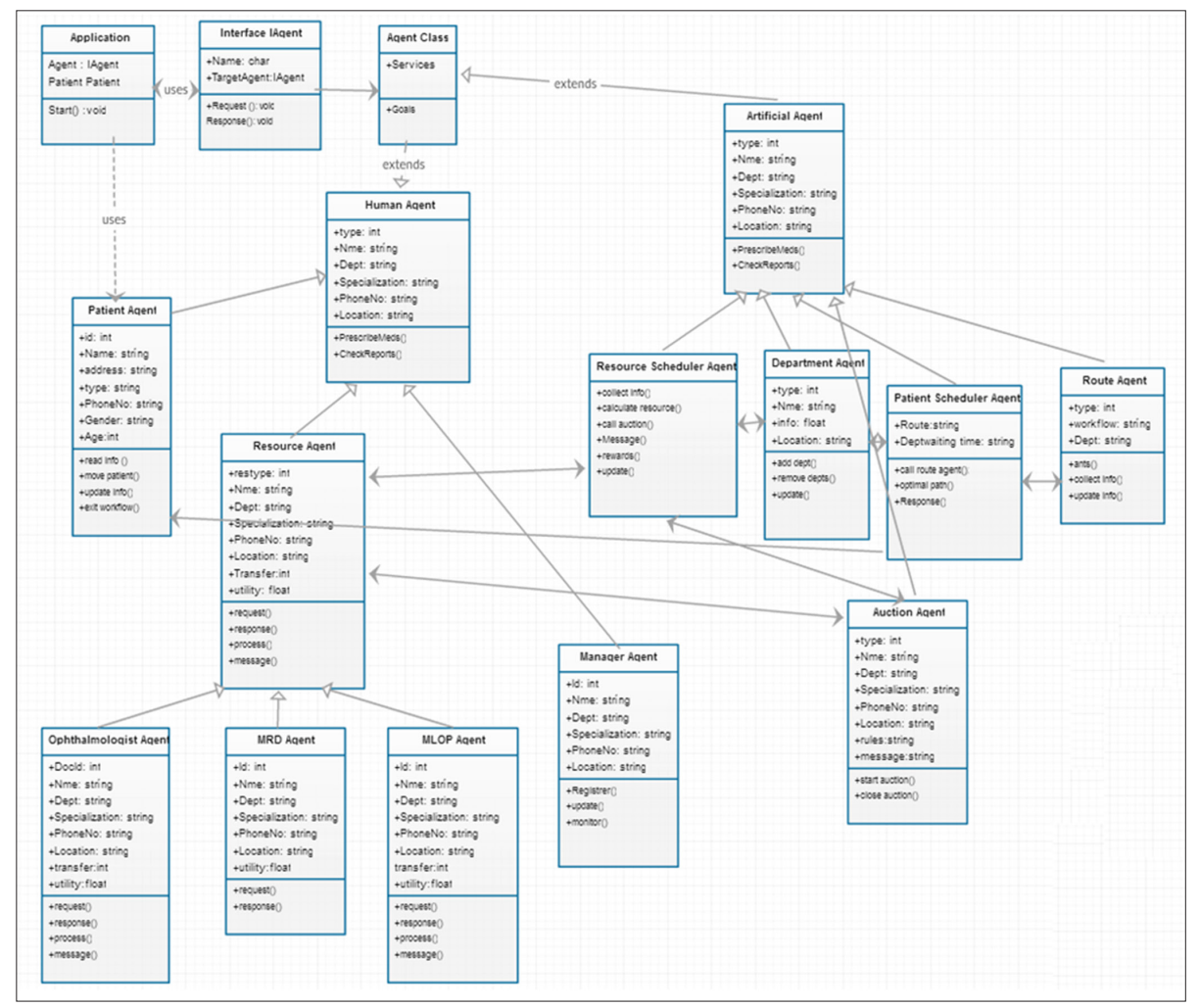

Figure 6.4: Class diagram for agents in the outpatient multi-agent system 
- The scheduler agent uses the collected information from RoAs and then chooses the pathway with the minimum wait time and schedules the PA to that pathway. The optimal path is identified by using an ant algorithm. ${ }^{[52,53]}$

\section{Algorithm 2: Real-time Resource Scheduling Algorithm}

First, we define Takt time as the time between units of output to be synchronised with the customer demand. In the OPC context, it translates to the average time at which a patient moves out of the OPC.

Takt time $=\frac{\text { Effective available time in a day }}{\text { Number of patients serviced in a day }}$

Takt time is one-way to synchronise demand and supply, commonly used in production industries. For example, consider a department with service time of $5 \mathrm{~min}$ that has to provide care for 30 patients $/ \mathrm{h}$. In this case, Takt time $=60 \mathrm{~min} / 30$ patients $=2 \mathrm{~min} /$ patient. The Takt time of $2 \mathrm{~min}$ does not mean that the patients are treated for only $2 \mathrm{~min}$ (contradicting the service times), but it is every 2 min a patient should move out of the department. If the Takt time is $<2 \mathrm{~min}$, the service in the department is faster than patient demand and the resources either wait or stay idle. If it exceeds $2 \mathrm{~min}$, then the patient waits. In order to achieve a Takt of $2 \mathrm{~min}$, the department needs $r$ resources:

$r=\frac{\text { Service time }}{\text { Takt time }}=\frac{5}{2} \approx 3$

The details of Takt time management are provided in the literature. ${ }^{[54-57]}$

The DAs collect the real-time data and alert the RSA when the waiting time in departments exceeds the threshold waiting time. This threshold value is determined to avoid unregulated waiting times in departments. The RSA identifies the number of required resources, considering constraints like consultation rooms and equipment in those departments.

Let $r_{n}$ be the number of resources in the $n^{\text {th }}$ department where $n=1, . ., N$.

$x_{n}$ be the available equipment or consultation rooms in the department.

$R_{n}$ is the total available resources for each department.

RSA calculates $r_{n}$ by using equations ( 6.1 and 6.2 ), such that: 
$r_{n} \leq x_{n}$

$r_{n} \leq R_{n}$

$r_{\mathrm{n}}$ integer

Inequality (6.3) requires that allocated resources be within the available equipment or consultation rooms. Inequality (6.4) is satisfied when the allocation for each department is within the available resources. In addition, all allocations must be integers (6.5).

\section{Coordination Mechanism}

Now, the additional vacancy created (resources required) needs to be communicated to all RAs. Resource scheduling in real-time requires an efficient coordination mechanism. We use auction-bidding, as it improves both OPC-wide (global) interest and self-interest of bidders (local). It removes the requirement of extensive one-to-one negotiations between managers and resources. It enables comparison-based selection and fairly allocates resources to departments. We implement auction-bidding for resource scheduling through the $n$-player Bayesian game with incomplete information. The game consists of the following:

Players $i \in\{1,2, \ldots ., l\}$

A set of actions (pure strategies) for each player $a_{i} \in A_{i}$

A set of types for each player $i \in \theta_{i}$;

A payoff function (reward) for each player $R_{i,} i=1, \ldots, l$.

A probability distribution $p\left(\theta_{1}, \ldots, \theta_{1}\right)$ over types of players.

Here, in this case, the players (also bidders) are the RAs. The auction-bidding is a game between RAs where they are competing with each other to improve their utilisation. The players have certain strategies and bid for the departments with slack resources.

The RSA initialises the auction. It broadcasts the call for bids to all RAs in the units and resource pool. RA (bidder) $i=1 \ldots, l$, observes a signal and prepares its bid value $v_{i}$. Bidders are risk neutral, i.e., they are interested in maximising their utilisation. There is no real cost associated, but the price that bidders offer is identified by the RSA. A set of auction rules or mechanism design will give rise to a game between the RAs. Bidders' information and value are independent (private) from each other. In this case, bidders submit their 
current utilisation $u_{i}$ (normalised value), time of last transfer $t_{i}$, the number of transfers till the time $T R_{i}$ (at the start of the day it is 1 ) and the distance between current and required department $d_{i}$ (stored in the database). The utility function for each bidder is computed as follows.

$U F_{i}=u_{i}^{*} T R_{i}^{*} d_{i}$

With these auction rules, RAs play the game within the strategy space. Bidders submit sealed bids $b_{1}, b_{2}, . ., b_{1}$. The bidder with the lowest value wins the bid (vacancy). The winner pays his own bids in the form of reward for RSA. The goal of RSA is to maximise its rewards. Initially, the reward is set to zero, $R_{\text {old }}=0$.

While (bidders proposals arrive at RSA) do

\{

Compare all bids and select the bid with Min $U F_{i}$

\}

RSA gets rewards that are calculated on the basis of reduction in waiting time for the action of resource transfer $\left(w(t)_{\text {new }}\right)$ :

$R=\frac{w(t)_{\text {old }}-w(t)_{\text {new }}}{w(t)_{\text {old }}}$

Update reward,

$R \leftarrow R_{\text {old }}+R$

A Foundation for Intelligent Physical Agents compliant ACL message is used for agent communication. A confirmation message is sent to the winning bidder (RA) and to the related MA. The winner is transferred from the current department or from the resource pool to the required department and the database is updated. MAs monitor this transfer of resources. The selected RA then:

- Receives the message

- Updates the agent data

- $t_{i} \leftarrow t_{i}$ (new)

- $T R_{i} \leftarrow T R_{i}+1$.

If the rescheduled RA does not reach the allotted department by a predefined time (in minutes), then the RSA again calls for bids. The time taken by the real-time scheduler to 
coordinate and reschedule resources is 'response time' of the MAS. With this mechanism, both individual goals (utilisation) and global goal (waiting time) of the OPC are achieved.

\section{MATERIALS AND METHODS}

\section{Data Collection and Analysis}

The initial data was collected by interviews with hospital staff of AEH to understand the workflow. Then, the data related to process and patient were collected from IHMS and CMS from January 2012 to June 2012. The patient demand, arrival times, in-time and out-time of patients and resources, resource schedule and load distribution in both units was collected. The waiting times, cycle times, patient mix, reaction times, service times and utilisation were extracted from the collected data. The probability distribution of service time and patient arrival time was determined by a data fitting tool, called Easy-Fit. The data analysis showed that the patient arrival pattern had two peaks, at around 8:00 am and 10:00 am. Therefore, a bimodal Poisson distribution ${ }^{[58,59]}$ was selected to generate model arrival times (Equation 6.9).

$$
P=\left\{v_{1}, v_{2}\right\} \text { and } \lambda=\left\{\lambda_{1}, \lambda_{2}, P\right\}
$$

Where, $P$ is the sum of two Poisson distributions with mean arrivals $\lambda_{1}$ and $\lambda_{2}$, mixed with proportions: $v_{1}=0.35$ and $v_{2}=0.65$. The goodness of fit test for input and output distribution was conducted using the Kolmogorov-Smirnov test. The managers and the information technology (IT) department of the OPC verified the workflow of the model using flowcharts and structured walks.

\section{Model Development}

A MAS of the OPC was developed using Microsoft's .NET Framework, Visual Studio .NET 2008, SQL Server Management Studio (SSMS) database and C\# programming language. The .NET framework was chosen to create communicating agents. It provides unified sets of class libraries and built-in support for the multi-protocol request-response communication between agents. The message transport mechanism delivered messages to agents based on delegated method and publication mechanism. SSMS was chosen to store gathered and analysed data over the life-cycle of the system. Patients and resources were the entities whose progress was tracked through the OPC. The model was developed on the predefined operation logics, such as patient type, pathways, departments, resources, service times, arrival times (in-time and out-time) and reaction time. Randomly generated service times were uniformly distributed between the minimum and maximum processing times from empirical data of each department. The managers and the IT department of the OPC verified the 
model. Further, the model was calibrated by assigning the reaction time randomly between 20 and 30 min to improve the accuracy. The simulation model was run with the empirical data and the performance measures, namely, waiting times, cycle times, utilisation and load distribution, were collected. Results of the simulation model were compared with the empirical data of the OPC for validation as shown in Table 6.1 and there was no statistical difference between the two.

\section{Experimental Design}

In this study, patient and resources were scheduled depending on demand and arrival time. Patient demand in AEH is stochastic with an average of 1800 patients/day with $30.8 \%$ of the monthly patient demand being $1000-1600$ patients/day, $49.9 \%$ of it being $1600-2000$ patients/day and $19.3 \%$ of it being $>2000$ patients/day. We classified patient demand into low, medium and high. We tested two sets of scheduling rules, namely, the existing model and the real-time scheduling with coordination mechanism (MAS). When resources are scheduled in real-time, the reaction time becomes extremely important. Therefore, we select the reaction time (in minutes) as three levels: $r\left(t_{1}\right)$ that is $\leq 10,11 \leq r\left(t_{2}\right) \leq 20$ and $21 \leq$ $r\left(t_{3}\right) \leq 30 \mathrm{~min}$. Reaction times were assigned randomly to the departments in the selected range. Additionally, to analyse the effect of reaction times on departmental performance, we selected six combinations of reaction times based on service times (high and low). There were in total $2^{1} \times 3^{1} \times 9^{1}=54$ experiments, and the performance measures were recorded for all the experiments in the design. A full factorial experiment was carried out to estimate the effect of selected factors on the performance parameters.

Table 6.1: Validation of the simulation model with the existing OPC in AEH

\begin{tabular}{|c|c|c|c|c|}
\hline \multirow[t]{2}{*}{ Patient demand } & \multirow[t]{2}{*}{ Performance measures } & \multicolumn{2}{|c|}{ Mean $\pm S D$} & \multirow[t]{2}{*}{$P$ value } \\
\hline & & Existing $\mathrm{AEH}$ & Simulation model & \\
\hline Low & Waiting times in minutes & $48.6 \pm 12.5$ & $47.2 \pm 11.1$ & 0.5 \\
\hline Medium & & $68.2 \pm 18.6$ & $69.9 \pm 17.2$ & 0.4 \\
\hline High & & $82.1 \pm 25$ & $82.2 \pm 22.5$ & 0.4 \\
\hline Low & Cycle times in minutes & $98.9 \pm 14.3$ & $96.9 \pm 13.2$ & 0.5 \\
\hline Medium & & $122.3 \pm 17.8$ & $120.7 \pm 18.1$ & 0.5 \\
\hline High & & $138.9 \pm 27.1$ & $135.9 \pm 24.9$ & 0.4 \\
\hline Low & Load distribution in number of patients & $3.3 \pm 1.2$ & $1.7 \pm 0.9$ & 0.4 \\
\hline Medium & & $4.1 \pm 2.5$ & $2.4 \pm 1.2$ & 0.5 \\
\hline High & & $4.3 \pm 2.8$ & $3.9 \pm 1.6$ & 0.5 \\
\hline Low & Resource utilisation in percentage & $76 \pm 3.2$ & $73 \pm 2.1$ & 0.4 \\
\hline Medium & & $78 \pm 2.4$ & $76 \pm 2$ & 0.6 \\
\hline High & & $82 \pm 2.2$ & $80.9 \pm 3.1$ & 0.5 \\
\hline
\end{tabular}

SD: Standard deviation, OPC: Outpatient clinic, AEH: Aravind Eye Hospital 


\section{Simulation Runs}

The experimental design has been replicated 10 times with 540 runs. The simulation of a day took around $10 \mathrm{~min} /$ day. The seed in random variate was varied to generate different arrival times for the same mean patient arrivals. The same randomiser input was used for simulation with two different scheduling scenarios (existing and real-time) to assure that the results obtained were not due to different inputs. The mean and standard deviation of the waiting times, cycle times, load distribution and resource utilisation were collected. These output results were analysed and compared with those from the existing model. ANOVA-tests were conducted for statistical comparisons at a significance level of 0.05. Additionally, ANOVA-tests were performed using Minitab to determine the significance of main effects and interaction effects of proposed (real-time) scheduling and reaction time on waiting times. Additionally, effects of resource scheduling and patient scheduling were analysed independently as well as together.

\section{Implementation of the Real-Time Scheduling Model in AEH}

The proposed scheduling model was implemented in the OPC during April 2014 and May 2014. The scheduling algorithms were integrated into the IHMS. Figure 6.5 shows the timing and interaction of data during real-time scheduling in the IHMS. After a patient arrives at

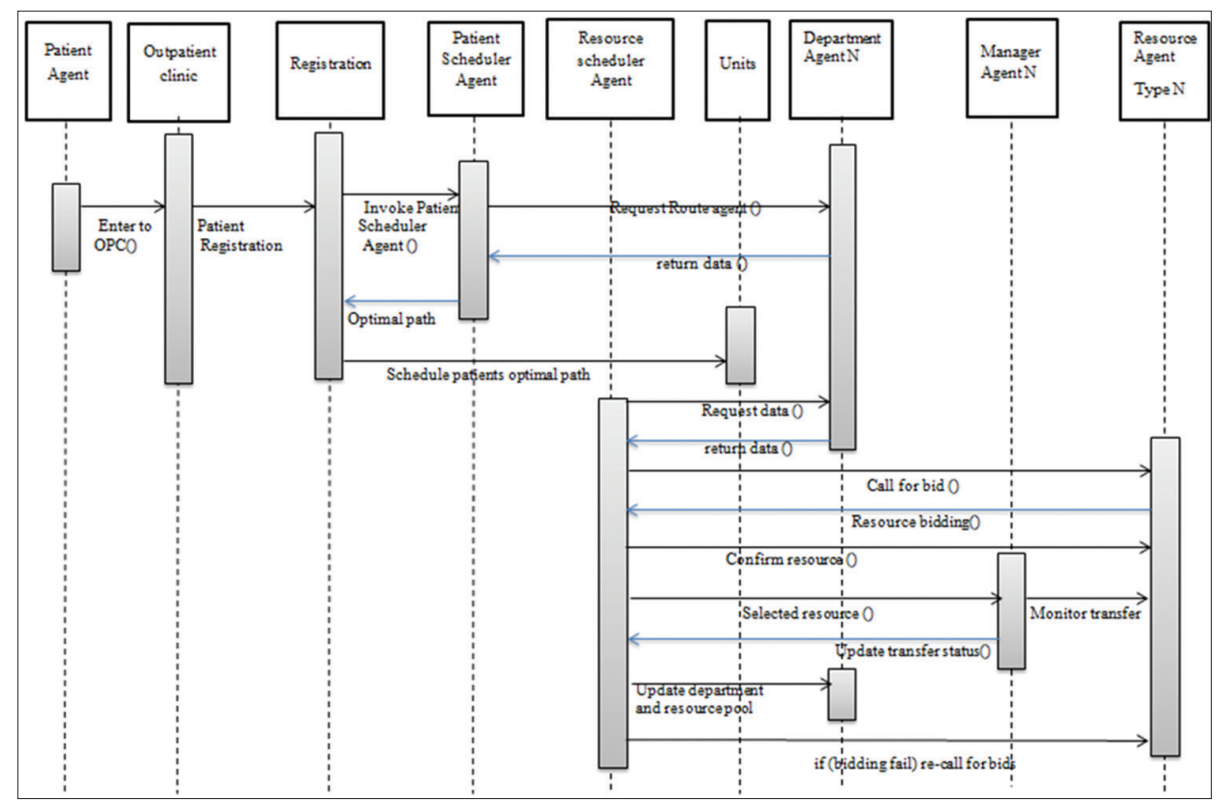

Figure 6.5: Timing and interaction of data during real-time coordination and scheduling in Integrated Hospital Management System 
the OPC, a staff member enters the patient details in a form at the registration department and invokes the scheduler. Further, the patient scheduler requests real-time data from the database. The database extracts real-time data from all departments and sends it to the scheduler. The patient scheduler finds the optimal path and prints it on the patient information card.

The resource scheduler was implemented in the OPC using the SQL PHP server, Android mobile and database server as shown. The auction and bidding were managed by an Android application developed to pull data every 30 s from a database (MySQL) via a PHP web-service (communication mode) and send SMS. An Android device was connected through a Wi-Fi connection, using a free SMS pack plan for cost effectiveness. The polling collected the information of the waiting patients in the department. This enabled the auction procedure. The call for the auction was sent to the RAs via the Android device. The RAs were provided with mobile phones to bid. This auction-bid communication was performed via web services. The database was updated after the selection of RA. The performance measures were collected from IHMS and CMS. ANOVA-tests for simulation and implementation results were conducted for statistical comparisons at a significance level of $0.05 P$ value.

\section{RESULTS}

We report the results of simulation followed by implementation of the proposed real-time scheduling model. In the simulation, the mean cycle time for the existing scheduling and real-time scheduling scheme was $120 \pm 19.7 \mathrm{~min}$ and $83.3 \pm 6.7 \mathrm{~min}$, respectively. The mean waiting time was $66.3 \pm 18.7 \mathrm{~min}$ and $29.3 \pm 9.3 \mathrm{~min}$, respectively. The waiting time reduced by $56 \%$ by using the real-time resource and patient scheduling. Statistical measures like mean and standard deviation for the waiting time, cycle time and resource utilisation for the existing versus the real-time scheme are compared in Tables 6.2-6.4. ANOVA-tests $(P=0.05)$ show that performance measures of real-time scheduling are significantly better than the existing scheduling scheme.

Table 6.2: Comparison of waiting time in minutes of the existing and the real-time scheduling

\begin{tabular}{|c|c|c|c|c|c|c|c|c|c|}
\hline \multirow{3}{*}{$\begin{array}{l}\text { Patient } \\
\text { demand }\end{array}$} & \multicolumn{3}{|c|}{$r(t 1) \min$} & \multicolumn{3}{|c|}{$r(t 2) \min$} & \multicolumn{3}{|c|}{$r(t 3) \min$} \\
\hline & \multicolumn{2}{|c|}{ Mean $\pm S D$} & \multirow[t]{2}{*}{$P$ value } & \multicolumn{2}{|c|}{ Mean $\pm S D$} & \multirow[t]{2}{*}{$P$ value } & \multicolumn{2}{|c|}{ Mean $\pm S D$} & \multirow[t]{2}{*}{$P$ value } \\
\hline & $\begin{array}{l}\text { Existing } \\
\text { scheduling } \\
\text { scheme }\end{array}$ & $\begin{array}{c}\text { Real-time } \\
\text { scheduling } \\
\text { (MAS) }\end{array}$ & & $\begin{array}{l}\text { Existing } \\
\text { scheduling } \\
\text { scheme }\end{array}$ & $\begin{array}{c}\text { Real-time } \\
\text { scheduling } \\
\text { (MAS) }\end{array}$ & & $\begin{array}{l}\text { Existing } \\
\text { scheduling } \\
\text { scheme }\end{array}$ & $\begin{array}{c}\text { Real-time } \\
\text { scheduling } \\
\text { (MAS) }\end{array}$ & \\
\hline Low & $44.9 \pm 9.6$ & $12.4 \pm 6.8$ & 0.001 & $45.3 \pm 10.2$ & $20.33 \pm 7.9$ & 0.001 & $50.9 \pm 11.2$ & $30.6 \pm 9.1$ & 0.003 \\
\hline Medium & $57.8 \pm 14.3$ & $16.9 \pm 6.3$ & 0.002 & $66.1 \pm 15.9$ & $30.6 \pm 8.9$ & 0.003 & $70.1 \pm 18.9$ & $40.1 \pm 11.7$ & 0.004 \\
\hline High & $70.1 \pm 19.4$ & $22.4 \pm 7.1$ & 0.004 & $78.9 \pm 20.1$ & $37.7 \pm 9.9$ & 0.004 & $87.1 \pm 23.6$ & $53.4 \pm 15.2$ & 0.006 \\
\hline
\end{tabular}

SD: Standard deviation, MAS: Multi-agent systems 


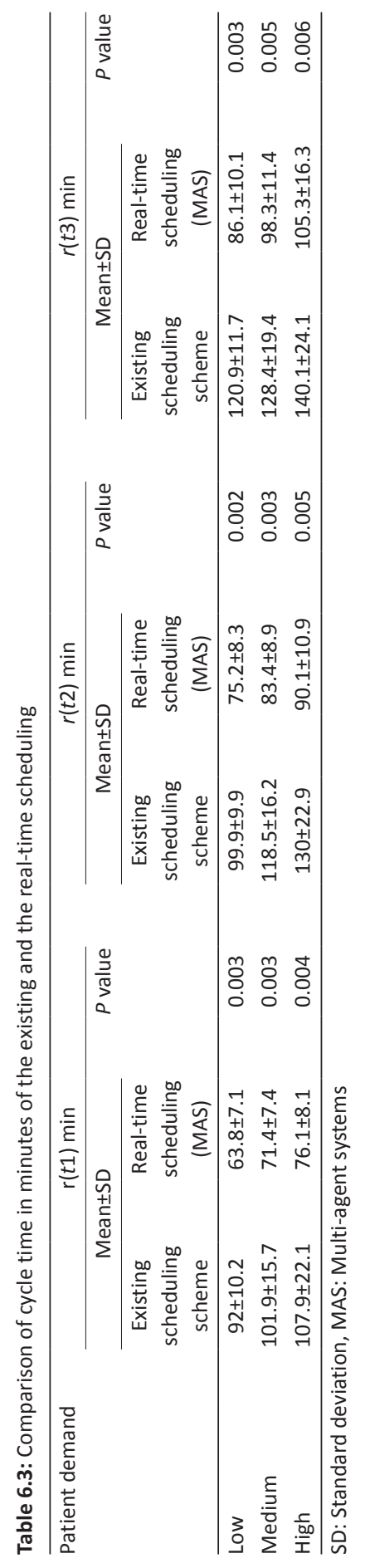


Table 6.4: Comparison of resource utilisation in percentage of the existing and the real-time scheduling

\begin{tabular}{|c|c|c|c|c|c|c|c|c|c|}
\hline \multirow{3}{*}{$\begin{array}{l}\text { Patient } \\
\text { demand }\end{array}$} & \multicolumn{3}{|c|}{$r(t 1) \min$} & \multicolumn{3}{|c|}{$r(t 2) \min$} & \multicolumn{3}{|c|}{$r(t 3) \min$} \\
\hline & \multicolumn{2}{|c|}{ Mean $\pm S D$} & \multirow[t]{2}{*}{$P$ value } & \multicolumn{2}{|c|}{ Mean $\pm S D$} & \multirow[t]{2}{*}{$P$ value } & \multicolumn{2}{|c|}{ Mean $\pm S D$} & \multirow[t]{2}{*}{$P$ value } \\
\hline & $\begin{array}{l}\text { Existing } \\
\text { scheduling } \\
\text { scheme }\end{array}$ & $\begin{array}{c}\text { Real-time } \\
\text { scheduling } \\
\text { (MAS) }\end{array}$ & & $\begin{array}{l}\text { Existing } \\
\text { scheduling } \\
\text { scheme }\end{array}$ & $\begin{array}{l}\text { Real-time } \\
\text { scheduling } \\
\text { (MAS) }\end{array}$ & & $\begin{array}{l}\text { Existing } \\
\text { scheduling } \\
\text { scheme }\end{array}$ & $\begin{array}{c}\text { Real-time } \\
\text { scheduling } \\
\text { (MAS) }\end{array}$ & \\
\hline Low & $75.1 \pm 1.6$ & $89.7 \pm 0.9$ & 0.007 & $74.2 \pm 1.8$ & $85.8 \pm 1.7$ & 0.003 & $68.4 \pm 1.9$ & $80.1 \pm 1.9$ & 0.004 \\
\hline Medium & $77.1 \pm 1.9$ & $93.8 \pm 1.1$ & 0.004 & $75.4 \pm 2.1$ & $89.4 \pm 1.9$ & 0.003 & $71.1 \pm 2.1$ & $81.1 \pm 2.2$ & 0.004 \\
\hline High & $78.2 \pm 2.1$ & $95.6 \pm 1.0$ & 0.004 & $77.3 \pm 3.1$ & $93.7 \pm 2.1$ & 0.005 & $69.2 \pm 3.3$ & $86.4 \pm 2.8$ & 0.007 \\
\hline
\end{tabular}

SD: Standard deviation, MAS: Multi-agent systems

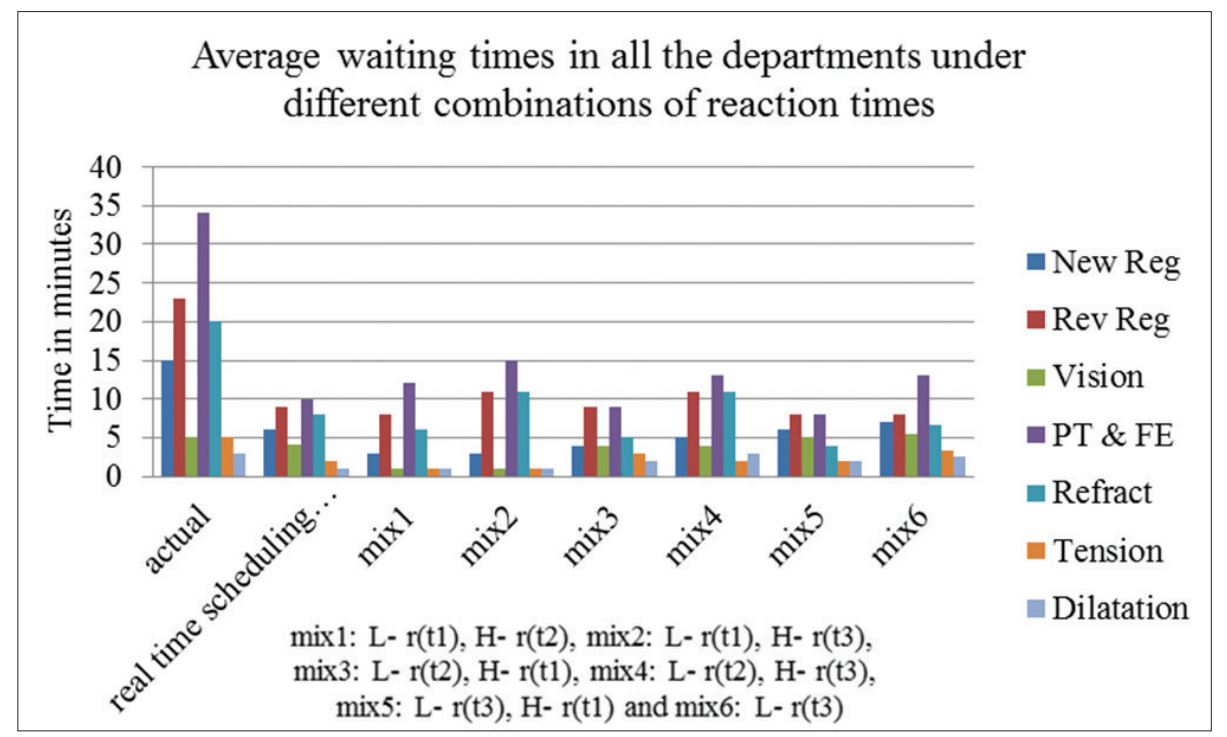

Figure 6.6: Effect of reaction times on average waiting times in all departments

The average waiting times in all departments were reduced (Figure 6.6). The figure compares the waiting times in departments for various combinations of different reaction times. Reaction times were selected on the basis of low and high service times. For example, in mix1, departments that have low $(\mathrm{L})$ service times $\left(<5 \mathrm{~min}\right.$ ) have reaction times $r\left(t_{1}\right)$ and departments with high $(H)$ service times have reaction times $r\left(t_{2}\right)$. Similarly, in mix 2, departments with low $(\mathrm{L})$ service times have reaction times $r\left(t_{1}\right)$ and departments with high $(\mathrm{H})$ service times have reaction times $r\left(t_{3}\right)$ and so on.

The two-way ANOVA-tests $(P=0.05)$ showed the significance of main effects and interaction effects of real-time scheduling and reaction time: Real-time scheduling (0.003), reaction time (0.072) and real-time scheduling*reaction time (0.039). This shows that the main effect of scheduling on waiting times is significant whereas the main effect of reaction 
time is not significant. However, the interaction effects of scheduling and reaction time are significant.

As reaction times between departments varied, tests of effects between subjects were

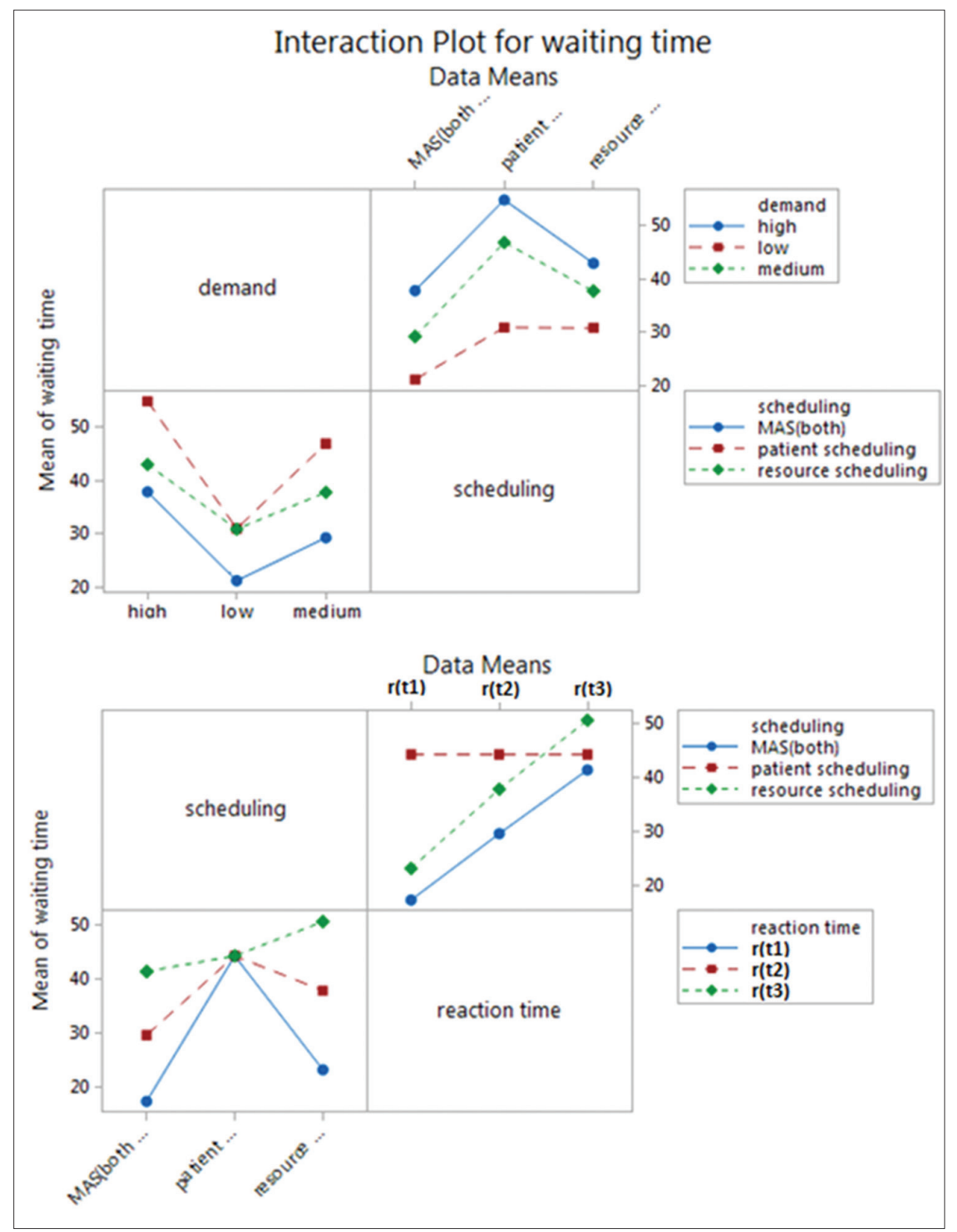

Figure 6.7: Interaction effects of resource scheduling, patient scheduling and resource coordination 
conducted. Main and interaction effects of departments, scheduling and reaction times on performance measures were analysed (with $P$ values for department*scheduling [0.001], department*reaction time [0.50] and scheduling*reaction time [0.003]). We observe that main and interaction effects of scheduling are significant in all departments whereas reaction time is not significant in departments with shorter service times. Interaction effects of resource scheduling, patient scheduling and real-time scheduling (resource + patient) are compared in Figure 6.7.

The results before and after implementation of real-time scheduling are compared in Table 6.5. There was a significant difference in performance measures between the current scheduling scheme and the real-time scheduling model. The waiting time reduced from 66.3 \pm 18.7 to $32.1 \pm 9.0 \mathrm{~min}$ and the cycle time reduced from $120 \pm 19.7$ to $84.9 \pm 11.6 \mathrm{~min}$, when real-time coordination mechanism was used in scheduling resources. In addition, resource utilisation was improved from $78.7 \pm 2.6 \%$ to $87.3 \pm 2.4 \%$.

The performance measures of the proposed model were significantly different from those of the existing situation. A $P=0.05$ was selected, meaning that the null hypothesis is rejected for a $P<0.05$ and the difference is statistically significant.

\section{DISCUSSION}

The OPC system consists of disparate departments that are locally controlled and resources that are scheduled on the basis of average demand, ahead of time and without real-time coordination. The OPC, like other hospitals (as seen in literature), is an open loop system and is prone to variability and uncertainty. Additionally, the current way of managing operations causes congestion resulting in long waiting times and cycle times. In this study, resources and

Table 6.5: Comparison of waiting time, cycle time and resource utilisation before and after implementation of the real-time scheduling model in the OPC (AEH)

\begin{tabular}{llccr}
\hline Performance measures & Patient demand & \multicolumn{2}{c}{ Mean \pm SD } & $P$ value \\
\cline { 3 - 4 } & & Before & After & 0.03 \\
Waiting times in minutes & Low & $48.6 \pm 12.45$ & $21.2 \pm 7.5$ & 0.02 \\
& Medium & $68.2 \pm 18.56$ & $33.4 \pm 9.3$ & 0.01 \\
& High & $82.1 \pm 25.02$ & $41.9 \pm 10.3$ & 0.02 \\
Cycle times in minutes & Low & $98.9 \pm 14.25$ & $70.9 \pm 9.4$ & 0.01 \\
& Medium & $122.3 \pm 17.83$ & $85.9 \pm 14.5$ & 0.01 \\
Resource utilisation in percentage & High & $138.9 \pm 27.12$ & $98 \pm 10.9$ & 0.03 \\
& Low & $76 \pm 3.2$ & $83.5 \pm 2.1$ & 0.04 \\
& Medium & $78 \pm 2.4$ & $85.2 \pm 2.4$ & 0.03 \\
\hline
\end{tabular}

SD: Standard deviation, OPC: Outpatient clinic, AEH: Aravind Eye Hospital 
patients are scheduled in real-time. For real-time scheduling, a real-time coordination mechanism is used. The scheduling has been performed in two facets: Real-time (actual) demand and in global perspective. Our findings confirm that real-time scheduling reduced the average waiting time by $56 \%$ (simulation) and by $51.6 \%$ (implementation). It improved resource utilisation by $8.3 \%$ with imposed constraints during implementation. The study demonstrates the effects of patient demand, scheduling rules and reaction time on waiting times, cycle times and resource utilisation. It was found that global control of operations and real-time scheduling improves performance measures, such as waiting times and cycle times, compared to the current method of scheduling. The agents in the OPC represented a bad game where only individual goals were achieved. The Bayesian game played through coordination mechanism based on the actual status of departments: Auction-bidding procedure improved the fulfilment of individual goals as well as OPC-wide goals.

Besides scheduling rules and control of operations, reaction time influences waiting times in the OPC. ANOVA was used to determine the main and interaction effects of real-time scheduling and reaction time. Effects of real-time scheduling are significant on performance measures like waiting times, cycle times and utilisation. It shows improvement in reaction time does not improve waiting times on its own but has a significant effect on performance measures when implemented along with real-time scheduling. Further, the greater reaction times in departments with longer service times affect the performance measures when compared to shorter reaction times in departments with smaller service times.

Real-time scheduling, as studied here, used resource scheduling and patient scheduling simultaneously. The two scheduling methods perform well individually, but together as MAS, they perform even better (Figure 6.7). Patients were scheduled to the pathways with minimum waiting times at the registration by considering the complete pathway. The patient scheduler did not reduce the waiting times at registration departments. The resources in these departments were rescheduled on the basis of the demand by real-time resource scheduling.

The MAS model has real-time coordination with resource pooling and proactive bidding of resources. This improved the resource utilisation. AEH has dedicated staff who proactively participated in resource bidding. The resources were scheduled to the departments throughout the OPC system and the managers monitored them. The resources were matched to the varying patient demand in real-time. The MAS modelling of the OPC allowed harmonising the performance of the OPC with conflicting goals of the agents (resources, patients and management). Coordination and information sharing between agents improved OPC-wide performance. Delays in decision-making due to management hierarchy were reduced, as decisions were taken on the basis of real-time status and information of the departments. 
The literature shows that planning often fails to incorporate short-term variabilities and uncertainties due to arrivals and service times. Previously, MAS has been used for patient scheduling and resource scheduling, but not simultaneously for both in real-time. The model in this study captures this stochasticity in walk-in demand, coordinates between departments and schedules the resources and patients in real-time. It acts like a self-analysing and self-correcting network, which incorporates the slightest change in the OPC and effectively manages its patients and resources.

The number of interactions increases with the number of active agents. Patients were passive agents; hence, an increase in the patient demand did not affect the load on the network. The resources were active agents, who communicated and negotiated to achieve their goals. The response time of the real-time scheduling model increases exponentially when there is an increase in the number of pathways, hospital departments (50 or 100) or resources. Therefore, to extend this model to other types of clinics or hospitals, the reaction time as well as the response time should be further studied. The pathways in specialised OPCs are more deterministic and less variable than one would expect in general hospitals. General hospitals have a more complex management hierarchy and more resource types than specialised OPCs. Our model can be applied to a general hospital model if response time, reaction time and patient pathways can be studied in detail. The outcome of this model in other situations is dependent on the frequency of real-time data collection based on the hospital information system.

Limitations of this implementation are the cost involved for the database servers and the main (hospital) server, and the limited accessibility (narrow area) of the network. In order to overcome the latter, data can be stored in a common place (the cloud) where all application program interfaces are clients. These communicate with each other via Service Oriented Architecture Protocol and use Web Service Definition Language as the communication language between them. The patient and process data were updated by hospital staff in the departments. As future work, we intend to utilise radio frequency identification tags for patients to update patient and process data, and to identify the resource location.

Healthcare settings like OPCs or hospitals differ in their complexity, patient groups and processes. Therefore, requirements for scheduling and control might vary. The variability and uncertainty in OPCs are not deterministic and need optimisation in a short-term. However, how we use real-time scheduling system might not only depend on the algorithm, but also on other factors, such as organisation of the OPC, the knowledge management system, crossskills of the staff (to facilitate transfer of staff from one department to the other) and the layout of the building (distances between departments). As future work, we intend to explore the real-time scheduling model in complex hospitals with stochastic pathways. 
Scheduling of resources as well as patients have been researched extensively in the recent past but has not been performed in real-time, and coordination in real-time has not been analysed. This study incorporated actual demand, service times, uncertainty, pathways, reaction times and global perspective in scheduling to optimise operations (workflow management) in real-time. This makes it possible for OPCs to provide services when the patient asks for it (pull).

Both global, as well as local/departmental goals, are achieved with the real-time scheduling model.

\section{CONCLUSION}

This study shows how real-time scheduling improves waiting times and cycle times in OPCs. The study incorporates two facets of scheduling: Real-time and global perspective. Real- time scheduling is based on actual demand and coordinates in real-time to reschedule resources. It schedules patients to the optimal pathway depending on the actual status of the departments in the OPC. Global scheduling overcomes the issues of unregulated waiting times. Reaction time, an exogenous variable, has an impact on performance measures in open loop systems like OPCs. The study shows MAS fits in OPC systems where decision-making is complex and involve many people. It provides a platform for analysing coordination mechanisms in operations control. Real-time information about departments, patients and resources provide insights in decision-making. This study shows that both departmental (local) and OPC-wide (global) performances improve by realtime scheduling, which is based on the system status of all departments in an OPC. This model has been implemented to an eye care OPC and can be extended to general hospitals by considering their demand, pathways, resources, organisational structures and workflow complexities.

\section{REFERENCES}

1. Huang XM. Patient attitude towards waiting in an outpatient clinic and its applications. Health Serv Manage Res 1994;7(1):2-8.

2. Zhu Z, Heng BH, Teow KL. Analysis of factors causing long patient waiting time and clinic overtime in outpatient clinics. J Med Syst 2012;36:707-13.

3. Khanna S, Boyle J, Good N, Lind J, Zeitz K. Time based clustering for analyzing acute hospital patient flow. In: Engineering in Medicine and Biology Society (EMBC). Annual International Conference of the IEEE. IEEE; 2012.

4. Plasters CL, Seagull FJ, Xiao Y. Coordination challenges in operating-room management: An in-depth field study. In: AMIA Annual Symposium Proceedings. Washington, DC; 2003.

5. Khanna S, Cleaver T, Sattar A, Hansen D, Stantic B. Multiagent based scheduling of elective surgery. In: Principles and Practice of Multi-Agent Systems. Berlin, Heidelberg: Springer; 2012. p. 74-89.

6. Cayirli T, Veral E. Outpatient scheduling in health care: A review of literature. Prod Oper Manag 2003;12(4):519-49.

7. McKee M, Waghorn A. Why is it so difficult to organise an outpatient clinic? J Health Serv Res Policy 2000;5(3):140-7.

8. van Merode GG, Groothuis S, Hasman A. Enterprise resource planning for hospitals. Int J Med Inform 2004;73(6):493-501. 
9. Ludwig M, van Merode F, Groot W. Principal agent relationships and the efficiency of hospitals. Eur J Health Econ 2010;11(3):291-304.

10. Vissers JM,Bertrand JW, de Vries G. A framework for production control in health care organizations. Prod Plan Control Manage Oper 2001;12(6):591-604.

11. Mansdorf BD. Allocation of resources for ambulatory care-A staffing model for outpatient clinics. Public Health Rep 1975;90(5):393-401.

12. Yurko LC, Coffee TL, Fusilero J, Yowler CJ, Brandt CP, Fratianne RB. Management of an inpatient-outpatient clinic an eight-year review. J Burn Care Rehabil 2001;22(3):250-4.

13. Ren $\mathrm{Y}$, Kiesler S, Fussell SR. Multiple group coordination in complex and dynamic task environments: Interruptions, coping mechanisms, and technology recommendations. J Manag Inf Syst 2008;25(1):105-30.

14. Güler MG. A hierarchical goal programming model for scheduling the outpatient clinics. Expert Syst Appl 2013;40(12):4906-14.

15. van Merode F. A prelude of the 2004 antwerp quality conference: Targets and target values-integrating quality management and costing. Accredit Qual Assur 2004;9(3):168-71.

16. Hulshof PJ, Mes MR, Boucherie RJ, Hans EW. Tactical Planning in Healthcare using Approximate Dynamic Programming. Memorandum 2014, Department of Applied Mathematics, University of Twente, the Netherlands; 2014.

17. Harper PR. A framework for operational modelling of hospital resources. Health Care Manage Sci 2002;5(3):165-73.

18. van der Voort MM, van Merode FG, Berden BH. Making sense of delays in outpatient specialty care: A system perspective. Health Policy 2010;97(1):44-52.

19. Vermeulen IB, Bohte SM, Elkhuizen SG, Lameris H, Bakker PJ, La Poutré H. Adaptive resource allocation for efficient patient scheduling. Artif Intell Med 2009;46(1):67-80.

20. Holleman DR, Bowling RL, Gathy C. Predicting daily visits to a walk-in clinic and emergency department using calendar and weather data. J Gen Intern Med 1996;11(4):37-9.

21. Cayirli T, Gunes ED. Outpatient appointment scheduling in presence of seasonal walk-ins. J Oper Res Soc 2014;65(4):512-31.

22. Peng Y, Qu X, Shi J. A hybrid simulation and genetic algorithm approach to determine the optimal scheduling templates for open access clinics admitting walk-in patients. Comput Ind Eng 2014;72(1):282-96.

23. Su S, Shih CL. Managing a mixed-registration-type appointment system in outpatient clinics. Int J Med Inform 2003;70(1):31-40.

24. BarilC, Gascon V, Cartier S. Design and analysis of an outpatient orthopaedic clinic performance with discrete event simulation and design of experiments. Comput Ind Eng 2014;78:285-98.

25. Yang X, Han R, Guo Y, Bradley J, Cox B, Dickinson R, et al. Modelling and performance analysis of clinical pathways using the stochastic process algebra PEPA. BMC Bioinform 2012;13(14):1-17.

26. Schrijvers G, van Hoorn A, Huiskes N. The care pathway: Concepts and theories: An introduction. Int J Integr Care 2012;12:e192.

27. Hossain L, Guan DC. Modelling coordination in hospital emergency departments through social network analysis. Disasters 2012;36(2):338-64.

28. Gloede TD, Hammer A, Ommen O, Ernstmann N, Pfaff H. Is social capital as perceived by the medical director associated with coordination among hospital staff? A nationwide survey in German hospitals. J Interprof Care 2013;27(2):171-6.

29. Mors AT, Yadati C, Witteveen C, Zhang Y. Coordination by design and the price of autonomy. Auton Agent Multi Agent Syst 2010;20:308-41.

30. Bogg P, Beydoun G, Low G. When to use a multi-agent systems? In: Intelligent Agents and Multi-Agent Systems. Berlin, Heidelberg: Springer; 2008. p. 98-108.

31. Jemal H, Kechaou Z, Ayed MB, Alimi AM. A multi agent system for hospital organization. Int J Mach Learn Comput 2015;5(1):51-6.

32. Zöller A, Braubach L, Pokahr A, Rothlauf F, Paulussen TO, Lamersdorf W, et al. Evaluation of a multi-agent system for hospital patient scheduling. Int Trans Syst Sci Appl 2006;1(4):375-80. 
33. Paulussen TO, Zöller A, Heinzl A, Pokahr A, Braubach L, Lamersdorf W. Dynamic patient scheduling in hospitals. Coordination and Agent Technology in Value Networks. Berlin: GITO; 2004. p. 149-74.

34. Paulussen TO, Jennings NR, Decker KS, Heinzl A. Distributed patient scheduling in hospitals. In: Proceedings of the Eighteenth International Joint Conference on Artificial Intelligence (IJCAI-03). Morgan Kaufmann; 2003.

35. Huang J, Han Z, Chiang M, Poor HV. Auction-based resource allocation for cooperative communications. IEEE J Sel Areas Commun 2008;26(7):1226-37.

36. Tipsuwan Y, Kamonsantiroj S, Srisabye J, Chongstitvattana P. An auction-based dynamic bandwidth allocation with sensitivity in a wireless networked control system. Comput Ind Eng 2009;57:114-24.

37. Jonker CM, Robu V, Treur J. An agent architecture for multi-attribute negotiation using incomplete preference information. Auton Agent Multi Agent Syst 2007;15:221-52.

38. Pendharkar PC. Game theoretical applications for multi-agent systems. Exp Syst Appl 2012;39:273-9.

39. Štiglic G, Kokol P. Intelligent patient and nurse scheduling in ambulatory health care centers. In: Engineering in Medicine and Biology $27^{\text {th }}$ Annual Conference. Shanghai, China; 2005.

40. Williamson M, Decker KS, Sycara K. Executing decision-theoretic plans in multi-agent environments. In: AAAI Fall Symposium on Plan Execution. Cambridge, Massachusetts: AAAI Press; 1996.

41. Decker KS. Distributed problem-solving techniques: A survey. IEEE Trans Syst Man Cybern 1987;17(5):729-40.

42. Deshpande U, Gupta A, Basu A. A distributed hospital resource scheduling system using a multi-agent framework. IETE Tech Rev 2001;18(4):263-75.

43. Semsar-Kazerooni E, Khorasani K. Multi-agent team cooperation: A game theory approach. Automatica 2009;45:2205-13.

44. Brilliant L, Brilliant G. Aravind: Partner and social science innovator (innovations case discussion: Aravind eye care system). Innov Technol Gov Glob 2007;2(4):50-2.

45. Mehta PK, Shenoy S. Infinite Vision-How Aravind Became The Greatest Business Case for Compassion. San Franciso: Berrett-Koehler Inc.; 2011. p. 336.

46. Chaudhary B, Modi AG, Reddy K. Right to sight: A management case study on Aravind Eye Hospitals. ZENITH Int J Multidiscip Res 2012;2(1):447-57.

47. Aravind Eye Care System: Activity Report, 2014-15: Madurai. p. 76.

48. Rangan VK, Thulasiraj RD. Making sight affordable (innovations case narrative: The Aravind Eye Care System). Innov Technol Gov Glob Fall 2007;2(4):35-49.

49. Andersen MM, Poulfelt F. Beyond Strategy: The Impact of Next Generation Companies. New York: Routledge; 2014.

50. Natchiar G, Thulasiraj RD, Sundaram RM. Cataract surgery at Aravind Eye Hospitals: 1988-2008. Commun Eye Health 2008;21(67):40-2.

51. Khanna S, Sattar A, Maeder A, Stantic B. Intelligent Scheduling in Complex Dynamic Distributed Environments. Medinfo 2007: Proceedings of the $12^{\text {th }}$ World Congress on Health (Medical) Informatics; Building Sustainable Health Systems; 2007. p. 1665.

52. Gambardella LM, Dorigo M. An ant colony system hybridized with a new local search for the sequential ordering problem. INFORMS J Comput 2000;12(3):237-55.

53. Dorigo M, Gambardella LM. Ant colony system: A cooperative learning approach to the traveling salesman problem. IEEE Trans Evol Comput 1997;1(1):53-66.

54. Liker JK. The Toyota Way: 14 Management Principles from the World's Greatest Manufacturer. New York: McGraw-Hill; 2004. p. 330.

55. Hopp WJ, Spearman ML. Factory Physics-Foundations of Manufacturing Management. New York, NY:Irwin, McGraw-Hill; 2001. p. 698.

56. Eswaramoorthi M, Kathiresan GR, Jayasudhan TJ, Prasad PS, Mohanram PV. Flow index based line balancing: A tool to improve the leanness of assembly line design. Int J Prod Res 2012;50(12):3345-58.

57. Smith JM, Tan B. International series in operations research and management science. Handbook of Stochastic Models and Analysis of Manufacturing System Operations. Vol. 192. New York: Springer-Verlag; 2013. p. 373.

58. Li J, Zha H. Two-way poisson mixture models for simultaneous document classification and word clustering. Comput Stat Data Anal 2006;50(1):163-80.

59. Karlis D, Xekalaki E. Mixed poisson distributions. Int Stat Rev 2005;73:35-58. 


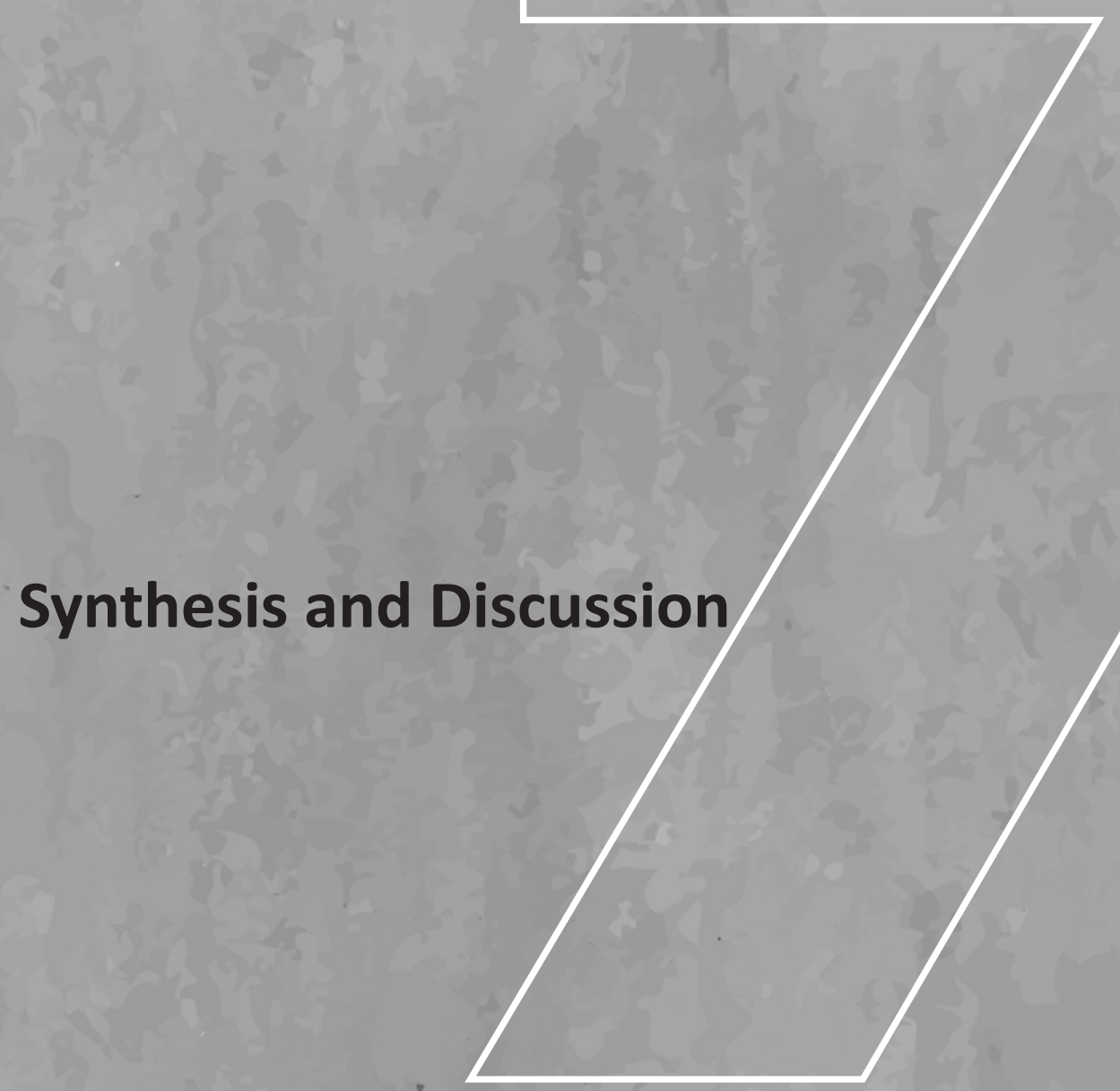





\section{INTRODUCTION}

The overall objective of this thesis is to extend knowledge concerning hospital operating systems. This thesis tests the hypothesis that "a hospital system with disparate subsystems cannot minimise cycle/waiting time by separately optimising subsystems, or by scheduling that does not adjust in real-time."

Patients usually wait for long periods before they get the required care in a hospital. A patient arriving in pain and grief expects quality and timely care. ${ }^{[1,2]}$ Prolonged waiting is not only unpleasant and redundant but can also have adverse effects on the patient and their attendants, as chances of acquiring infections from hospital increase. It is common to see patients struggling and arguing to get ahead of the queues, leading to increased stress on the patients as well as the hospital staff managing them. From the hospital perspective, it is important to increase efficiency and distribute the workload uniformly across equally skilled human resources.

Hospital managements are getting more inclined towards improving and redesigning their care delivery and are focusing on waiting and cycle times, spurred by the increasing national and international attention and by a felt need. ${ }^{[3]}$ Although several operation system methodologies have been adapted from the industry for solving these issues, they have not been adequate to address the problem efficiently.

There are several reasons why various applications of the operating systems have not yet worked optimally, and we continue to face challenges in the real world. The outpatient clinics (OPCs), with larger latency in their operations, are designed to deliver care mostly as a push system, where the capacity for care is delivered on the basis of projected average demand. Care is provided whenever the resources are ready and not when the patient needs it. ${ }^{[1]}$ Although operating systems have been developed looking at this projected demand based on appointment systems, they have not been able to address the problem adequately. In practice, even an OPC with only scheduled patients through appointments faces problems, due to the early or late arrival of patients or doctors, no-show of patients and cancelled appointments. Moreover, in many instances, especially in India, OPCs have high levels of walk-ins and systems that have been designed for appointment-based projected demand fail. Even if some appointments are given, OPCs cater to walk-ins as well, thus functioning as a mixed system. The walk-ins and mixed systems make OPCs function more like a pull system or a combination of push and pull, undermining the impact of the systems designed as a push system. Here we use the terms push and pull to differentiate only at a conceptual level ${ }^{[4]}$ and do not delve into the varying definitions used by different authors.

In the automobile industry, some successful models use "Just In Time" (JIT) system championed by Toyota Production Systems, which is a combination of push and pull mechanisms. 
JIT mechanism works well when it is applied to the entire system and across departments. Although JIT has been tried in health care, it has been sub-optimal. This was because OPCs are functionally organised in general, the departments are managed locally and the application of operating system design is done at an individual department level. Planning and control are local to the departments, hence every department controls its patient flow for optimising its capacity usage and improving its efficiency. The departmental decisions are independently taken by managers to achieve department-specific goals, with limited knowledge ${ }^{[5-7]}$ about workflows and processes of other departments. Moreover, as the processes are interdependent, patients often visit (and re-visit) multiple departments. ${ }^{[8,9]}$ The local control and lack of coordination results in faster upstream departments pushing patients to slower downstream departments, resulting in patients waiting for care. When upstream departments are slower than downstream departments, the latter waits for work, resulting in under-utilisation of resources. ${ }^{[10]}$ Since there is no global approach that takes the entire OPC as a unit, increasing the efficiency of individual departments does not necessarily improve the overall efficiency of the OPC..$^{[7,11-13]}$

Unlike the situation in other industries, the OPCs experience high variability and uncertainty of patient demand which makes the direct use of existing methods of operation system challenging. High walk-ins, appointment cancellations, unpredictable patient conditions resulting in patient-to-patient variations in service times, the number of departments visited by the patient and complex patient pathways lead to variability and uncertainty. The ageing population with multiple comorbidities, the delayed care seeking behaviour of the population, human resource shortages, lack of education and knowledge of patients, hierarchical subjective management systems, etc. make the entire system highly complex and increase the variability and uncertainty to a further extent. Such variability and uncertainty necessitate the OPCs to be very dynamic and responsive.

However, OPCs often collect feedback after the event on the basis of which planning and scheduling are carried out in longer time horizons, usually in weeks to months and occasionally in days. Action taken through planning and scheduling at a later point cannot solve the current workflow problems when cause and effect are experienced at the same time. The OPC is an open loop system ${ }^{[14]}$ that is affected by variability and uncertainty caused due to the delay in utilising the feedback and taking action to improve the workflow. Aggregate planning does not capture short-term variability in demand in OPCs. ${ }^{[15]}$ While scheduling in OPCs is extensively studied, real-time scheduling remains understudied. Being responsive to a changing demand can be achieved by accommodating changes in real-time.

As the current OPC workflow management methods are not demand-driven and are not optimised either globally or in real-time, they do not always accomplish the desired outcomes. In order to achieve the goal of reduced waiting and cycle time, it is hypothesised that OPCS 
should not only plan, schedule and control operations in real-time but should also be optimised globally as a system.

\section{METHODS}

\section{Research Hypothesis}

We adopt a stepwise approach to addressing this hypothesis. The main research question being, "Does overall (global) optimisation along with real-time scheduling improve the wait/ cycle time and hospital performance?" is examined with respect to each of the major subcomponents of OPC system - resource planning, patient scheduling and coordination - to attain results at each level. Since each of these sub-components function together in reality to address the main research question fully, we look at various combinations of the optimisation models built at each sub-component level are to find the combination with the best outcomes.

This research question leads to the following sub-questions, which are explored in this study.

1. Does overall (global) optimisation along with predictive (nearly real-time) resource planning improve waiting and cycle times?

2. Does overall (global) optimisation along with real-time patient scheduling improve (reduce) waiting and cycle times?

3. Does overall (global) optimisation along with real-time coordination of resources and patients improve (reduce) waiting and cycle times?

4. What combination of each optimisation models best improves (reduces) the waiting and cycle times?

\section{Additional Outcomes}

1. Does overall (global) optimisation along with real-time coordination of resources and patients improve (increase) resource utilisation?

2. Is resource shifting across departments feasible? Is resource shifting possible for all skill levels, provided similar skills of resources be grouped and shifted within the same level?

\section{Research Approach}

The overall approach to the method while answering these questions consists of the following steps (Figure 7.1). 


\section{Study Variables}

The inputs to the operating system are the independent, uncontrollable, controllable and exogenous variables and dependent variables like waiting times, cycle times and resource utilisation are the output variables (Figure 7.2).

\section{Study Setting}

In order to test our hypothesis, it was important to choose a study setting that satisfied the following criteria:

- Patient arrivals are random and independent (all walk-ins or a mix of walk-ins and appointments), leading to high variability.

- There is no control on the total number of patients coming in for service, leading to unpredictable demand.

- Patient arrival rate varies within a day, leading to high variability.

- Local control in departments.

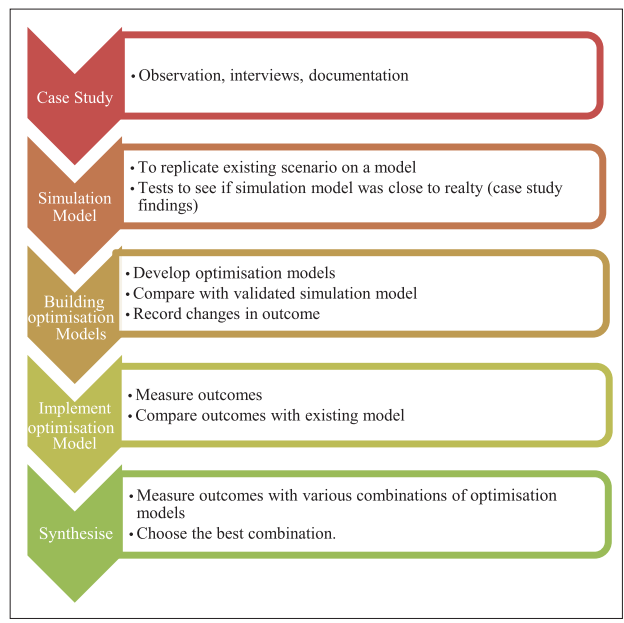

Figure 7.1: Research approach

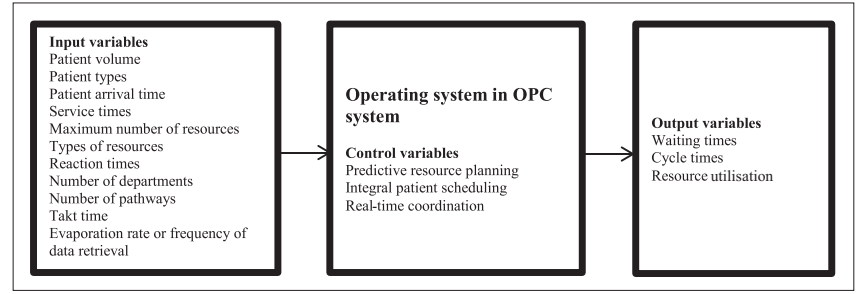

Figure 7.2 Variables in operating system of an OPC system 
- Planning is performed ahead of time, and scheduling remains fixed over long periods.

- Good IT infrastructure allowing data collection in real-time.

- Patient pathways can be predetermined with reasonable accuracy.

- Large patient load, allowing adequate sample sizes for testing the model implementation.

- Support from the hospital for any administrative changes required for implementing the model.

\section{Aravind Eye Hospital (AEH)}

AEH, Madurai (Tamil Nadu, India) was chosen for the present study as it satisfied all the criteria listed above. In AEH, all patients are walk-ins and are provided care on "first-come, first-served" basis at registration. The hospital attains its goals by strict attention to cost optimisation and high quality through its operational excellence. ${ }^{[16-19]}$ While employing $<1 \%$ of the country's ophthalmic workforce, AEH accounts for $5 \%$ of ophthalmic surgeries performed nationwide. AEH has performed 401,529 surgeries and has treated 2,396,864 outpatients during 2014-2015. ${ }^{[20]}$ It is one of the world's largest eye care service providers. ${ }^{[21,22]}$ The hospital uses a manual scheduling system and local optimisation for workflow management.

\section{Case Study and Findings}

We examined an OPC system over a period of 2 months to gain an in-depth understanding of its working environment. Patient pathways, service times for various processes and departments, workflows, managing of patients and resources, interdependencies and factors influencing waiting and cycle times were studied. This information was gathered through direct observation, interviews and sourcing of pertinent data from the integrated hospital management system and the clinical management system.

The case study revealed that resources (mainly doctors) were rescheduled to compensate for the increase in workloads in AEH. However, these modifications were under local control and were typically made through phone calls to check if anyone was available, without complete knowledge about the types of resources and patient loads in other departments. In order to address this inefficient approach to resource management, it was necessary to conduct resource coordination in a way that allows staff members with the same level of skill and expertise to be deployed in real-time, wherever necessary. We believe that this is an important contribution to the field since extensive literature review failed to reveal studies, in which a similar demand-driven resource shifting was attempted. Therefore, it is reasonable to assume that ad-hoc approaches similar to the one described above are utilised in other OPCs. 


\section{RATIONALE FOR COMBINATION MODELS}

As explained in the previous chapters, each of the optimisation models we have developed has helped in independently reducing both waiting and cycle times. Thus, the aim of this research was to design an operating system for hospitals that would satisfy patient demand with short cycle times. As the three processes (planning, scheduling and coordination) are interrelated in reality, the study intended to develop a model that allows their concurrent application.

Moreover, each model has certain limitations in isolation. For example, although the predictive resource planning is demand-driven and global, it is still at the planning level. Thus, it can only be viewed as a step towards matching resources with demand, rather than a complete solution. It gives preparedness to OPCs to handle demand variability. In practice, the actual demand needs to be handled more effectively. We observed that although the integral patient scheduling model minimises waiting times, workflows in some departments like registration remain sub-optimal. The optimisation of registration departments along with patient scheduling would further improve waiting times. When real-time scheduling is adopted, both patients and resources are scheduled efficiently with timely coordination. This model results in shorter waiting times compared to those produced by the other two models. We also noted that a higher variability mandates greater resource availability and more frequent rescheduling. The reaction time is also highly important in real-time scheduling. By incorporating resource planning, the pressure of coordinating and rescheduling would be lessened.

We presume that the impact of the inherent variability and uncertainty in an OPC system is best reduced when the optimisation models are applied jointly. Additionally, a good operating system is a combination of both push and pull. By combining the optimisation models, we can realise a right mix of both pull and push system. Therefore, we employ these three optimisation models in different combinations of the basic models as detailed below (Table 7.1).

We have dealt in detail the models that address the first three research questions in chapters 4-6 and we summarise them here. In this chapter, we deal with the fourth research question by exploring the combination of models 4,6 and 7. Finally, we present the contribution to theory and practice followed by limitations and future research.

Question 1: Does overall (global) optimisation along with predictive (nearly real-time) resource planning improve waiting and cycle times? (Model 1). 
Table 7.1: Operating models: 1-3 studied in the previous chapters, 4-7 combinations of 1-3

\begin{tabular}{ll}
\hline Models & Details of models and their combinations \\
\hline Model 1 & Predictive resource planning \\
Model 2 & Integral patient scheduling \\
Model 3 & Real-time coordination for scheduling \\
Model 4 & Predictive resource planning + Integral patient scheduling \\
Model 5 & Real-time resource scheduling \\
Model 6 & Predictive resource planning + Real-time resource scheduling \\
Model 7 & Predictive resource planning + Integral patient scheduling+ Real-time coordination for \\
& scheduling
\end{tabular}

What is known in the literature?

- Resource planning and scheduling are done on the basis of availability of resources, and it is conceptually "push."

- Long term forecasting, which is usually in the range of a day, week or month, is used for planning.

- Resources are scheduled ahead of time.

- Scheduling is always local (department-centric).

- The aim of scheduling is often cost optimisation and/or utilisation and not a reduction of waiting times.

- Takt time management is applied to systems with known demand over a timeframe of an entire day or week or month.

- Most of the time resource planning is performed to match with supply (resources) only. Although it has been matched with demand in few studies, it is limited to account for deterministic demand only.

Gaps in literature

- No demand-based (near to real-time) resource scheduling is done in hospitals/OPC.

- There is no scheduling that focuses globally across departments (entire OPC system).

- Takt time management is not applied for varying demand (stochastic demand) and a short-term of $1 \mathrm{~h}$.

- Patient-centric resource scheduling is not studied.

Optimisation model/intervention

- Resource planning model was developed on the basis of demand.

- Short-term patient demand was forecasted using previous patient arrival patterns.

- The working hours of a day were divided into time-slots of $1 \mathrm{~h}$ each.

- An optimisation model that uses Takt time was developed to find the number of resources required during each time-slots, with resources being scheduled accordingly.

- Takt time management was applied to the entire OPC system and it accounted for stochastic demand at shorter intervals of $1 \mathrm{~h}$. 
Main results

- The predictive planning model reduced the average waiting time by $43.4 \%$ during simulation (from 66.3 to $37.5 \mathrm{~min}$ ) and by $41.1 \%$ during its implementation (from 66.3 to $39.0 \mathrm{~min}$ ) in the OPC.

- The mean cycle time was reduced by $25.6 \%$ (120.1-89.3 min) during simulation and by 23.1\% during implementation (120.1-92.4 min).

- The results show that reaction time contributes to reducing waiting times by around $5 \%$ (4.89\%).

- The existing resource plan had a fixed number (28) of resources in both units in a day; whereas, the predictive resource plan has a minimum of 12 to a maximum of around 30-34 resources (only for few time-slots) in a day.

Strengths of our study

- This predictive resource planning model has been able to bring in a level of preparedness and indirectly reduce the reaction time.

- It has been demonstrated that Takt time management can also be globally applied to open loop systems, where variability is high.

- An optimisation model has been developed, which is not only tested in theory but also in implementation to see its actual effect.

- Although still being a push model, it adapts to demand.

Limitations of our study

- Though short-term variability (hour-wise) is captured, patient arrival can remain variable and uncertain to some extent within the 1-h time-slot. For example, all patients may arrive either at the beginning or at the end of the 1-h time-slot.

- The optimisation model is limited by other constraints during planning. For instance, if the required number of resources is five and there are only four consultation rooms, then the resource allocation to other departments changes accordingly, accounting for the layout constraint. Similarly, lack of equipment limits optimisation of the workflow further despite the availability of human resources. Although our optimisation model does not include these variables directly, they are accounted during the initial study and the model works with the existing limitations. However, it can quantify the improvement in waiting and cycle times that can occur with change in layout or equipment and give inputs for long-term planning. Reaction time is an exogenous variable that affects output and cannot be controlled in open loop systems. If departments have greater reaction times, then waiting times do not improve much.

From this optimisation model, we learn that it is important to not only account for shortterm variability while planning resources, but also to have a global approach for planning. Prediction of demand in the short-term helps in better preparedness and indirectly reduces 
reaction time. However, departments with higher reaction and service times are not able to show the same level of improvement compared to lower reaction and service times, prompting the need to explore other ways to overcome this limitation.

Question 2: Does overall (global) optimisation along with real-time patient scheduling improve (reduce) waiting and cycle times?

What is known in literature?

- Patient scheduling is commonly performed through appointment systems.

- Appointment systems range from manual to online. There are studies which have applied operations system research to an appointment-based system and have been able to account for variability arising out of no shows and double booking.

- However, patients are scheduled on the basis of resource availability, and the scheduling is OPC-centric. Patient scheduling is mainly a "push" mechanism.

Gaps in literature

- There is no real-time and global perspective for patient scheduling in hospitals/OPC.

- Patient pathway optimisation is not studied.

- Patient-centric systems are not studied.

Optimisation model/intervention

- An integral patient scheduling model has been developed that uses the actual system statuses of all departments in the OPC.

- Patient pathways are adapted according to the demand.

- Patients are scheduled in real time and with a global perspective.

- An ant agent algorithm has been developed by incorporating precedence constraints and has been applied for patient scheduling.

Main results

- The cycle time reduced by $18.4 \%$ (120-97.9 $\mathrm{min}$ ) and waiting time by $33 \%$ (66.3-44.2 $\mathrm{min}$ ) during simulation.

- The cycle time reduced by $12.3 \%$ (from 120 to $105 \mathrm{~min}$ ) and waiting time by $26.5 \%$ (from 66.3 to $48.7 \mathrm{~min}$ ) during implementation.

- Prior to implementation of integral patient scheduling model, $13.5 \%$ of patients spent more than $2 \mathrm{~h}$ in the OPC, which reduced to $8.7 \%$ after the implementation of the model (reduced by $35.5 \%$ ).

\section{Strengths of our study}

- An optimisation model has not only been tested in theory but also implemented in practice to see its actual effect in the reality of a hospital. 
- It is a patient-centric system and works on a 'pull' mechanism.

- With no additional resources, waiting and cycle times have improved.

- Costs are reduced, although not measured directly.

Limitations of our study

- Require basic IT structure to collect data from all departments in the workflow.

- Data collection should happen in real-time and at the expected level to make real-time scheduling possible.

So far, a patient-centric model which looks into patient scheduling and considers patient path optimisation (a global perspective) in real-time has not been tried in an OPC in a systematic way. We have learnt from the study that this model has improved outcomes with no additional staff (human resources). This model is in real-time adjusts for the variability in actual demand.

Question 3: Does overall (global) optimisation along with real-time coordination of resources and patients improve (reduce) waiting time and cycle time?

What is known in literature?

- Resource scheduling and patient scheduling are performed separately.

- Coordination is limited to the appointments of patients only.

- OPCs are modelled as multi-agent systems (MAS), where performances are optimised ahead of time, and the results are applied by OPCs.

- Resource and patient scheduling are department-centric and are done ahead of time.

- Local optimisation leads to achieving only department-wide goals (waiting time in the individual department).

Gaps in literature

- Coordination between departments for resources and patients has not been studied.

- System status related information (real-time) sharing between departments does not exist.

- There is no global perspective in scheduling.

- Since resource and patient scheduling are not performed together, there is no coordination to match supply with demand in real-time.

Optimisation model

- A coordination mechanism to schedule resources in real-time is developed.

- Patients and resources are scheduled and rescheduled depending on the system status and demand in the OPC for minimising waiting times.

- The OPC is modelled as MAS, where resources are agents who improve their utilisation through auction bidding. 


\section{Main results}

- The real-time scheduling model reduced the average waiting time by $55.8 \%$ during simulation (from 66.3 to $29.3 \mathrm{~min}$ ) and by $51.6 \%$ during its implementation (from 66.3 to 32.1 $\min$ ) in the OPC.

- The mean cycle time reduced by $30.6 \%$ (120-83.3 min) during simulation and by $29.25 \%$ during implementation (120-84.9 min).

- Average resource utilisation improved by $14.4 \%$ during simulation and by $8.3 \%$ with imposed constraints during implementation.

- During simulation, resource utilisation improved by $12.6 \%, 13.5 \%$ and $17 \%$ for low, medium and high patient demand, respectively.

- During implementation, resource utilisation improved by $7.5 \%, 7.2 \%$ and $11.3 \%$ for low, medium and high patient demand, respectively.

- The effect of real-time coordination on resource utilisation is significant for high demand, both during simulation and implementation.

Strengths of our study

- An optimisation model has not only been tested in theory but also been implemented in practice to see its actual effect in a hospital.

- The study demonstrates that resource utilisation improves with real-time coordination.

- The optimisation model is patient-centric and operates as a "pull" system.

- Both department-wide (waiting times) and OPC-wide (cycle times) goals are achieved.

Limitations of our study

- Resources should participate actively in the coordination mechanism to work effectively and efficiently.

- Reaction time is an exogenous variable and cannot be controlled in open loop systems.

- There is a need for a basic IT structure to collect data from all departments in the workflow.

- The distance between the departments is not incorporated, which might affect waiting times.

We have learnt that demand-based resource rescheduling captures variability in demand in real-time. The global perspective improves patient flow, reduces unregulated waiting times in departments and improves cycle time across departments. The real-time coordination system improves resource utilisation. Making resource utilisation as an objective (rewarding resources) improves utilisation as well as waiting and cycle times. We have learnt that synchronisation of patients and resources in real-time improves performance in OPCs. The successful experimentation of shifting skilled resources contributes to a new approach in the optimisation methods. 
Applicability of all three models

- Can be applied to OPC systems with stochastic (walk-ins) demand and can be extended to deterministic (appointment-based) demand.

- Can be applied to single departments with subsystems and general hospitals, wherein both real-time scheduling and global optimisation will improve outcomes.

Question 4: What combination of optimisation models best improves (reduces) the waiting time and cycle time?

According to different combinations listed in Table 7.1, we describe the effects of these models on waiting times and cycle times. We compare waiting times and cycle times of all the seven models (base models as well as combination models) with the existing situation of the OPC in AEH in Figure 7.3. Additionally, we look at department-wise waiting times and summarise the results for all these models in Figure 7.4.

Model 4 is a combination of predictive resource planning and integral patient scheduling. Waiting times improved by $60 \%$ as compared to Model 1 and Model 2. Waiting times of Model 4 are less than that of Model 3 because the predictive planning provides a level of preparedness to OPCs to handle the variability caused by actual demand. Model 4 matches patient pathways to actual demand and resources to predicted demand. The variability caused by actual patient demand affects waiting and cycle times. Model 5 is not a combination of any models but is obtained by excluding patient scheduling from Model 3. We compare Model 1 , i.e., predictive resource planning that schedules resources on the basis of predicted demand, at each time-slot, with Model 5, i.e., real-time resource scheduling that reschedules resources through real-time coordination on the basis of actual demand. Model 1 improved waiting

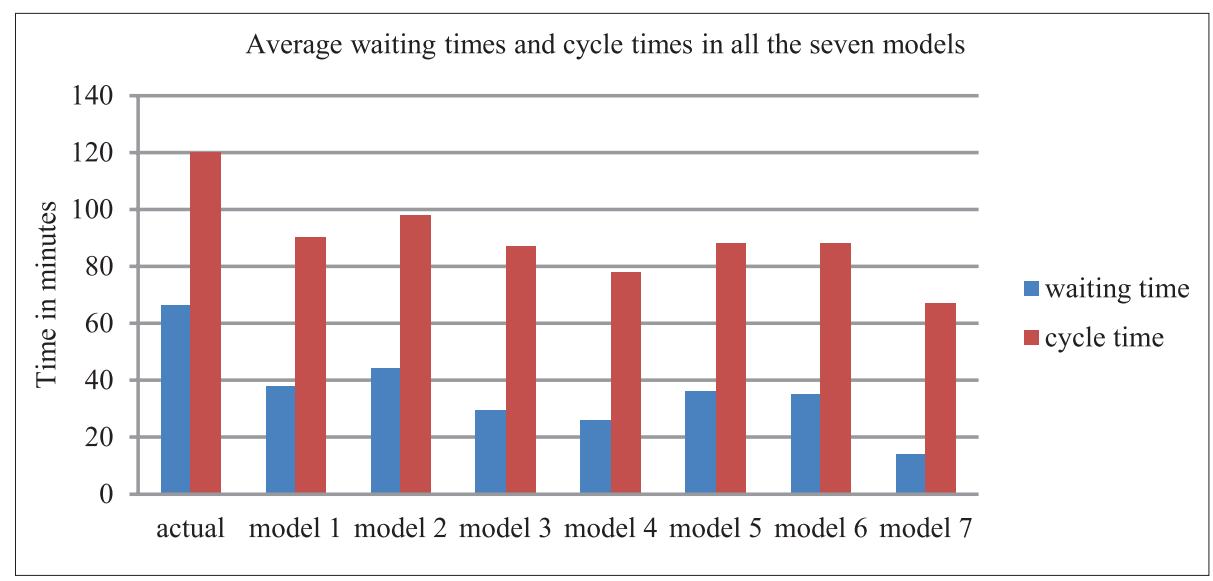

Figure 7.3: Average waiting times and cycle times in all the models 
Average waiting time in all the departments in the three basic models and their combinations

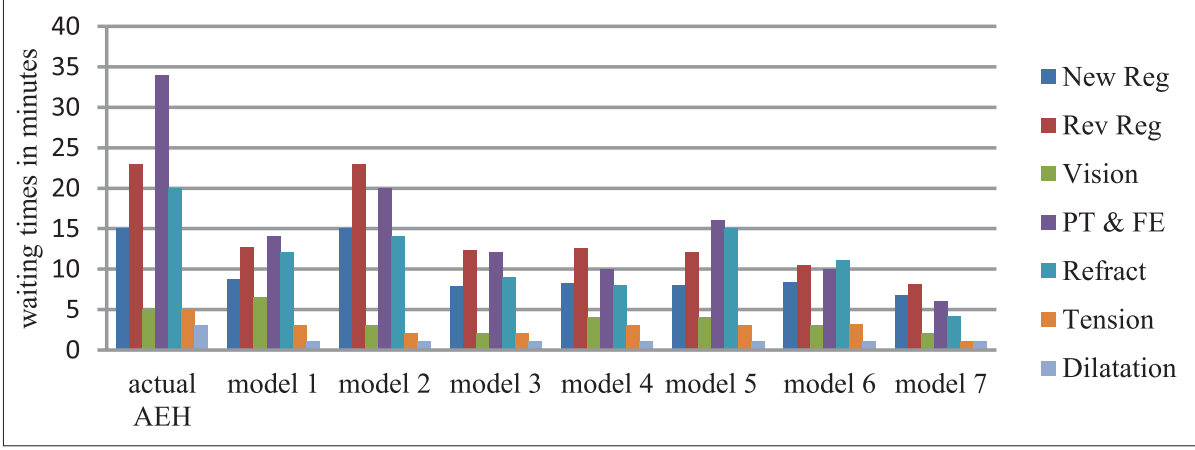

Figure 7.4: Average waiting times (minutes) in all the departments

times by 44\%; whereas, Model 5 improved it by $46 \%$ (Figure 7.3). There is little difference between the performance of Model 1 and Model 5 because of short-term demand prediction (very near to reality). Resources are aware of their schedule in Model 1; whereas, in Model 5 they are transferred when required. Here, patient scheduling is not optimised.

Further, we analyse Model 6 that is a combination of predictive resource planning and realtime resource scheduling. First, the resources are scheduled to each department on the basis of projected patient demand throughout the day, according to the schedule obtained. Further, Model 6 coordinates resources in real-time and reschedules them depending on actual demand. A level of preparedness to manage demand variability is provided by Model 1 and additional flexibility to deal with actual demand is incorporated by Model 5 . The number of transfers is less when compared to Model 3 as it is already demand-based (though predicted). Model 5 matches resources according to actual demand, but does not optimise patient scheduling. This means that it does not optimise pathways on the basis of the actual demand.

Finally, we integrate predictive resource planning, integral patient scheduling and realtime scheduling (coordination) in Model 7. We schedule resources on the basis of predictive resource planning, which matches supply to demand. Resources are aware of the plan (department and time) a day ahead. Next, the patients are scheduled on-arrival depending on actual OPC system status, in order to optimise their waiting times. Further, resources are rescheduled depending on actual demand (if variability exists). As the resource planning is demand-based, real-time rescheduling does not need more resource transfers. This combination completes the design of the operating system that includes planning, scheduling and coordination, all in real-time and in a global perspective. Latency is minimised by utilisation 
of continuous feedback from all departments for scheduling and coordination. This transforms the open loop characteristic of the OPC into a closed loop.

We have earlier discussed how a good operating system uses a combination of both push and pull in workflow optimisation. A system where care is delivered when the resources are ready is a push system, while a system where care is delivered when the patient asks for it, on the basis of system status, is a pull system. ${ }^{[1,4,23]}$ Although predictive resource planning matches supply with demand, it is a push as it is planned ahead of time and is dependent on projected demand. Both integral patient scheduling and real-time resource scheduling are pull as they are based on system status and actual demand. Model 7 makes an optimal mix of both push and pull for a good operating system.

\section{CONTRIBUTION TO THEORY}

Most of the operational modelling studies about hospitals are department-specific where planning, scheduling and control are managed locally. Scheduling in hospitals is often based on the average demand (a day, a week or a month) and ahead of time. They are not demanddriven, are not in real-time and often lack coordination.

The optimisation models in this research attempt to adapt resources and patient pathways to patient demand that varies. There is no study available on an operating system in OPCs that optimises operations in real-time with a global perspective.

Most operations in OPCs are push; whereas, pull is ideal for continuous patient flow. This research attempts to find an optimal mix of push and pul|[10] so that OPCs manage their workflows effectively and efficiently.

Predictive resource planning on the basis of forecasted demand (push) captures certain variability and uncertainty and prepares the OPCs to manage operational problems. Additionally, Takt time management has been applied to stable demand; whereas, in this study, it is applied to hospital workflow where demand is highly variable. Patient scheduling on the basis of system status (pull) allows OPCs to capture the variability and uncertainty caused by local optimisation (sequencing and scheduling). Finally, real-time resource scheduling captures the remaining variability caused by actual demand. The coordination mechanism interacts with resources in real-time and involves them actively in the optimisation. This research contributes in designing an operating system that has an intelligent and optimal combination of push and pull in OPC workflow management. 
With this research, we transform an open-loop system to a closed-loop system.

\section{CONTRIBUTION TO PRACTICE}

Since all parts of the OPC are interdependent, even if one department does not function well in a timely manner, the entire system malfunctions. The global perspective of patient flow in OPCs integrates all departments involved in the patient flow that includes individuals, groups, interactions, goals and statuses.

The operating system acts as a decision support system for analysing the future resource requirement. OPCs can utilise these optimisation models independently or in combination (together) on the basis of their working environments. The unnecessary travel of patients and resources can be eliminated. This research provides a hospital management tool that takes care of the operations management in hospitals and increases the accountability as well as utilisation of resources.

This operating system has been implemented in an OPC of a speciality eye hospital (AEH). Subsequent to the study, the hospital is continuing to use model 7 , which is a combination of all three optimisation models, and has found it very useful.

Various combinations of the three models can be applied on the basis of the situation from single OPCs, single departments to entire general hospitals. However, the extension of the models to general hospitals would require further study to adapt them in accommodating the variables specific to the particular setting.

\section{LIMITATIONS OF THE STUDY}

The study considered a non-general hospital; whereas, general hospitals differ in complexities, patient groups, processes and human interactions. The resources have to be cross-skilled to work at various departments, which is necessary for resource rescheduling to be a successful element of operations management. In this study, the resources participated proactively in rescheduling due to the working culture of $\mathrm{AEH}$, which may not always be the case in other hospitals. A greater insight would have been gained into the workflow management if the current research had included a qualitative approach (patient satisfaction, staff satisfaction, etc.). In addition, this study did not include travel time that depends on the physical layout of the OPC. Although we know that costs have been reduced, it was not measured directly. We have implemented the optimisation models only in the OPC of AEH and have not tested them in other settings. 


\section{FUTURE RESEARCH}

As part of the future research, it will be interesting to study the opportunities for developing an operating system for general hospitals with complex workflows and stochastic pathways.

Tracking of patients and resources in real-time provides accurate details about waiting time, utilisation, etc. for which the use of radio frequency identification can be explored. Along with that, the waiting time depends on travel time between departments. Therefore, we intend to explore the effects of layout optimisation on the performance of OPCs. In addition, the financial impact of the model could be examined by using cost-benefit analysis.

In conclusion, this thesis examined whether global real-time scheduling in OPCs would improve their performance and explored ways to introduce the same. The research study found that an operating system that globally schedules patients and resources in real-time improves waiting and cycle times.

\section{REFERENCES}

1. Bergeson SC, Dean JD. A systems approach to patient-centered care. JAMA 2006;296(23):2848-51.

2. Rechel B, McKee M, Haas M, Marchildon GP, Bousquet F, Blumel M, et al. Public reporting on quality, waiting times and patient experience in 11 high-income countries. Health Policy Amsterdam, Netherlands 2016;120(4):377-83.

3. Maxwell RJ. Quality assessment in health. Br Med J 1984:288:1470-2.

4. Hopp WJ, Spearman ML. Factory Physics-Foundations of Manufacturing Management. New York: Irwin, McGraw-Hill; 2001. p. 698.

5. Mintzberg H. Structure in 5's: A synthesis of the research on organization design. Manag Sci 1980;26(3):322-41.

6. Goldschmidt HM, de Vries JC, van Merode GG, Derksc JJ. A workflow management tool for laboratory medicine. Lab Autom Inf Manag 1998;33(3):183-97.

7. Van Merode F, Molema H, Goldschmidt H. GUM and six sigma approaches positioned as deterministic tools in quality target engineering. Accred Qual Assur 2004;10(1-2):32-6.

8. Noon CE, Hankins CT, Cote MJ. Understanding the impact of variation in the delivery of healthcare services. J Health Care Manag 2003;28(2):82-97.

9. Armistead C, Clark G. The "coping" capacity management strategy in service in services and the influence on quality. Int J Serv Ind Manag 1994;5(2):5-22.

10. Hopp WJ, Spearman ML. To pull or not to pull: What is the question? Manufact Serv Oper Manag 2004;6(2):133-48.

11. Ludwig M, Van Merode F, Groot W. Principal agent relationships and the efficiency of hospitals. Eur J Health Econ 2010;11(3):291-304.

12. Monreal J Jr, Valerdi R, Latt LD. A systems approach to healthcare efficiency improvement. Procedia Comput Sci 2014;28:610-8.

13. Van Merode F. A prelude of the 2004 antwerp quality conference: Targets and target values-integrating quality management and costing. Accred Qual Assur 2004;9(3):168-71.

14. Chen X, Wang LM, Ding J, Thomas N. Patient flow scheduling and capacity planning in a smart hospital environment. IEEE Access 2016;4:135-48. 
15. Harper PR. A framework for operational modelling of hospital resources. Health Care Manag Sci 2002;5(3):165-73.

16. Chaudhary B, Modi AG, Reddy K. Right to sight: A management case study on Aravind Eye Hospitals. Zenith Int J Multidiscip Res 2012;2(1):447-57.

17. Priyadarshini RG. Eye Care for All - A Case on Aravind Eye Care Systems. Coimbatore: Society for HRM (SHRM), School of Business; 2011.

18. Brilliant L, Brilliant G. Aravind: Partner and social science innovator (Innovations case discussion: Aravind eye care system). Innov Technol Governance Global 2007;2(4):50-2.

19. Molema JJ. Hospital System Design: Creating Supply Flexibility to Match Demand Variability. Maastricht, The Netherlands: Department of Health Organization, Policy and Economics, CAPHRI, FHML, Maastricht University; 2009. p. 153.

20. Aravind Eye Care System: Activity Report. Madurai; 2014-15. p. 76.

21. Prahalad CK. The Fortune at the Bottom of the Pyramid, Revised and Updated $5^{\text {th }}$ Anniversary edition: Eradicating Poverty Through Profits. New Jersey: Wharton School Publishing; 2009. p. 432.

22. Ramani KV, Mavalankar DV, Govil D. Strategic Issues and Challenges in Health Management. India: SAGE Publications; 2008. p. 227.

23. Hopp WJ, Spearman ML. Factory Physics: Foundations of Manufacturing Management. New York: Irwin, The McGraw-Hill; 1996. p. 668. 



\section{Valorisation}



This section focuses on the usefulness of the results of this thesis to researchers as well as others, such as healthcare professionals, policy makers, industry people and hospital management. This thesis includes several analyses about planning and scheduling that could be useful in minimising patient waiting times and in optimising resource utilisation. The hospital workflow optimisation services focus on capturing the existing workflow of the hospital, and an optimised solution can be simulated by applying the outcome of this study in terms of algorithms and process flows. The goal is to ensure that resources are utilised to the best and outcomes are maximised. This in turn not only leads to highly efficient systems but also reduces costs and brings in affordability.

\section{Societal Relevance}

The societal impact of this optimisation could be higher patient satisfaction due to reduced waiting times and cost effectiveness. The hospital management, one of the main stakeholders, is also benefitted for increased throughput. Overall, the society is benefitted by getting more value for the investments made in hospital infrastructure. The pressure on hospitals, due to growing patient demand, need for quality care, and limited capacity could be reduced to a great extent with intelligent planning and scheduling systems.

\section{Business and Innovation}

Research and modelling for hospital workflow optimisation have great business potential. A business model can be developed by utilising a percentage of the cost saved in sustaining the business and enabling it to grow profitably with higher volumes. Commercialisation of these models is relevant to stakeholders, as it could guide decision makers about the requirement of resources and they can identify whether to increase or reduce the number of resources. Going forward, as the model is digitised/automated, different/new clinical pathways can be discovered by using latest techniques like Artificial Intelligence and machine learning. This helps to automatically capture the best practices and replicate them in other healthcare systems.

In order to conclude, the studies presented in this thesis could be useful for patients, administrators, managers, decision makers and clinicians. It might be only a small step and there are still barriers to overcome, but this thesis might have contributed in bringing the study outcomes from operations management to various stakeholders. 

Summary 

Waiting time is an important indicator of the quality of service offered by outpatient clinics (OPCs). In spite of its growing importance, OPCs are still ill-equipped to reduce the waiting time effectively. The current applications of operations management often match patients' demand with supply. The OPC system is a push system that plans and schedules patients and resources ahead of time and is often not based on the actual demand. Additionally, OPCS are functionally organised to manage and optimise resources, patients and activities of the departments locally. In order to achieve efficiencies at the departmental level, the departments take crucial decisions with limited knowledge about other departments. Further, OPCs utilise feedback only for long term planning and scheduling.

However, in reality, OPC systems are open loop systems that have dynamic interactions with their environments. The unpredictable patient demand (due to a large number of walk-in patients, especially in Indian OPCs), unstable patient conditions and complex patient pathways result in high variability and uncertainty in OPCs. The following are the major reasons that make the OPCs' operating systems inefficient and result in prolonged waiting and cycle times, in spite of applying the existing knowledge of operating systems:

1. The planning and scheduling are performed in advance.

2. The planning and scheduling are not based on the actual patient demand.

3. The feedback is used only for future planning.

4. The entire OPC is not viewed as a single interdependent system.

Ideally, OPCs should provide services to patients when they ask for it (pull system) and to achieve this, OPCs should plan, schedule and control their resources, patients and activities in real-time with a global perspective. Therefore, rather than simply responding in an ad-hoc and individualistic manner, there is a need to systematically manage the OPC system with respect to the prevailing situation. The present thesis aims to design an operating system to minimise the waiting and the cycle times. The current thesis tests the hypothesis that " $\mathrm{A}$ hospital system with disparate subsystems cannot minimise cycle/waiting time by separately optimising subsystems, or by scheduling that does not adjust in real-time". In the present research, real-time scheduling and global perspectives are applied to each subcomponent of the operating system, namely, resource planning, patient scheduling and resource coordination. The real-time workflow optimisation uses the feedback in the same time horizon, thus reducing the latency. This transforms the OPC system from an open loop system to a closed loop system. The optimisation models and the results of all studies described in this thesis are summarised.

Chapter 1 gives an introduction to OPC systems (functional and operational structure) and explains the causes of operational problems, such as waiting and cycle times. It explains the push and the pull operating systems that are used in manufacturing industries, like Toyota Production System (TPS), albeit from the hospital context. The chapter ends with the thesis 
outline and a list of operational definitions of the terms used in the thesis. The overall research question of the present thesis "Does the overall (global) optimisation along with real-time scheduling improve the wait time/cycle time and hospital performance?" is introduced in this chapter. The research question leads to four sub-questions on resource planning, patient scheduling, resource coordination and an optimal mix of these sub-components.

Chapter 2 describes the literature survey done in the present study. This chapter examines different studies in this field and identifies the important variables required to design an operating system that improves the performance of OPCs. The chapter reviews various methods and techniques used in resource planning, scheduling of patients and resources and resource coordination. The literature review forms the basis of the present study, building on what is already known and identifying the research gaps that can be explored in this study.

Chapter 3 presents the research methodology, the design variables which were identified through the literature survey and the research strategies and design, which describes the research location (Aravind Eye Hospital (AEH), Madurai, India, one of the largest eye care providers in the world), the participants and the data collection method. The chapter ends with a presentation of the research flow.

Chapter 4 describes how a robust predictive resource planning reduces the waiting and the cycle times in an OPC. As a preliminary step, the patterns of patients' arrival and resource scheduling in AEH were studied in detail. The OPC in AEH scheduled the resources once in a month, ahead of time and on the basis of the average demand, with local control in the departments. A simulation model was built to understand the baseline functioning of the variables. In the proposed optimisation model, the predictive resource planning considered a global perspective (entire OPC) and short-term demand variability. The model employed the patient demand, the number of resources available and precedence constraints as inputs and the daily resource plan of the OPC system as output. The global perspective of resource planning was implemented through Takt time management, which is widely used in TPS.

Resource plans were obtained from the proposed optimisation model for different patient demands. The resources were accordingly scheduled both in the simulation model and in the actual model of AEH. The results confirmed that the average waiting time in the OPC was reduced by $43.4 \%$ during the simulation study and by $41.1 \%$ during its actual implementation. The reaction time is the time taken to respond to the change that is triggered by external or internal factors. Along with the demand variability and planning methods, the reaction time was also found to influence the waiting and cycle times. Therefore, the resource plans of the optimisation model were analysed with different reaction times. The present study demonstrates the matching of supplies according to short-term demand with a global perspective that enables a better planning, thereby reducing the waiting and cycle times. 
Chapter 5 examines how integral patient scheduling reduces the waiting and cycle times. Integral patient scheduling combines the concepts of a global perspective and real-time patient scheduling. The global perspective is implemented through path optimisation and the real-time patient scheduling is implemented through the actual status system of the OPC. A hybrid ant agent algorithm was developed, which considered the actual status of all departments in the OPC. The algorithm identified an optimal pathway for patients that minimised the waiting and cycle times. The proposed model was integrated into the simulation model of AEH and was implemented in their Hospital Management System. Integrated patient scheduling reduced the average waiting time in the OPC by $33 \%$ during the simulation study and by $26.5 \%$ during its actual implementation. On an average, all patients spent the same amount of time in waiting and the variability with the waiting time was observed to be reduced, as indicated by the standard deviation. Patient scheduling in real-time also depends on the IT infrastructure, which stores and retrieves knowledge of the OPC at regular and expected intervals. This study confirms that integrated patient scheduling reduced the waiting and cycle times in OPCs.

Chapter 6 describes how a real-time coordination mechanism for rescheduling resources reduces the waiting and cycle times. The individual goal of the patients is to reduce their waiting time, whereas the goal of the resources is to improve their utilisation. The goal of the OPC system is to reduce the waiting and cycle times. Therefore, the OPC system is modelled as a multi-agent system to address both individual and OPC goals. In the present study, patients are passive agents, whereas resources are active agents. The real-time scheduler schedules the patients according to the optimal pathway, depending on the actual status of all departments in the OPC. The scheduler maintains a pool to identify the available resources in the OPC and enable the rescheduling of resources. The coordination mechanism uses the Bayesian game and auction bidding methods for real-time rescheduling of resources. The resources are transferred from either the resource pool or other departments, as and when required. The coordination mechanism was analysed with different reaction times as they affect the waiting time as well. The findings of the study confirm that the real-time coordination mechanism for real-time scheduling reduced the waiting time by $55.8 \%$ during simulation and by $51.6 \%$ during actual implementation. The resource utilisation increased by $8.3 \%$ during actual implementation.

Chapter 7 discusses the main findings of each of the research questions of the present thesis. All optimisation models developed in this thesis were employed in different combinations, intended for designing the operating system. The results show that the optimal mix was the combination of predictive resource planning (push), integral patient scheduling (pull) and real-time coordination mechanism (pull). The research confirms that an OPC system with disparate subsystems cannot minimise the waiting time by separately optimising the subsystems or by scheduling that does not adjust in real-time. This chapter reflects the contribution and limitations of the present study and enlists the potential avenues for future research. 

Samenvatting 

Wachttijd is een belangrijke indicator voor de kwaliteit van de diensten aangeboden door poliklinieken. Ondanks het toenemende belang ervan zijn poliklinieken nog steeds niet goed uitgerust om wachttijden substantieel te reduceren. De huidige toepassingen van operations management in de gezondheidszorg zijn meestal gericht op het matchen van de patiëntenvraag met het aanbod. Het polikliniek-systeem is een push-systeem dat plannen en afsprakenschema's van patiënten ruim voorafgaand aan de uitvoering maakt. Deze zijn vervolgens vaak niet gebaseerd op de werkelijke vraag. Poliklinieken zijn bovendien functioneel georganiseerd en gericht op het lokaal managen en optimaliseren van middelen, patiëntenstromen en diensten van afdelingen. Om efficiënter te werken nemen afdelingen cruciale beslissingen terwijl zij maar beperkte kennis over elkaar hebben. Vaak gebruiken poliklinieken alleen feedback informatie voor lange termijn planning en niet voor de (hele) korte termijn.

In werkelijkheid zijn poliklinieken open-loop systemen die dynamisch interacteren met hun omgeving. De onvoorspelbaarheid van de patiëntenvraag (als gevolg van een groot aantal walk-in patiënten, vooral in India, patiënten met instabiele condities en complexe patiënttrajecten) leidt tot hoge variabiliteit en onzekerheid in poliklinieken. Dit leidt tot wachttijden en inefficiënt gebruik van capaciteit. Hieronder worden de belangrijkste oorzaken genoemd van het inefficiënt functioneren van poliklinieken met als gevolg lange wacht- en doorlooptijden:

1. De planning en het maken van afspraakschema's vinden ruim voor de uitvoeringsperiode plaats.

2. De planning en afsprakenschema's zijn niet gebaseerd op de werkelijke patiëntenvraag.

3. De feedback wordt alleen gebruikt voor toekomstige planning (en niet op de huidige in uitvoering zijnde planning).

4. De poliklinieken van een ziekenhuis worden niet gezien als één enkel onderling afhankelijk systeem.

In het ideale geval leveren poliklinieken hun diensten aan patiënten op het moment dat hen daar naar wordt gevraagd (pull), en om dit te kunnen doen moeten poliklinieken plannen, roosteren en monitoren in real-time met een globaal (organisatiebreed) perspectief. Daarom, in plaats van te reageren op ad hoc en individualistische wijze, zouden poliklinieken als systeem moeten reageren.

Dit promotie-onderzoek is gericht op het ontwerpen van een besturingssysteem dat wachten doorlooptijden minimaliseert. De huidige thesis toetst de volgende hypothese: "Een ziekenhuis systeem met ongelijksoortige subsystemen kan wacht-/doorlooptijden niet minimaliseren door subsystemen afzonderlijk te optimaliseren, of door niet real-time te plannen, afspraken te maken en te monitoren." In de huidige studie, worden real-time scheduling en globale optimalisatie toegepast op elk subonderdeel van het besturingssysteem. Deze subonderdelen zijn: resourceplanning, patiëntenplanning en resourcecoördinatie. Real-time workflow optimalisatie maakt gebruik van feedback informatie uit dezelfde 
uitvoeringsperiode. Dit transformeert de polikliniek van een open loopsysteem naar een gesloten kringloop.

Hoofdstuk 1 geeft een inleiding tot polikliniek systemen m.b.t. tot hun functionele en operationele structuur en de oorzaken van operationele problemen, zoals wacht- en doorlooptijden. Het bespreekt de push- en pull besturingssystemen die worden gebruikt in de industrie, zoals het TPS, in de context van het ziekenhuis. De algemene onderzoeksvraag van het huidige proefschrift 'Doet totale (globale) optimalisatie samen met real-time scheduling wacht-/ doorloop tijden in poliklinieken reduceren en prestatie maximaliseren?' wordt in dit hoofdstuk geïntroduceerd. De onderzoeksvraag leidt tot vier sub-vragen. Deze hebben betrekking op resourceplanning, patiëntenplanning, resource coördinatie en het optimaliseren van de mix van deze subonderdelen. Het hoofdstuk eindigt met een overzicht van het proefschriftoverzicht en een aantal definities van de termen die worden gebruikt.

Hoofdstuk 2 beschrijft het literatuuronderzoek uitgevoerd en de centrale variabelen die nodig zijn voor het ontwerpen van een besturingssysteem dat de prestaties van poliklinieken verbetert. Het hoofdstuk beoordeelt diverse methoden en technieken die worden gebruikt in de planning van patiënten en resources, en de coördinatie hiervan.

Hoofdstuk 3 presenteert de onderzoeksmethodologie, de variabelen en de strategieën van onderzoek en ontwerp, de methode van dataverzameling en de locatie van het onderzoek. Het ontworpen systeem is ontwikkeld voor en getest in het AEH te Madurai, India. AEH is één van de grootste aanbieders van oogzorg ter wereld.

Hoofdstuk 4 beschrijft de methodiek van resourceplanning die wacht- en doorlooptijden in een polikliniek reduceert. Als eerste stap werden de aankomstenpatronen van patiënten en de planning van resources in AEH in detail geanalyseerd. De poliklinieken in AEH plannen de middelen een maand vooruit en op basis van de gemiddelde vraag, met daarnaast lokale besturing door de afdelingen. Een simulatiemodel werd gebouwd om de huidige situatie te analyseren. In het voorgestelde model voor resource planning wordt geoptimaliseerd vanuit een globaal perspectief (de gehele polikliniek) en rekening houdend met korte termijn variabiliteit. Het optimalisatiemodel gebruikt gegevens omtrent de verwachte patiëntenvraag, het aantal beschikbare resources en de volgorde regels als input. De output van het optimalisatiemodel is de resourceplanning op dagbasis van het gehele polikliniek-systeem. Het globale perspectief van de resourceplanning werd geïmplementeerd via Takttimemanagement.

De resourceplannen werden geproduceerd op basis van verschillende vraagpatronen. Deze plannen werden vervolgens getest in een simulatiemodel en op bepaalde patiëntenstromen in het $\mathrm{AEH}$. De resultaten toonden aan dat de gemiddelde wachttijd in de polikliniek was verlaagd met $43.4 \%$ volgens de simulaties en met $41.1 \%$ in de implementatiestudie in het 
$\mathrm{AEH}$. Samen met de variabiliteit van de vraag en de planningsmethode bleken ook de reactietijden de wacht- en doorlooptijden te beïnvloeden. De reactietijd is de tijd die nodig is om te reageren op veranderingen (bijvoorbeeld de aankomst van nieuwe patiënten of het wegvallen van resources). Daarom werden de resourceplannen die door het optimalisatie-model werden geproduceerd, geanalyseerd met verschillende reactietijden. Aangetoond wordt dat het aanpassen van resources op de korte termijn, dus dicht tegen het werkelijk optreden van de patiëntenvraag, in combinatie met een globaal perspectief mogelijk is en betere plannen oplevert.

Hoofdstuk 5 onderzoekt hoe integrale patiëntplanning de wacht- en de doorlooptijden vermindert. Integrale patiëntenplanning combineert het perspectief van het geheel van poliklinieken met real-time patiëntenplanning. Dit globale perspectief wordt geïmplementeerd via padoptimalisatie en real-time scheduling door het voortdurend monitoren en reageren op de status van het poliklinieksysteem. Een hybrid ant agent algoritme werd ontwikkeld die de werkelijke status van alle afdelingen in de kliniek beschouwt. Het algoritme identificeert de optimale route voor patiënten die de wacht- en doorlooptijden minimaliseert. Het voorgestelde model werd geïntegreerd in het simulatiemodel van AEH en werd geïmplementeerd in het Ziekenhuis Managementinformatie en planningssysteem van AEH. De geïntegreerde patiënt planning vermindert de gemiddelde wachttijd in de polikliniek met $33 \%$ tijdens de studie van de simulatie en met $26,5 \%$ tijdens de eigenlijke uitvoering. De variatie in wachttijd daalde en de doorlooptijd van patiënten vertoonde daardoor ook minder variatie. Deze studie bevestigt dat de geïntegreerde patiëntenplanning de wacht- en de doorlooptijden in poliklinieken aanzienlijk reduceert. De mate waarin dit model succesvol kan worden toegepast is sterk afhankelijk van de IT-infrastructuur. Deze moet immers steeds de werkelijke situatie in de poliklinieken kunnen waarnemen en communiceren.

Hoofdstuk 6 beschrijft hoe een real-time coördinatiemechanisme voor het herplannen en realloceren van resources de wacht- en doorlooptijden vermindert. De patiënten wensen hun wachttijden te minimaliseren en de resources willen hun bezettingsgraad maximeren. Het doel van het polikliniek-systeem is het verminderen van de wacht- en doorlooptijden. Daarom is de polikliniek gemodelleerd als een multi-agent systeem om zowel individuele als polikliniek doelen te realiseren. In de huidige studie zijn patiënten passieve agenten, terwijl resources actieve agenten zijn. De real-time scheduler plant de patiënten volgens het optimale pad rekening houdend met de actuele status van alle afdelingen in de polikliniek. De scheduler beheert een overzicht van resources in de poliklinieken die beschikbaar zijn om opnieuw ingepland te worden. Het coördinatiemechanisme gebruikt Bayesiaanse spelen de veilingmethoden voor real-time resource planning en reallocatie. Het coördinatiemechanisme werd onderzocht met verschillende reactietijden omdat deze van invloed zijn op wachttijden. De bevindingen van de studie bevestigen dat de real-time coördinatiemechanismen voor real-time planning en reallocatie de wachttijd met 55,8\% reduceren tijdens 
simulatie experimenten en met 51,6\% tijdens de feitelijke implementatie. De bezettingsgraad is $8,3 \%$ gestegen tijdens de feitelijke uitvoering.

Hoofdstuk 7 bespreekt de belangrijkste bevindingen van dit onderzoek. Alle optimalisatiemodellen ontwikkeld in dit proefschrift kunnen in verschillende combinaties worden getest en geïmplementeerd. De vraag is dan welke combinatie het beste ontwerp is voor een besturingssysteem van een polikliniek. Het blijkt dat de optimale mix een combinatie betreft van voorspellende resourceplanning (push), integrale patiënt (pull-) planning en real-time coördinatie (pull). Het onderzoek bevestigt dat een polikliniek-systeem met ongelijksoortige subsystemen de wachttijden niet kan minimaliseren door subsystemen afzonderlijk te optimaliseren of door niet real-time te coördineren. 
సౌఠరంలో 



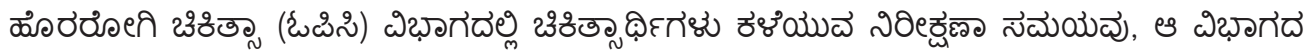

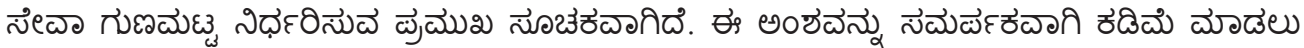

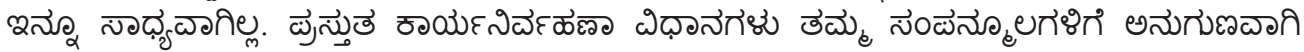

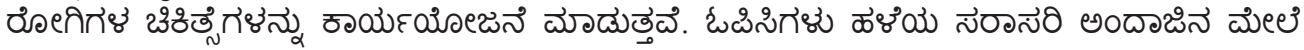

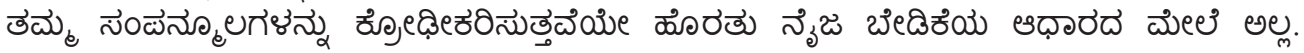

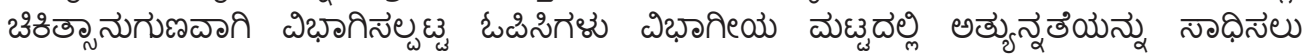

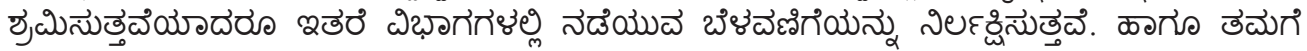

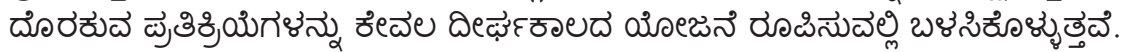

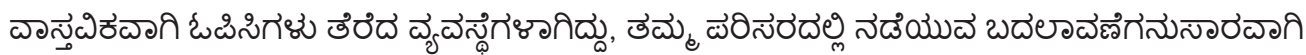

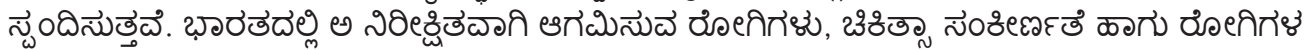

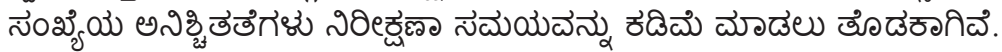

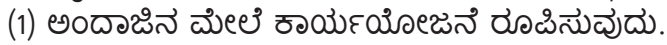

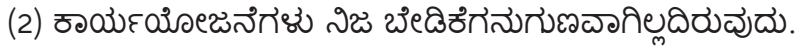

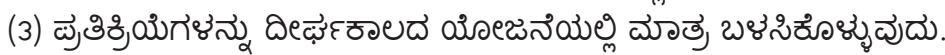

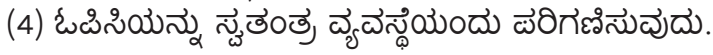

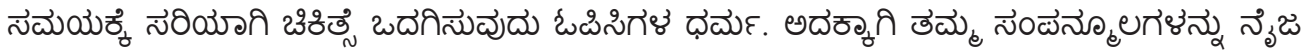

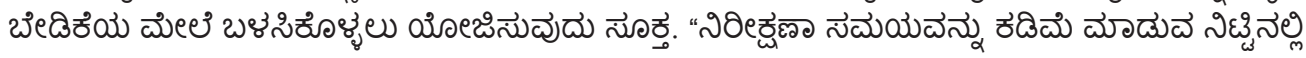

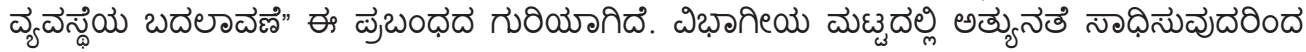

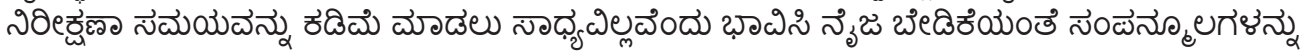

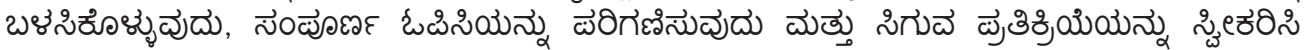

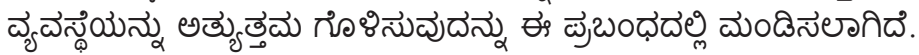

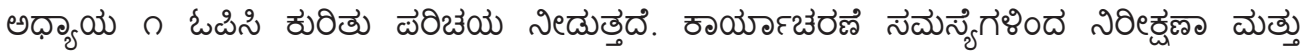

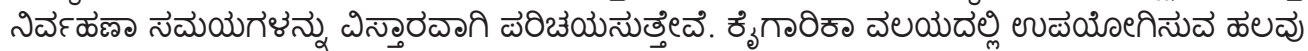

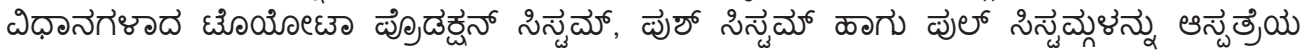

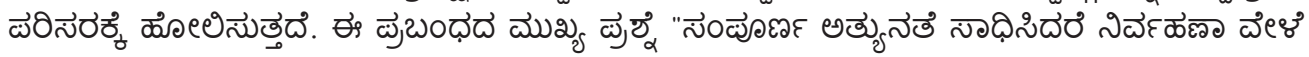

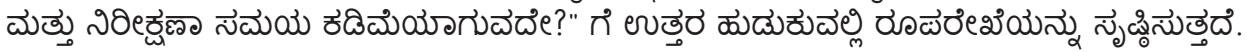

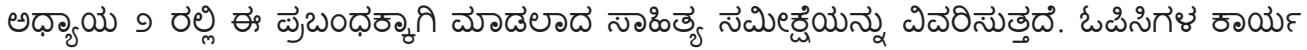

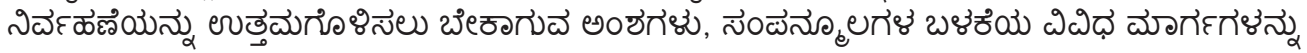
ఉరిశిలలిసుత్తదిది.

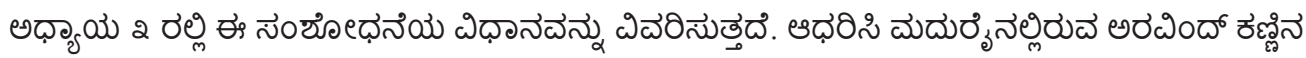

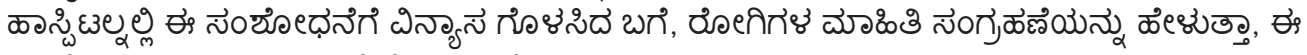

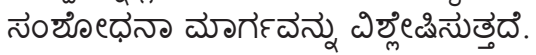

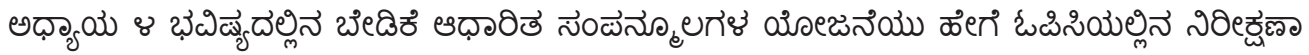

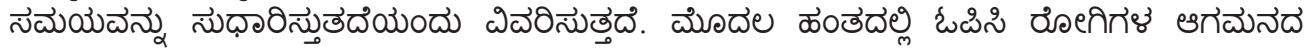




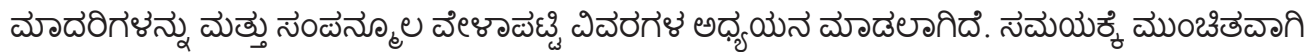

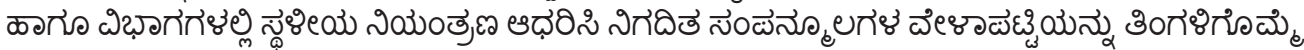

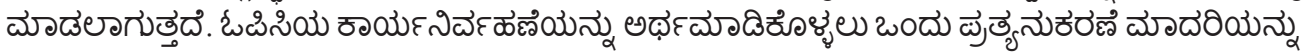

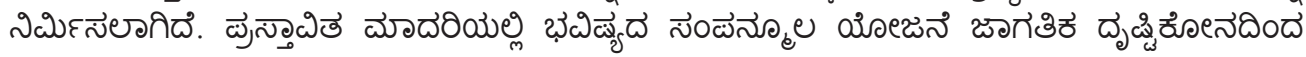
(

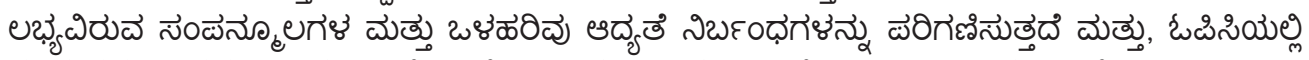

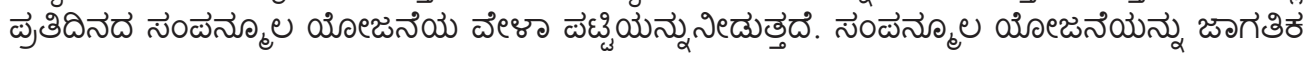

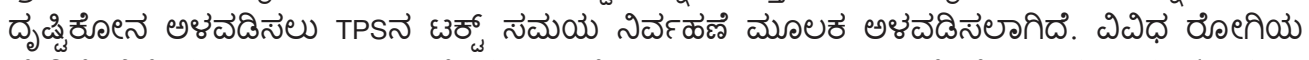

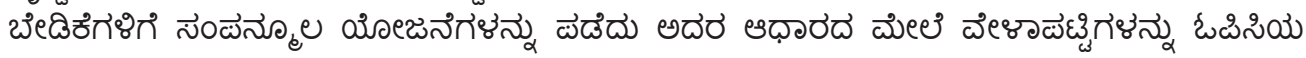

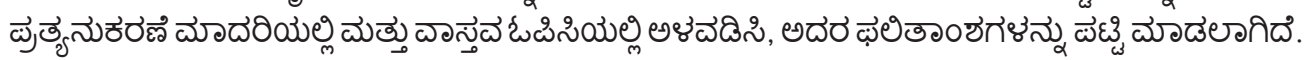

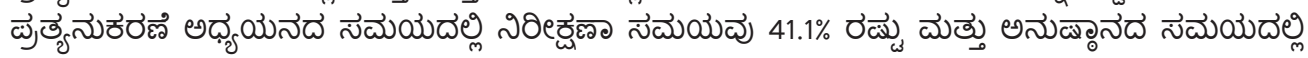

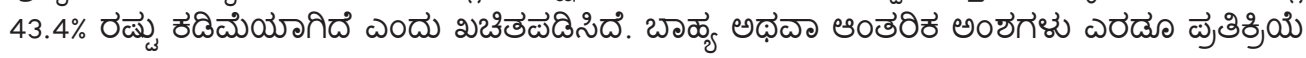

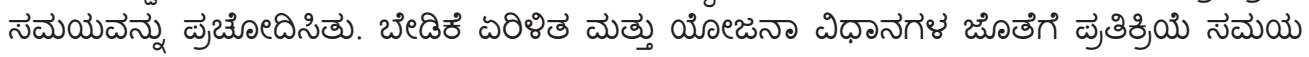

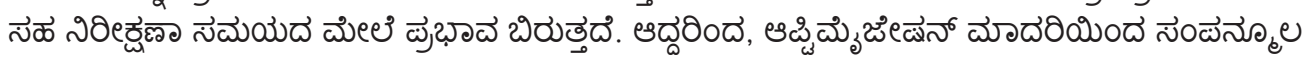

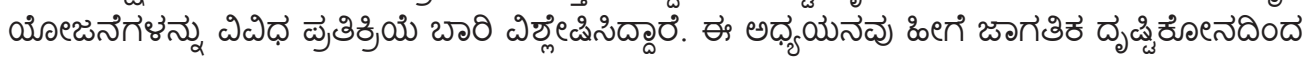

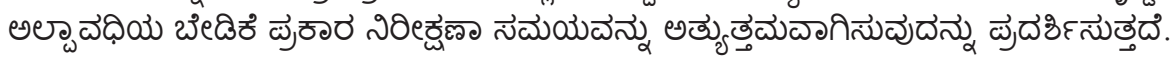

అధ్యాయ

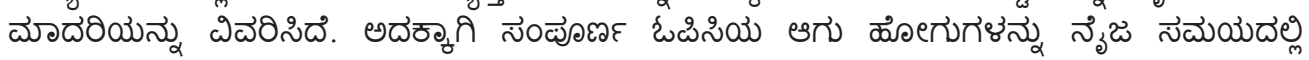

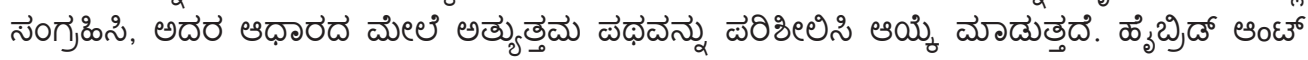

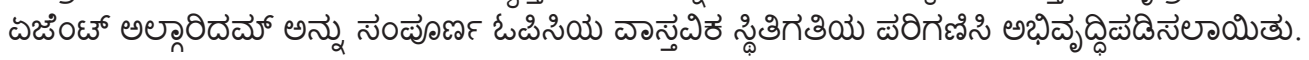

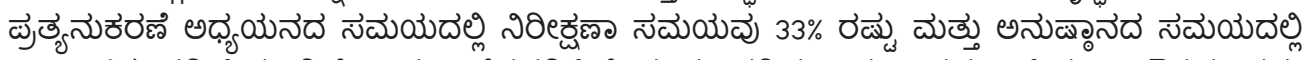

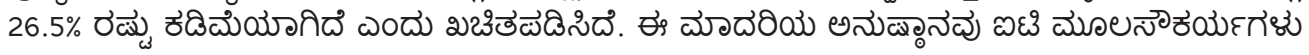

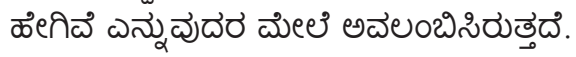

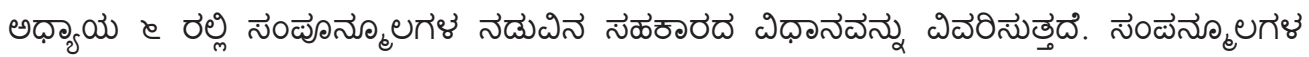

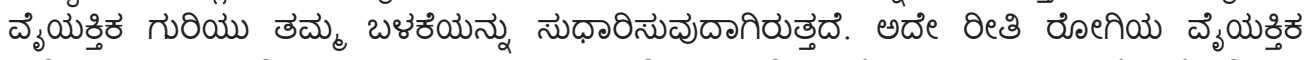

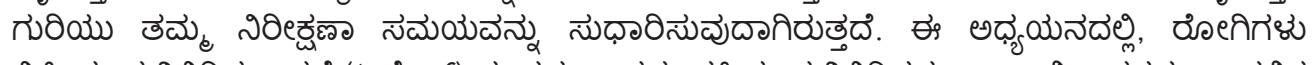

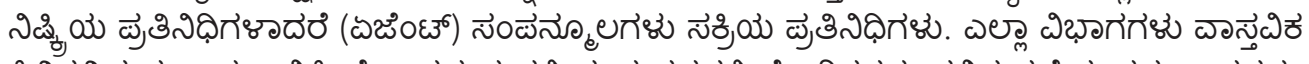

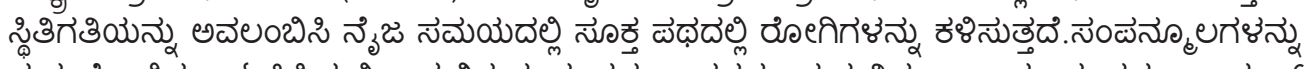

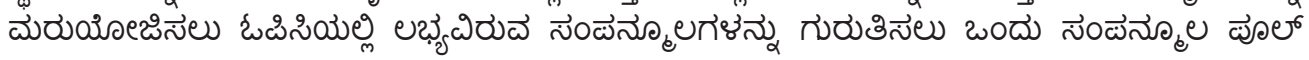

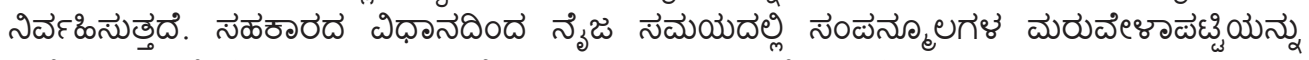

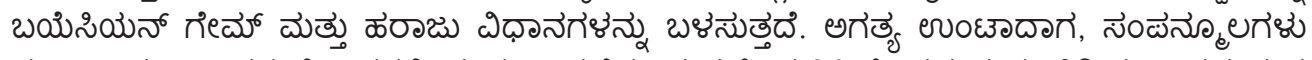

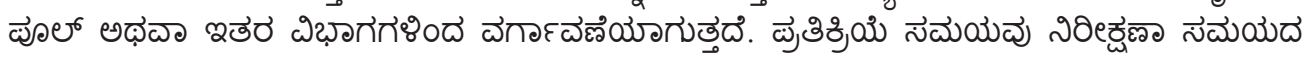

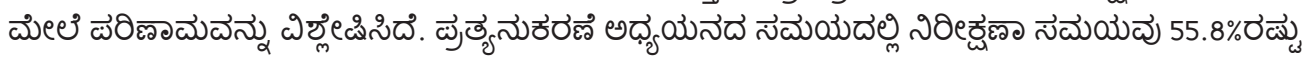

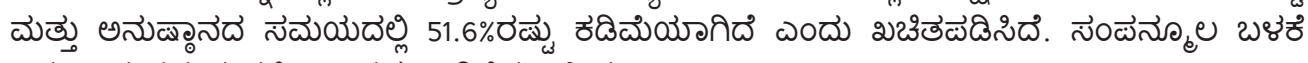

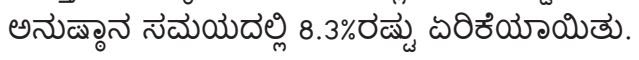

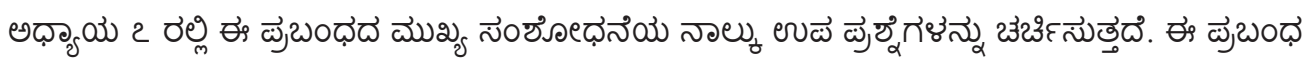

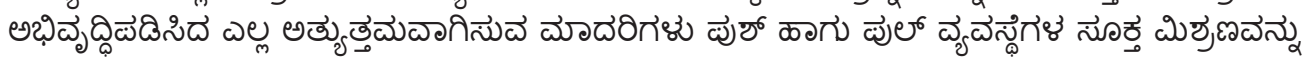




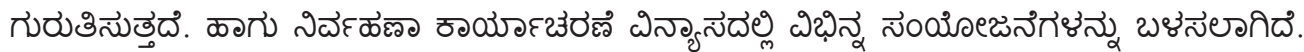

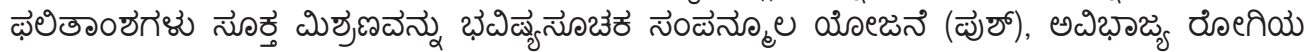

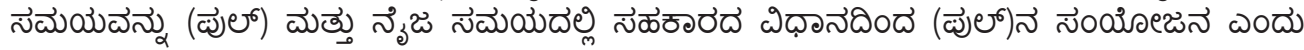

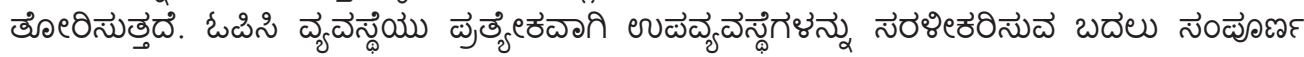

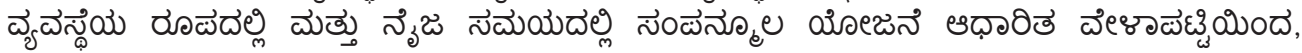

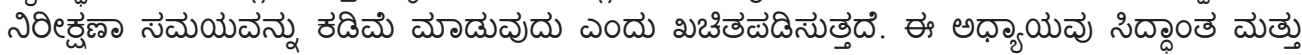

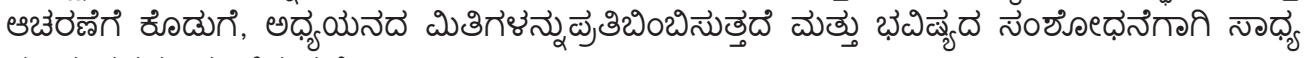

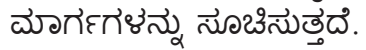



சுருக்கம் 

வெளிநோயாளர் சிகிச்சையின் தரத்தை கண்டுபிடிக்க நோயாளிகளின் காத்திருக்கும் நேரம் (OPCs) ஒரு முக்கியமான சுட்டிக்காட்டாக உள்ளது. இது முக்கியத்துவம் வாய்ந்ததாக இருந்தாலும் வெளிநோயாளர் சிகிச்சை மையங்கள் இன்னும் காத்திருக்கும் நேரத்தை திறம்பட குறைக்க முயற்சிகள் எடுக்கவில்லை. செய்முறை மேலாண்மையின் தற்போதைய பயன்பாடுகளின் வழங்கள் நோயாளிகளின் தேவைக்கு ஒத்ததாக இருக்கிறது. OPCயின் அமைப்பு ஒரு உந்துதல் அமைப்பாக உள்ளது. அதில் நோயாளிகள் அட்டவணை மற்றும் வளங்கள், பெரும்பாலும் உண்மையான தேவை அடிப்படையில் அமையவில்லை. மேலும் OPC க்களின் செயல்பாடுகள் உள்நாட்டில் நிர்வகிக்க மற்றும் வளங்களை மேம்படுத்தவும் மற்றும் நோயாளிகளையும் துறைகளின் நடவடிக்கைகளையும் மேம்படுத்த பிரயோஜனமாய் உள்ளது. துறைகளின் அளவில் செயல்திறன்களை அடைய, மற்ற துறைகளை குறித்து போதிய அறிவு இல்லாமல் முக்கியமான முடிவுகள் எடுக்கப்படுகிறது. OPC க்களை குறித்த கருத்துக்கள் நீண்ட கால திட்டமிடல் மற்றும் அட்டவணைகளை உருவாக்க பயன்படுகிறது.

எனினும், உண்மையில், ஓபிசி அமைப்புகள் ஓபன் லூப் அமைப்புகளை தங்கள் சூழலில் மாறும் திறந்த வளைய (ஓபன் லூப்) அமைப்புகள் உடையது. எதிர்பாராத நோயாளி தேவை, நிலையற்ற நோயாளி நிலைமைகள் மற்றும் சிக்கலான நோயாளி பாதைகளை குறிப்பாக இந்திய OPC காரணமாக உயர் OPC களில் அதிகமாக ஏற்படுகிறது. OPC க்கள் திறன்பட செயல்படமுடியாமைக்கு பின்வரும் இயக்க முறைமைகளில் இருக்கும் அறிவு பயன்படுத்துவதற்கு முக்கியமான காரணங்களாக கீழே கொடுக்கப்பட்டுள்ளது:

திட்டமிடல்

மற்றும்

அட்டவணை

முன்கூட்டியே ய்யப்படுகின்றன.

(2) திட்டமிடல் மற்றும் அட்டவணை உண்மையான நோயாளி வையை அடிப்படையாக கொண்டவை அல்ல.

(3) கருத்துக்களை எதிர்கால திட்டமிடுதலுக்காக மட்டுமே பயன்படுத்தப்படுகிறது.

(4) OPC க்கள் ஒன்றை ஒன்று சார்ந்திருக்கும் அமைப்பு முறையாக கவனிக்கப்படவில்லை.

வெறுமனே, OPC க்கள் நோயாளிகள் சேவைகளை உந்துதல் (மிகுதி அமைப்பு) கேட்கும் போது அதை வழங்க வேண்டும். இந்த நோக்கம் அடைய, OPCs நோயாளிகள் மற்றும் நடவடிக்கைகள் கட்டுப்படுத்த, அட்டவணை மற்றும் தங்கள் வளங்கள் உலக கண்ணோட்டம் கொண்ட உண்மையான நேரதில் திட்டமிட வேண்டும். 
எனவே, வெறுமனே ஒரு தற்காலிக மற்றும் தனிப்பட்ட முறையில் பதிலலிப்பத்தை விட, அங்கு நிலவும் சூழ்நிலையை பொறுத்து முறையாக ஓபிசி அமைப்பு நிர்வகிக்க ஓரு தேவை ஏற்படுகிறது. தற்போது ஆய்வறிக்கை காத்திருக்கும் நேரத்தில் மற்றும் சுழற்சி நேரத்தை குறைக்கும் ஒரு இயங்கும் அமைப்பை வடிவமைக்க முயற்சிக்கிறது. தற்போதைய ஆய்வறிக்கை கருதுகோள் "முற்றிலும் வேறான துணை அமைப்புகள் கொண்ட ஒரு மருத்துவமனை தனியாக துணை ஒருங்கிணைப்பதற்கும், சுழற்சி குறைக்க / காத்திருக்கும் நேரம் குறைக்க அல்லது திட்டமிடல் மூலமாக உண்மையான காத்திருக்கும் நேரத்தை குறைக்க முடியாது"

தற்போதைய ஆராய்ச்சியில், உண்மையான நேரம் திட்டமிடல் மற்றும் உலக கண்ணோட்டங்கள் இயங்க, அதாவது, வள திட்டமிடல், நோயாளி திட்டமிடல் மற்றும் வள ஒருங்கிணைப்பு ஒவ்வொரு உள் அடங்கிய பகுதியாக பயன்படுத்தப்படுகிறது. உண்மையான நேரம் முறை அதே நேரத்தில் தேர்வுமுறையை பயன்படுத்துகிறது, இதனால் மறைநிலை குறைகிறது. இந்த மூடிய கண்ணி முறையிலிருந்து திறந்த வளைய அமைப்பாக ஓபிசி அமைப்பு உருமாறுகிறது. இப்படியாக தேர்வுமுறை மாதிரிகள் மற்றும் அனைத்து ஆய்வுகளின் முடிவுகள் சுருக்கமாக விவரிக்கபட்டுள்ளன.

அத்திகாரம் 1 OPC அமைப்புகள் (செயல்பாட்டு மற்றும் செயல்பாட்டு அமைப்பு) காத்திருக்கும் நேரம் மற்றும் சுழற்சி நேரம் போன்ற செயல்பாட்டு சிக்கல்களையும், காரணங்களை குறித்து அறிமுகப்படுத்து -கிறது. அது என்னவெனில் டொயோட்டா உற்பத்தி இயக்கம் (TPS) போன்ற உற்பத்தித் தொழில்களில் பயன்படுத்தப்படும் இழு மற்றும் தள்ளு இயக்க முறைகளைபோல மருத்துவமனைகளிலும் செயல்படுகின்றன. ஆய்வறிக்கை அவுட்லைன் மற்றும் ஆய்வறிக்கை -யில் பயன்படுத்தப்படும் சொற்கள் செயல்பாட்டு வரைமுறைகளுடன் அதிகாரம் முடிவடைகிறது. தற்போது ஆய்வறிக்கை ஒட்டுமொத்த ஆராய்ச்சி கேள்வி 'ரியல் (Real) நேரம் திட்டமிடல் சேர்த்து ஒட்டுமொத்த (உலக) தேர்வுமுறை காத்திருப்பு நேரம் / சுழற்சி நேரத்தையும் மருத்துவமனையில் செயல்திறனை மேம்படுத்த வழிமுறைகளையும் இந்த அதிகாரத்தில் அறிமுகப்படுத்துகிறது. வளங்கள் திட்டமிடல், நோயாளி அட்டவணை, வளங்கள் ஒருங்கிணைப்பு மற்றும் உகந்த கலவையின் உல்லடங்கிய பகுதி ஆராய்ச்சி கேள்வியின் நான்கு துணை கேள்விகளாகும்.

அதிகாரம் 2, தற்போதைய ஆய்வில் இலக்கிய ஆய்வை விவரிக்கிறது. இந்த அதிகாரம் இந்த துறையில் உள்ள பல்வேறு 
ஆய்வுகளை பரீட்சித்து ஒரு இயக்க முறைமையை வடிவமைத்து தேவையான முக்கியமான காரணிகள் அடையாளம் கொண்டு OPC களின் செயல்திறனை அதிகரிக்கிறது. வளம் திட்டமிடல், நோயாளிகள் அட்டவணை மற்றும் வளங்கள் திட்டமிடல், மற்றும் வள ஒருங்கிணைப்பு பயன்படுத்தப்படும் பல்வேறு முறைகள் மற்றும் நுட்பங்களை மதிப்பிடுகிறது இந்த அதிகாரம். தற்போதைய ஆய்வு, ஏற்கனவே அறியப்பட்ட ஆராய்ச்சியின் மேல்கட்டி, ஆராய்ச்சி அடையாளங்களை கண்டுபிடித்து, தற்போதைய ஆய்வின் அடிப்படையாக விளங்குகிறது இந்த இலக்கிய விமர்சனம்.

மூன்றாம் அதிகாரம் இலக்கிய சர்வே மூலம் அடையாளம் காணப்பட்ட ஆராய்ச்சி முறை, வடிவமைப்பு மாறிகள், மற்றும் ஆராய்ச்சி உத்திகள் மற்றும் வடிவமைப்பு, பங்கேற்பாளர்கள் மற்றும் தரவு சேகரிப்பு முறையை விவரிக்கிறது. ஆராய்ச்சி இடம் (இந்தியாவில் மதுரையில் உள்ள அரவிந்த் கண் மருத்துவமனை (AEH) உலகில் பெரிய கண் பாதுகாப்பு வழங்குநர்கள்). அதிகாரம் ஆராய்ச்சி ஓட்டம் வழங்கலுடன் முடிவடைகிறது.

அதிகாரம் 4 ஒரு வலுவான முன்னறிவிக்கும் வள திட்டமிடல், வெளிநோயாளர் மருத்துவமனையில் காத்திருக்கும் நேரத்தை மற்றும் சுழற்சி நேரத்தை குறைக்கும் என்பதை விவரிக்கிறது. ஒரு பூர்வாங்க கட்டமாக, AEH நோயாளிகளின் 'வருகையை வடிவங்கள் மற்றும் வள அட்டவணைகளை விரிவாக ஆய்வு செய்யப்பட்டது. AEH உள்ள OPC வளங்கள் மாதத்திற்கு ஒரு முறையாவது, குறிப்பிட்ட நேரத்துக்கு முன்பாக சராசரி தேவை அடிப்படையில், உள் துறைகள் கட்டுப்பாடு திட்டமிடப்பட்டுள்ளது. ஒரு உருவகப்படுத்துதல் மாதிரி மாறிகளின் அடிக்கோட்டு செயல்பாட்டை புரிந்து கொள்ள கட்டப்பட்டது. முன்மொழியப்பட்ட தேர்வுமுறை மாதிரியில், முன்னறிவிக்கும் வள திட்டமிடல் உலக கண்ணோட்டம் (முழு OPC) மற்றும் ஒரு குறுகிய கால தேவை மாறுதன்மையே கருதப்படுகிறது. இந்த மாதிரி, நோயாளி தேவை, கிடைக்கும் வளங்கலளின் எண்ணிக்கை, மற்றும் உள்ளீடுகள் முன்னுரிமை கட்டுப்பாடுகளையும் மற்றும் வெளியீடு OPCஅமைப்பு தினசரி வள திட்டம் வேலையும் செயல்படுத்துகிறது. வள திட்டமிடலை உலக முன்னோக்கு பரவலாக பயன்படுத்தப்படும் TPS ய யன்படுத்தப்படும் TAKT நேரம் மோண்மை, மூலம் செயல்படுத்தப்பட்டது.

வள திட்டங்கள் வெவ்வேறு நோயாளி கோரிக்கைகள் ன்மொழியப்பட்ட தேர்வுமுறை மாதிரியில் இருந்து பெறப்படுகின்றன. வளங்கள் அதன்படி உருவகப்படுத்துதல் மாதிரி மற்றும் AEH உண்மையான 
மாதிரி இரண்டிலும் திட்டமிடப்பட்டது. முடிவுகள் OPC சராசரி காத்திருக்கும் நேரத்தில் உருவகப்படுத்துதல் ஆய்வின் போது, 43.4\% அகா குறைக்கப்பட்டது என்றும், மற்றும் உண்மையான செயல்படுத்தப்பட்ட போது $41.1 \%$ ஆக குறைந்தது. எதிர்வினை நேரம் உள் காரணி அல்லது வெளி காரணிகளால் தூண்டப்படுகிறது என்றும் மாற்றம் பதிலளிக்க எடுத்துக்கொள்ளும் நேரம் ஆகும். தேவை மாறுதன்மை மற்றும் திட்டமிடல் முறைகளுக்கு இணைந்து, எதிர்வினை நேரம் காத்திருக்கும் நேரத்தில் மற்றும் சுழற்சி நேரத்தில் தாக்கம் இருப்பதாகக் கண்டுபிடிக்கப்பட்டது. எனவே, தேர்வுமுறை மாதிரி வள திட்டங்கள் வெவ்வேறு எதிர்வினை முறையில் இணைந்து பகுத்தாய்வு செய்யப்பட்டது. தற்போதைய ஆய்வு ஒரு சிறந்த திட்டமிடலை செயல்படுத்துகிறது என்று குறுகிய கால தேவைகளுக்கு ஏற்ப, ஒரு உலக முன்னோக்கு பொருட்கள் பொருந்தும் என நிரூபித்ததால் காத்திருக்கும் நேரம் மற்றும் சுழற்சி நேரம் குறைந்தது.

OPC யில் உள்ள அனைத்து துறைகள் உண்மையான நிலையை கருதி கலப்பின ANT முகவர் வழிமுறை உருவாக்கப்பட்டது. காத்திருக்கும் நேரம் மற்றும் சுழற்சி நேரம் குறைத்த நோயாளிகள் ஒரு வழிமுறைமூ -லமாக உகந்த வழித்தடம் அடையாளம் காணப்பட்டது. உத்தேச மாதிரியை AEH உருவகப்படுத்தல் மாதிரி ஒருங்கிணைக்கப்பட்ட AEH மருத்துவமனையில் மேலாண்மை அமைப்பில் செயல்படுத்தப்பட்டது.

ஒருங்கிணைந்த நோயாளி திட்டமிடல் உருவகப்படுத்துதல் ஆய்வின் போது 33\% OPC உள்ள சராசரி காத்திருக்கும் நேரம் குறைக்கப்பட்டது, மற்றும் அதன் உண்மையான செயல்படுத்தின போது $26.5 \%$ ஆக குறைந்தது. சராசரியாக, அனைத்து நோயாளிகள் காத்திருக்கும் நேரம் அதே அளவாய் இருந்தது, மற்றும் திட்ட விலக்கல் சுட்டிக்காட்டிய காத்திருக்கும் நேரம் மாறுபடும் குறைக்கப்பட வேண்டும் என அனுசரிக்கப்பட்டது. வழக்கமான மற்றும் எதிர்பார்க்கப்படுகிற இடைவெளியில் OPC யில் இருந்து அறிவு பெற்று நோயாளி காத்திருக்கும் உண்மையான நேரத்தில் திட்டமிடல் IT ள்கட்டமையை பொறுத்தது. இந்த ஆய்வு ஒருங்கிணைந்த நோயாளி திட்டமிடல் காத்திருக்கும் நேரம் மற்றும் OPCs சுழற்சி நேரம் குறைக்கப்பட்டது என்பதை உறுதிப்படுத்துகிறது.

அதிகாரம் 6 காத்திருக்கும் நேரத்தில் மற்றும் சுழற்சி முறை மீண்டும் திட்டமிடல் (rescheduling) வளங்கள் எப்படி உண்மையான நேரம் ஒருங்கிணைப்பு பொறிமுறையை குறைக்கிறது என்பதை 
விவரிக்கிறது. நோயாளிகள் தனிப்பட்ட இலக்கு, தங்கள் காத்திருக்கும் நேரம் குறைக்க வேண்டும். அதேசமயம், வளங்களிலன் இலக்கு அவர்கள் பயன்பாடு மேம்படுத்தவேண்டும். OPC அமைப்பின் இலக்கு காத்திருக்கும் நேரம் மற்றும் சுழற்சி நேரம் குறைக்க வேண்டும் என்பதே. எனவே, OPC தனி மற்றும் OPC யின் இலக்குகளை நிவர்த்தி செய்ய ஒரு பல-ஏஜெண்ட் அமைப்பு மாதிரியாக உள்ளது.

தற்போதைய ஆய்வில், ோாயாளிகள் செயலற்ற காரணிகளாக உள்ளன. அதேசமயம் வளங்கள் செயலில் முகவர்கள். நோயாளிகளின் உண்மையான நேரம் திட்டமிடுதலின் கால அட்டவணைகளின் உகந்த பாதை, OPC யில் உள்ள அனைத்து துறைகளின் உண்மையான நிலையை பொறுத்தது. OPC யின் திட்டமிடுதலின் குளம் வளங்களை அடையாளம் மற்றும் வளங்கள் அட்டவணையை மாற்றி செயல்படுத்த பராமரிக்கிறது.

ஒருங்கிணைப்பு பொறிமுறை உண்மையான நரம் வள அட்டவணையை மாற்றி அமைக்கவும் Bayesian Game மற்றும் auction bidding முறைகளை பயன்படுத்துகிறது. தேவைப்படும்போது வளங்கள் அல்லது மற்ற துறைகளின் வளங்களிள் இருந்து மாற்றப்படும். எதிர்வினை நேரம் கூட காத்திருக்கும் நேரத்தை பாதிக்கும் என்பதால் ஒருங்கிணைப்பு பொறிமுறையை வெவ்வேறு எதிர்வினை முறை கொண்டு ஆராயப்பட்டது. இந்த ஆய்வின் கண்டுபிடிப்புகள் உண்மையான நேரம் திட்டமிடல் உண்மையான நேரம் ஒருங்கிணைப்பு பொறிமுறையை உண்மையான செயல்படுத்தலின் போதும் உருவகப்படுத்துதலின் போது 51.6\% மற்றும் காத்திருக்கும் நேரம் மூலம் $55.8 \%$ ஆக குறைக்கப்பட்டது என்பது உறுதி. வளங்களை உண்மையாக செயல்படுத்த போது 8.3\% ஆக அதிகரித்துள்ளது.

அதிகாரம் 7 தற்போதைய ஆய்வறிக்கை ஆராய்ச்சி கேள்விகளில் ஓவ்வொரு முக்கிய கண்டுபிடிப்புகளை விவாதிக்கிறது. இ இந்த ஆய்வறிக்கை அபிவிருத்தியடைந்த அனைத்து தேர்வுமுறை மாதிரிகளின் நோக்கம் பல்வேறு ேர்க்கைகள் இயங்கும்படி வடிவமைக்கப்பட்டது. வள திட்டமிடல் (push), ஒருங்கிணைந்த நோயாளி திட்டமிடல் (pull) மற்றும் உண்மையான நேரத்தில் ஒருங்கிணைப்பு பொறிமுறையை (pull) இணைந்த உகந்த கலவை முடிவாக காட்டுகிறது. OPC அமைப்பு தனியாக ஒருங்கிணைப்பதற்கும் துணை அமைப்புகள் மூலம் அல்லது உண்மையான நேரத்தில் திட்டமிடல் மூலமாக காத்திருக்கும் நேரம் குறைக்க முடியாது என்பதை ஆராய்ச்சி முற்றிலும் உறுதிப்படுத்துகிறது. இந்த அதிகாரம் 
தற்போதைய ஆய்வு பங்களிப்பு மற்றும் வரம்புகளைப் பிரதிபலிக்கிறது மாற்றுமல்லாமல் எதிர்கால ஆராய்ச்சிகளுக்கு வழிவகுகிறது. 
Acknowledgements 

One of the most beautiful gifts in the world is the gift of encouragement. When someone encourages you, that person helps you over a threshold you might otherwise never have crossed on your own.

John O’ Donohue

Completion of this thesis could only be possible with the guidance of my supervisors, help and support of my family members and friends. The journey of research has been a great learning experience for me, both professionally and personally.

Foremost, I would like to express my sincere gratitude to my supervisor Prof. Frits van Merode, $\mathrm{ClO}$ Executive Board, Professor of Operations Management and Logistics in Healthcare, Maastricht UMC+, Maastricht University, The Netherlands, for his continuous support, knowledgeable discourse, patience, motivation, enthusiasm, and cooperation during my PhD. His guidance helped me throughout my research and writing of this thesis. I could not have expected to have a better supervisor and mentor than him for my PhD. I greatly appreciate his confidence in me during the writing of the thesis, welcomed my questions readily, and cultivated the habit of viewing the issues on multiple prospects. His vision, critical thinking and guidance abilities are unique. I will remain indebted for changing the course of my life.

Words are not enough to thank my co-supervisor Dr. Shyam Vasudeva Rao, Technical Director, Maastricht University Education and Research Centre, The Netherlands. I take this opportunity to acknowledge the guidance and continuous support of him throughout this research. He encouraged me in difficult times and proposed solutions to reach here. I am fortunate to have learned a lot from him and his vast research experience. I thank him for providing an opportunity that has broadened my horizon.

I am grateful to Dr. S. Aravind, Administrator, Aravind Eye Hospitals, Madurai and Director Projects, Aravind Eye Care System, without his valuable guidance, support, and encouragement, this research would not have been possible. I sincerely thank him for providing me valuable insight in the development and implementation of the research.

I thank the evaluation committee for showing interest in my work and taking the time to review my thesis.

I wish to express my profound thanks to Dr. K.N. Balasubramanya Murthy, Vice Chancellor, PES University for providing administrative support, space and day guide support throughout the course of the PhD. I also thank Dr. A. Srinivas, Professor and Dean, School of Engineering, Dayanand Sagar University, Bangalore, India, for his kind guidance and support to make this 
research success. I thank Dr. Tim Poston, Chief Scientist at Sankhya Sutra Labs Private Limited for his guidance during the writing of my thesis.

My sincere thanks also go to the IT department, administrative managers, and the staff of Aravind Eye Hospital, Madurai, for assisting me with the study and implementation period of this research. Special thanks to the people of Aravind Harmony Guest House for making me feel at home.

I thank my parents for their infinite patience, unconditional love, believing in my dreams and being with me when I needed the most. I especially thank my mother who is a source of inspiration. I thank my husband for his continuous encouragement and understanding for the completion of my PhD. I thank my son Rajat for his love and cooperation. I thank my family and friends for their continuous support during my research. 
About the Author 

Jyoti Raghavendra Munavalli was born in Koppal, Karnataka, India on 29 Sept 1979. After finishing her Secondary education in 1995, she studied Science as major at a pre-university college in India (Gadag, Karnataka). In 2001, she graduated with Bachelor of Engineering in Electronics and Communication from Karnataka University, Dharwad. In 2009, she completed her Masters in Technology with specialisation in Digital Electronics and Communication from Vishveswaraya Technological University, Belgaum. Her project on pattern recognition using artificial neural networks identified and restored the actual data from corrupted ones with high accuracy. She worked as a faculty in various Engineering colleges for 6 years and supervised various undergraduate and postgraduate student projects. She started her PhD as a research associate with the Faculty of Health, Medicine, and Life Sciences at Maastricht University Medical Centre, The Netherlands, and PES Institute of Technology, Bangalore, India, in 2010, under the guidance of Prof. Frits van Merode and Dr. Shyam Vasudeva Rao. Her research focussed on real-time operations management in outpatient clinics. Jyoti worked closely with Aravind Eye Hospital System on workflow optimisation. She has interests in Multi-agent system modelling, real-time tracking and scheduling, artificial intelligence and self-correcting networks. 

